\title{
Volatile Organic Compound Investigation Results, 300 Area, Hanford Site, Washington
}

\author{
RE Peterson \\ BA Williams \\ RM Smith
}

July 2008 


\title{
Volatile Organic Compounds Investigation Results, 300 Area, Hanford Site, Washington
}

\author{
RE Peterson \\ BA Williams $^{(a)}$ \\ RM Smith
}

July 2008

Prepared for

the U.S. Department of Energy

under Contract DE-AC05-76RL01830

Pacific Northwest National Laboratory

Richland, Washington 99352

(a) Fluor Hanford, Inc., Richland, Washington 



\section{Acknowledgments}

The following individuals were responsible for the content and authorship of the main subsections in this report: R. E. Peterson (introduction, background, laboratory results, and shoreline data); B. A. Williams (drilling results and hydrogeology); and R. M. Smith (pathways discussion).

The authors benefited from careful and thoughtful content reviews by B. N. Bjornstad, M. J. Truex, and K. M. Thompson. J. A. Horner and B. N. Bjornstad prepared the detailed lithologic descriptions of sediment encountered during drilling and subsequent core sample analysis. D. C. Lanigan produced the detailed cross sections and composite borehole logs that are so helpful in telling the story. P. D. Thorne and J. A. Horner produced the updated structure contour map graphics that illustrate the distribution of the VOC contamination in the Ringold Formation. A. J. Currie provided final technical editing support, and M. J. Parker, S. A. Larsen, K. R. Neiderhiser, and L. M. Andor all contributed to the final layout and assembly of this report. 



\section{Executive Summary}

Unexpectedly high concentrations of volatile organic compounds (VOCs) were discovered while drilling in the unconfined aquifer beneath the 300 Area of the Hanford Site during 2006. The discovery involved an interval of sediment within the unconfined aquifer that is not routinely monitored by groundwater sampling. Although VOC contamination in the unconfined aquifer has been identified previously and monitored, the concentrations of newly discovered contamination are much higher, with some new results significantly above the drinking water standards. The primary contaminant is trichloroethene, with lesser amounts of tetrachloroethene. Both chemicals were used extensively as degreasing agents during the fuels fabrication process. A microbiological degradation product of these chemicals, cis-1,2-dichloroethene, also was detected.

To further define the nature and extent of this contamination, additional characterization drilling was undertaken during 2007. Four locations were drilled to supplement the information obtained at four locations drilled during the earlier investigation in 2006. The results of the combined drilling indicate that the newly discovered contamination is limited to an interval within the Ringold Formation that contains relatively finer-grained sediment than is found in other portions of the unconfined aquifer. The lateral extent of this contamination appears to be the area immediately east and south of the former South Process Pond. Samples collected from the finer-grained sediment at locations along the shoreline confirm the presence of the contamination near the groundwater-river interface. Contamination was not detected in river water that flows over the area where the river channel potentially incises the finer-grained interval of aquifer sediment.

The source for this contamination is not readily apparent. A search of historical documents and the Hanford Waste Information Data System did not provide definitive clues as to specific waste disposal operations or spills that might have resulted in groundwater contamination in this sediment, although several relatively small accidental releases of VOCs have occurred in the past in the northern portion of the 300 Area. It is likely that large quantities of degreasing solutions were disposed to the North and South process ponds during the 1950s and 1960s. The infiltration of these discharges through the vadose zone was probably sufficient to cause widespread contamination of the underlying aquifer, and to depths that included the finer-grained material where contamination currently remains because of the low permeability associated with this sediment. Evidence for the earlier widespread contamination in the upper portion of the unconfined aquifer has been removed because of rapid groundwater movement through the much more transmissive sediment. Investigations to date have revealed no evidence to suggest that a dense, nonaqueous phase liquid remains undetected in the subsurface.

Potential pathways for contamination to migrate from this finer-grained sediment include groundwater movement through the interval to offshore locations in the Columbia River channel, dispersion out of the finer-grained interval into the overlying transmissive sediment (again, with transport to the riverbed), and potential future withdrawal via water supply wells. However, the rate of withdrawal from a water supply well would be dominated by groundwater from the relatively uncontaminated saturated Hanford formation sediment, and any additional contaminant contribution caused by release from the finer-grained interval would likely have little impact on the quality of groundwater withdrawn. 



\section{Acronyms and Abbreviations}

$\begin{array}{ll}\mathrm{CCL}_{4} & \text { carbon tetrachloride } \\ \text { DCE } & \text { 1,2-dichloroethene (cis-1,2-DCE or 1,2-DCE total) } \\ \text { DNAPL } & \text { dense, nonaqueous phase liquid } \\ \text { EPA } & \text { U.S. Environmental Protection Agency } \\ \text { HEIS } & \text { Hanford Environmental Information System } \\ \text { HWIS } & \text { Hanford Well Information System } \\ \text { LFI } & \text { limited field investigation } \\ \text { MCL } & \text { maximum contaminant level } \\ \text { MDL } & \text { method detection limit } \\ \text { PCE } & \text { tetrachloroethene } \\ \text { RACS } & \text { Remedial Action and Closure Science } \\ \text { TCE } & \text { trichloroethene } \\ \text { VC } & \text { vinyl chloride } \\ \text { VOC } & \text { volatile organic compound }\end{array}$





\section{Contents}

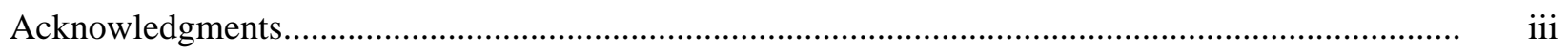

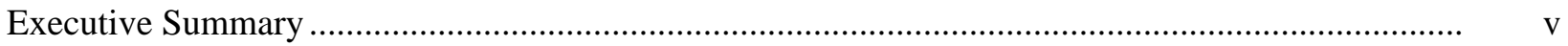

Acronyms and Abbreviations ..................................................................................................... vii

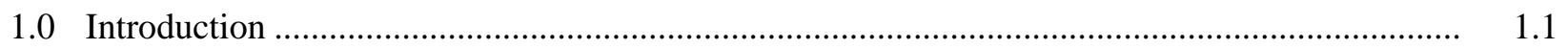

1.1 Scope of Volatile Organic Compound Investigation...................................................... 1.1

1.2 Environmental Chemistry of Trichloroethene, Tetrachloroethene, and

1.3 Drilling and Characterization Methodology............................................................ 1.6

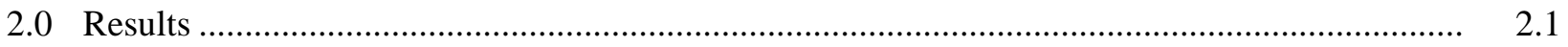

2.1 Contaminant Observations During LFI and VOC Characterization Drilling ...................... 2.1

2.2 New Hydrogeologic Information from VOC Investigation Boreholes .............................. 2.9

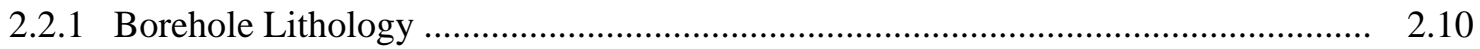

2.2.2 Depth-Discrete Aquifer Hydraulic Testing Results .............................................. 2.12

2.2.3 Spectral Gamma and Neutron Moisture Geophysical Logging ............................. 2.17

2.2.4 Composite Borehole Logs ............................................................................ 2.17

2.2.5 Subsurface Characterization............................................................................... 2.17

2.3 VOC Concentrations at Shoreline Aquifer Tube Sites.................................................... 2.20

2.4 VOC Contamination in the Columbia River Environment .............................................. 2.24

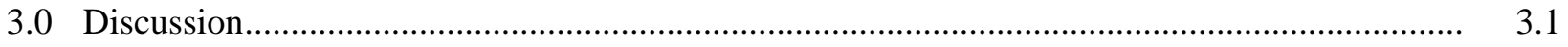

3.1 Potential Sources for TCE, PCE, and DCE Observed in Drilling Samples ....................... 3.1

3.2 Environmental Pathways Associated with VOC Contamination ....................................... 3.6

3.2.1 Pathways to Exposure ........................................................................................... 3.6

3.2.2 Aquifer Pathway Evaluation .............................................................................. 3.6

3.3 VOC Investigation and cis-DCE at Well 399-1-16B ................................................... 3.8

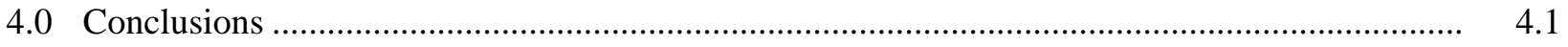

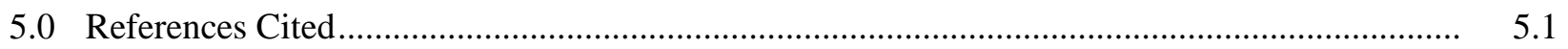

Appendix - Composite Borehole Logs for VOC Investigation and Limited Field Investigation Drilling .................................................................................................. A. 


\section{Figures}

1.1 Trichloroethene Concentrations in the Upper Portion of the Unconfined Aquifer in the Vicinity of the 300 Area During 2007 ..................................................................................... 1.2

1.2 Groundwater Monitoring Locations in the 300 Area ............................................................

1.3 Trichloroethene in Samples Collected During the Limited Field Investigation and Volatile Organic Compound Investigation, 300 Area............................................................. 1.4

1.4 Path Forward Logic Diagram for 300 Area VOC Characterization......................................... 1.4

2.1 Locations for Cross Sections .......................................................................................... 2.2

2.2 Cross Section Southwest to Northeast Across the 300 Area.................................................. 2.3

2.3 Cross Section West to East Across the 300 Area ..................................................................... 2.4

2.4 Cross Section Northwest to Southeast Across the 300 Area ..................................................... 2.5

2.5 Principal Hydrogeologic Units Beneath the 300 Area ......................................................... 2.10

2.6 Gravel-Dominated Sediment of the Hanford Formation.......................................................... 2.13

2.7 Relatively Finer-Grained Sediment Within Ringold Formation Unit E.................................. 2.14

2.8 Sandy Gravel Sediment of Ringold Formation Unit E........................................................... 2.15

2.9 Contact Between Ringold Formation Gravelly Sediment and the Underlying Silty Sediment of the Ringold Formation Lower Mud ................................................................... 2.16

2.10 Elevation of the Contact Surface Between the Hanford and Ringold Formations Beneath

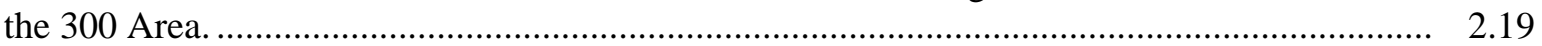

2.11 Cross Section North to South Along the 300 Area Shoreline............................................... 2.21

2.12 Riverbed Adjacent to 300 Area Where Saturated Hanford Formation Is Incised

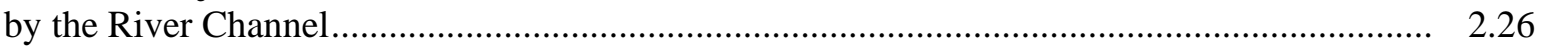

2.13 Perspective View of Contact Between Hanford and Ringold Formations Beneath

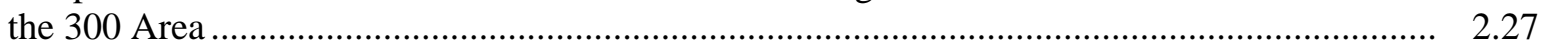

3.1 Tetrachloroethene in Groundwater from Release on November 4, 1982 _................................ 3.2

3.2 Tetrachloroethene in Groundwater from Release on July 6, 1984 ........................................ 3.3

3.3 Increased Tetrachloroethene Concentrations in 300 Area Groundwater During 1998 ............... 3.3

3.4 Inferred Extent of TCE Contamination in the Finer-Grained Interval of Ringold Formation Unit E............................................................................................ 3.5

3.5 Cis-1,2-Dichloroethene Concentrations in Groundwater at Well 399-1-16B .......................... 3.9 


\section{Tables}

1.1 Summary of LFI and VOC Investigation Drilling Information ........................................... 1.7

2.1 Volatile Organic Compounds in Groundwater Samples Collected During Limited Field Investigation Drilling .......................................................................................... 2.6

2.2 Volatile Organic Compounds in Groundwater Samples Collected During VOC

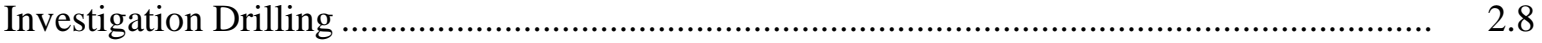

2.3 Summary Information on Major Stratigraphic Units ..................................................... 2.11

2.4 Summary of Hydraulic Test Results for LFI and VOC Investigation Boreholes....................... 2.16

2.5 Volatile Organic Compounds in Samples from Aquifer Tubes ................................................ 2.22

2.6 Results for Columbia River Water Samples Collected Offshore of the South

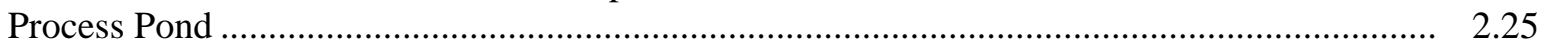





\subsection{Introduction}

In 2006, during an investigation involving uranium in the subsurface at the Hanford Site 300 Area, unexpectedly high concentrations of volatile organic compounds (VOCs) were discovered in groundwater samples collected at two of the four characterization boreholes (Williams et al. 2007). The samples were obtained during drilling and came from a stratigraphic interval in the unconfined aquifer that is not monitored by the existing well network. The occurrences appeared to be restricted to an interval of relatively fine-grained sediment within the Ringold Formation. The concentrations observed exceed the U.S. Environmental Protection Agency (EPA) drinking water standards by as much as two orders of magnitude.

Volatile organic compounds in groundwater beneath the 300 Area are detected frequently but at concentrations lower than the drinking water standards. Figure 1.1 shows the areal distribution of one commonly used organic solvent in shallow groundwater, i.e., near the water table, as observed in samples from monitoring wells during 2007. The plume apparently results from multiple sources, which include local sources associated with past disposal practices as well as contaminated groundwater that migrates into the 300 Area from the southwest. The general characteristics of this plume have not changed appreciably during the past decade or so (Peterson et al. 2005, Table 2.6). Continuing supply from remnant inventories in the subsurface within the 300 Area also is possible, as organic solvents were used extensively during the fuels fabrication process. The concentrations observed during the limited field investigation (LFI) in 2006 (Williams et al. 2007) are clearly anomalous with regard to current conditions near the water table and suggest a different conceptual model for their occurrence and persistence.

In response to this discovery, a strategy to further characterize VOC contamination in the finergrained stratigraphic interval was developed that included additional drilling. The work involved drilling and characterization activities at four new locations in the vicinity of the initial discovery. The drilling was conducted between April and November 2007. Figure 1.2, a monitoring site location map for the 300 Area, shows the locations of characterization boreholes drilled as part of the LFI for uranium as well as the subsequent boreholes drilled as part of the VOC investigation. To illustrate the concentrations of trichloroethene encountered during the LFI and the first of the VOC investigation boreholes, a schematic cross section is shown in Figure 1.3. This report presents the new information obtained since the LFI regarding VOC contamination in the finer-grained interval of Ringold Formation sediment within the unconfined aquifer.

\subsection{Scope of Volatile Organic Compound Investigation}

A sampling and analysis instruction was prepared in March 2007 that presented an investigative strategy for characterizing the nature and extent of VOC contamination in a portion of the unconfined aquifer (Kooiker et al. 2007). The strategy included drilling a second borehole adjacent to one of the earlier LFI boreholes, for several reasons: a) to confirm the presence of contamination in the same stratigraphic interval, and b) to determine whether contamination extends to depths greater than those penetrated by the earlier borehole. Also, historical documents relating to the use, storage, and disposal of degreasing agents and solvents would be reviewed with the intent of uncovering evidence for potential source locations. Finally, a path forward was proposed for activities subsequent to the drilling of the confirmation borehole (Figure 1.4). 


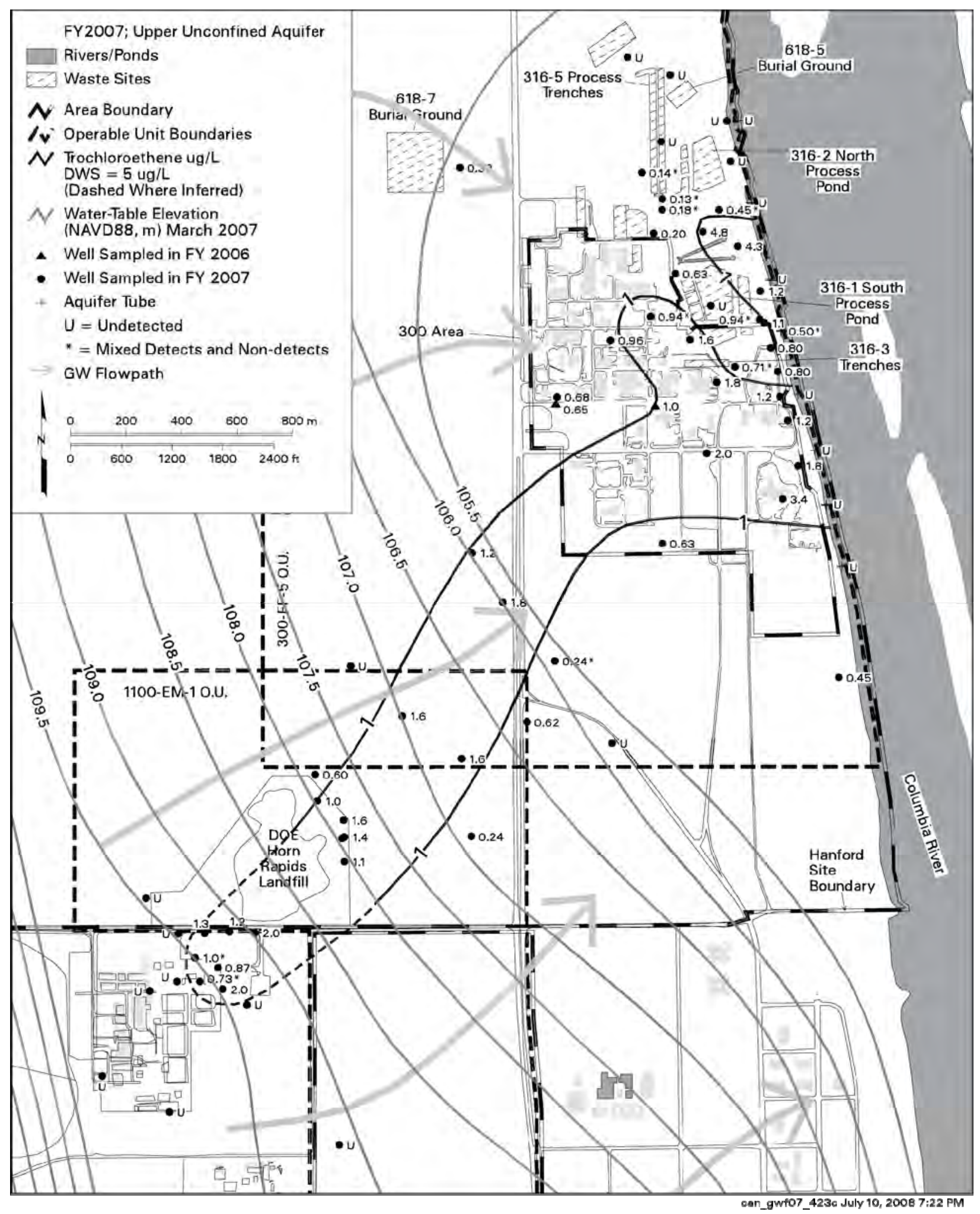

Figure 1.1. Trichloroethene Concentrations in the Upper Portion of the Unconfined Aquifer in the Vicinity of the 300 Area During 2007 (modified from Luttrell and Webber 2008, Figures 2.13-2 and 2.13-3) 


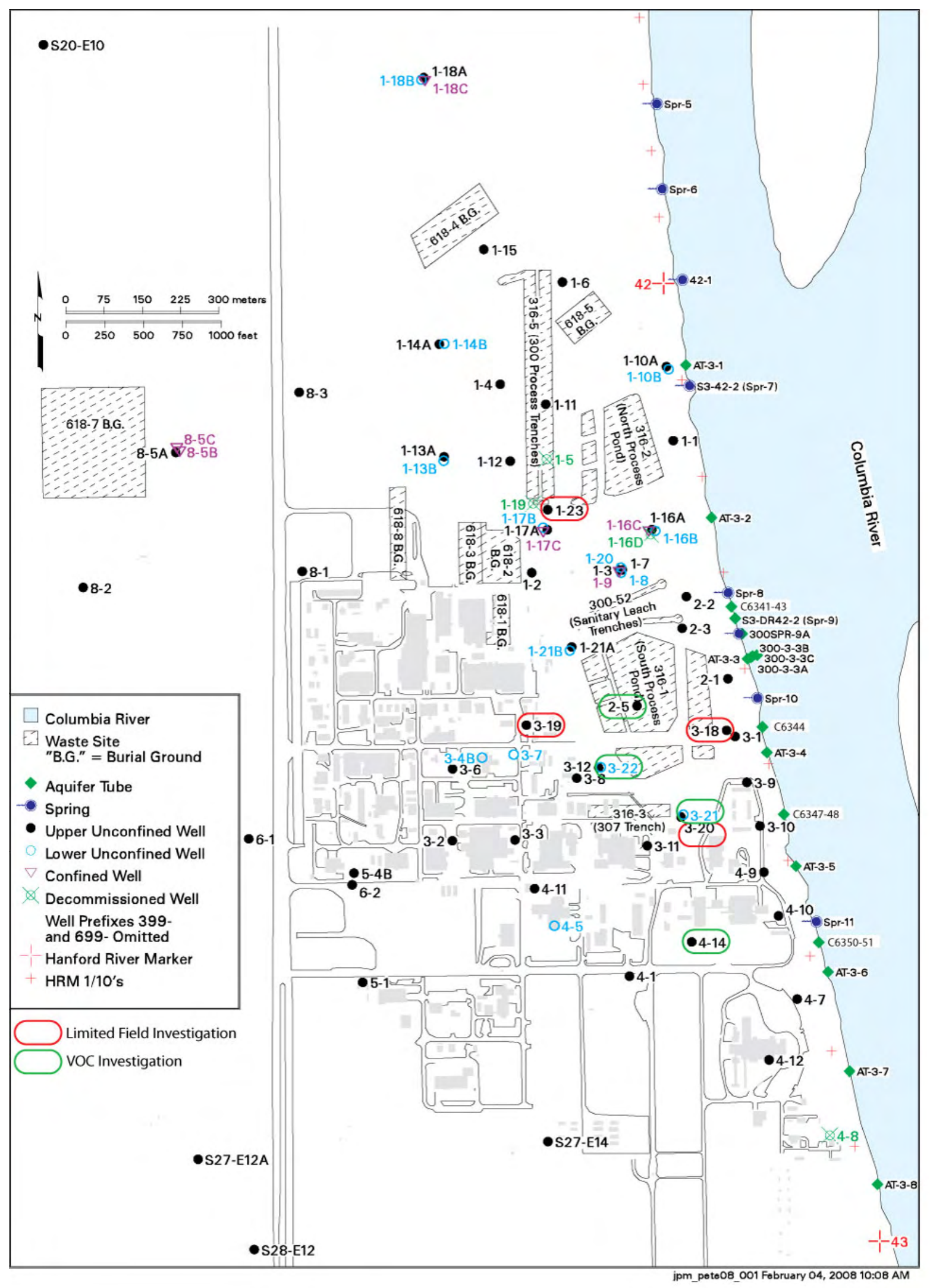

Figure 1.2. Groundwater Monitoring Locations in the 300 Area 


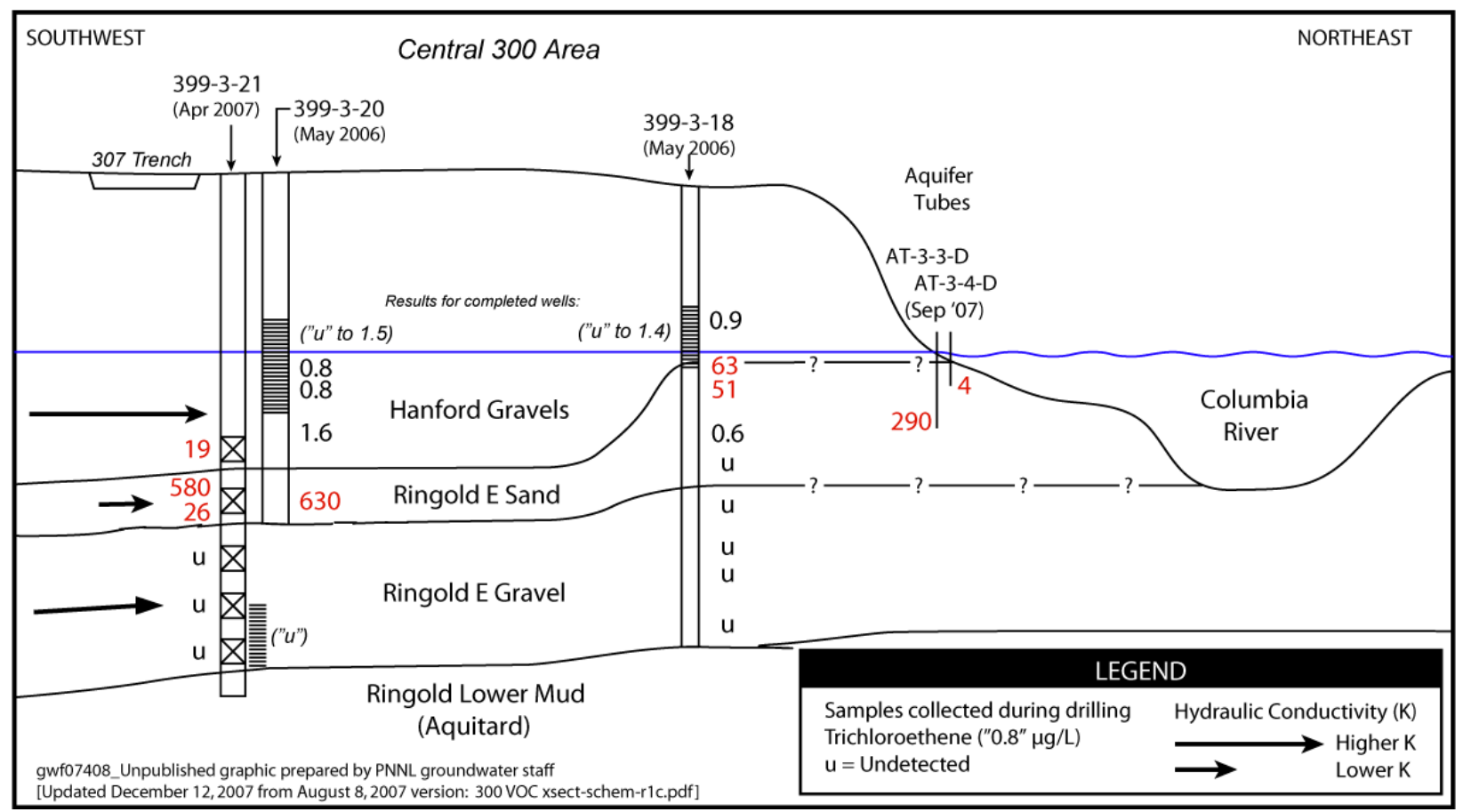

Figure 1.3. Trichloroethene in Samples Collected During the Limited Field Investigation and Volatile Organic Compound Investigation, 300 Area (Peterson and Lindberg 2008)

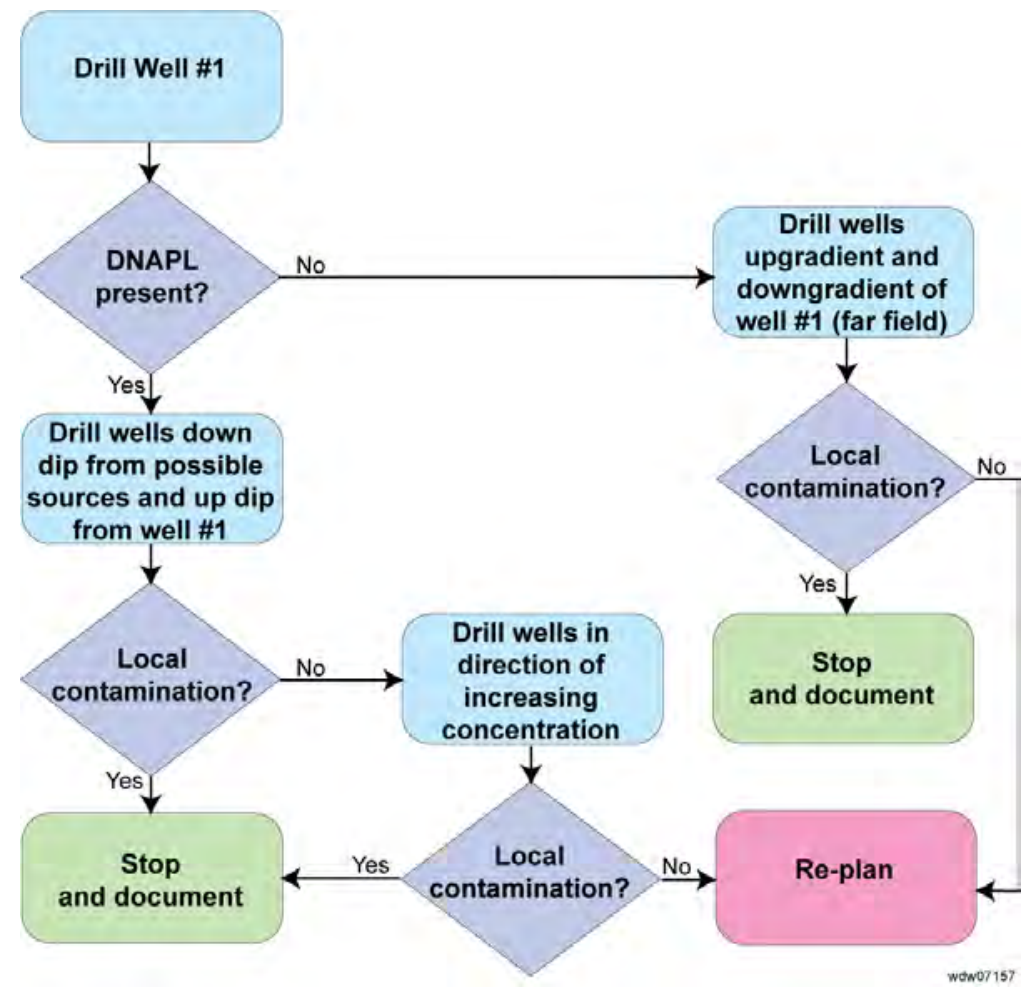

Figure 1.4. Path Forward Logic Diagram for 300 Area VOC Characterization 
Drilling at the confirmation borehole site (399-3-21) confirmed the presence and levels of contamination. No direct evidence for a dense, nonaqueous phase liquid (DNAPL) was uncovered, so the path forward included drilling three additional characterization boreholes in up- and down-gradient directions from the principal discovery location, i.e., in the vicinity of borehole 399-3-20. Contamination appears to be localized in the region immediately to the south and east of the former South Process Pond and east of the 307 Trench. Consequently, a decision has been made to stop the field characterization activities and document what has been learned to date.

\subsection{Environmental Chemistry of Trichloroethene, Tetrachloroethene, and 1,2-Dichloroethene}

Trichloroethene (TCE), tetrachloroethene (PCE), and 1,2-dichloroethene (DCE) are VOCs that are within a larger class of organic compounds known as halogenated hydrocarbons. Halogenated hydrocarbons are common contaminants in the environment because of their widespread use in industry, notably TCE and PCE as degreasing agents (Cohen and Mercer 1993). They are characterized by a relatively high density (specific gravity), low viscosity, and toxicity to humans. TCE and PCE, along with carbon tetrachloride $\left(\mathrm{CCL}_{4}\right)$, are among the more mobile halogenated hydrocarbons in the environment as a nonaqueous phase liquid because of their relatively high density and low viscosity. TCE is moderately soluble in water. It does not accumulate to significant levels in plants, animals, or fish; however it does affect human health by reason of its toxic effects on the major human physiologic systems and to organs, especially the liver and kidneys (ANL 2007) (http://www.ead.anl.gov/pub).

The following general information on these organic compounds has been extracted from the EPA website for its Technology Innovation Program (http://www.clu-in.org/contaminantfocus), unless otherwise cited. Because of volatility, TCE and PCE as liquids do not persist when released to the air, and residence time is measured in days. When released to the ground, some will evaporate; the remainder percolates into the soil. Percolation of TCE and PCE as nonaqueous phase liquids is governed by three factors: 1) density and viscosity, 2) total amount disposed, and 3) subsurface hydraulic properties and conditions. These compounds are not expected to bioaccumulate. Once dissolved, TCE and PCE can adsorb to sediment with moderate level of partitioning as compared to partitioning of other organic compounds. Hydrolysis of TCE, PCE, and other chlorinated ethene compounds is very slow at normal groundwater temperatures.

Under some conditions, TCE and PCE are abiotically degraded to ethene by reduced iron, with no persistent chlorinated intermediate compounds. If the subsurface environment is anaerobic (i.e., low oxygen) and an appropriate electron donor for bacteria is available, reductive dechlorination of TCE and PCE may occur whereby these compounds are sequentially reduced to form DCE isomers, vinyl chloride (VC), and then ethene. Some bacteria can catalyze reductive dechlorination to only DCE; DCE is then persistent under anaerobic conditions. Similarly, some conditions lead to VC as a persistent byproduct. DCE and VC can be degraded in the presence of dissolved oxygen and, in some cases, through coupling with iron reduction processes. When TCE is released to surface water, it volatilizes within hours to several days, depending on the turbulence of the surface water.

TCE and PCE are immiscible in water and are more dense than water, so if large quantities of pure chemical are disposed to the ground or to an injection well, they will sink further as a DNAPL. Some of the chemical will continue to evaporate in the vadose zone and to dissolve in groundwater, although

separate phase contamination may persist in the vadose zone pore space and in the aquifer. To determine 
whether DNAPL is present, analysis of the dissolved concentrations can provide indirect evidence. If the concentration of the contaminant dissolved in groundwater is greater than $1 \%$ of the pure phase solubility, it suggests the presence of a DNAPL (Cohen and Mercer 1993). For TCE, the pure phase solubility is $1,100,000 \mu \mathrm{g} / \mathrm{L}$, so $1 \%$ of that value is $11,000 \mu \mathrm{g} / \mathrm{L}$. For PCE, the pure phase solubility is $150,000 \mu \mathrm{g} / \mathrm{L}$, and $1 \%$ of that value is $1,500 \mu \mathrm{g} / \mathrm{L}$. These threshold values are much higher than those observed during the LFI and VOC investigation (discussed further in Section 3), so a DNAPL source for the contamination observed in groundwater is not suspected.

\subsection{Drilling and Characterization Methodology}

A cable-tool rig was used to drill the four boreholes used as part of the VOC investigation (see Figure 1.2 for locations). The boreholes were located in up- and down-gradient groundwater flow directions from well 399-3-20, the location where the highest concentrations of TCE were observed during the LFI. Drilling information associated with the VOC investigation boreholes drilled is presented in Table 1.1 (information for the earlier LFI boreholes is included for completeness). Characterization activities conducted during drilling included geologic descriptions by the well-site geologist, collection of sediment and groundwater samples, depth-discrete aquifer hydraulic tests, and spectral gamma and neutron moisture geophysical logging. Rapid turn-around analyses for VOCs in groundwater samples collected during drilling were done to help with a) decisions involving characterization activities while the borehole was proceeding, and b) the final design for completing each borehole as a groundwater monitoring well. The rapid-turnaround results, which were conducted in a PNNL laboratory, were consistent with the subsequent results from an offsite analytical laboratory for the same samples.

New borehole 399-3-21 was the first to be drilled and was located adjacent to LFI borehole 399-3-20. The objectives were to confirm the elevated levels of TCE in the finer-grained interval within Ringold Formation Unit E observed earlier, and to further characterize the upper and previously undefined lower portions of the unconfined aquifer. (Note: LFI borehole 399-3-20 did not extend below the finer-grained interval). The data acquired from borehole 399-3-21 confirmed the earlier observation of elevated TCE concentrations in the interval of interest, and also that contamination is constrained to that interval at that location. The borehole was subsequently completed as a monitoring well in the lower portion of the unconfined aquifer (i.e., below the finer-grained interval and near the contact with the top of the Ringold Formation lower mud unit), thus forming a shallow/deep unconfined aquifer monitoring well pair with adjacent well 399-3-20.

Three additional characterization boreholes were subsequently drilled in the vicinity of the 399-3-20/ 399-3-21 well pair. Borehole 399-2-5 was drilled next at a location 230 meters north-northwest of the well pair and within the footprint of the former South Process Pond. Borehole 399-4-14 was drilled $\sim 250$ meters south of the well pair, and finally 399-3-22 was drilled 175 meters to the northwest of the well pair. The relatively finer-grained interval within Ringold Formation Unit $\mathrm{E}$ was encountered at all of these borehole locations. However, the lithologies encountered within the interval varied among the locations, and TCE contamination was detected at only the initial well pair location.

Grab samples collected from the core barrel drive shoe at approximately 1.5-meter (5-foot) depth intervals were used to describe these lithologies as they were encountered in the boreholes. Split-spoon core samples also were collected, although not continuously throughout each borehole. Split-spoon sampling included 10 cores from 399-2-5, 6 cores from 399-4-14, and 7 cores from 399-3-22. The field descriptions are recorded on the geologist's borehole logs, which are provided in a borehole summary 
report (Horner 2008, Appendices A-K) and in the Hanford Well Information System (HWIS). The sample data provide visual confirmation of the relative depths and zonation (i.e., changes in lithology) for Hanford and Ringold Formation stratigraphic units.

The grab sample and split-spoon core data help to form a detailed lithologic description of individual units, and to determine the hydrogeologic contact boundaries and unit thicknesses. The sample quality and formation representativeness of the sediment grab samples are generally good where complete recovery of cuttings occurred. Information from the discreet-depth core samples was used, along with information from the continuous core obtained earlier from the LFI boreholes, to corroborate information developed from the more frequent sediment grab samples.

Table 1.1. Summary of LFI and VOC Investigation Drilling Information

\begin{tabular}{||c|c|c|c|c|c|c|c||}
\hline \hline $\begin{array}{c}\text { Well Name } \\
\text { (Well ID) }\end{array}$ & $\begin{array}{c}\text { Start Date } \\
\text { for } \\
\text { Drilling }\end{array}$ & $\begin{array}{c}\text { Finish } \\
\text { Date for } \\
\text { Drilling }\end{array}$ & $\begin{array}{c}\text { Northing } \\
(\mathrm{m})\end{array}$ & $\begin{array}{c}\text { Easting } \\
(\mathrm{m})\end{array}$ & $\begin{array}{c}\text { Ground } \\
\text { Surface } \\
(\mathrm{m})\end{array}$ & $\begin{array}{c}\text { Total } \\
\text { Drill } \\
\text { Depth } \\
(\mathrm{m})\end{array}$ & $\begin{array}{c}\text { Completed } \\
\text { Well: } \\
\text { Screened } \\
\text { Interval }\end{array}$ \\
\hline \hline \multicolumn{7}{|c|}{ Limited Field Investigation for Uranium (Williams et al. 2007) } \\
\hline $\begin{array}{c}399-3-18 \\
\text { (C4999) }\end{array}$ & $3 / 9 / 06$ & $3 / 23 / 06$ & $116,019.98$ & $594,464.71$ & 117.680 & 77.75 & Water table \\
\hline $\begin{array}{c}399-1-23 \\
\text { (C5000) }\end{array}$ & $3 / 30 / 06$ & $4 / 12 / 06$ & $116,453.04$ & $594,113.52$ & 115.446 & 75.52 & Water table \\
\hline $\begin{array}{c}399-3-19 \\
(5001)\end{array}$ & $4 / 24 / 06$ & $5 / 3 / 06$ & $116,030.22$ & $594,071.94$ & 120.647 & 80.72 & Water table \\
\hline $\begin{array}{c}399-3-20 \\
\text { (C5002) }\end{array}$ & $5 / 11 / 06$ & $5 / 16 / 06$ & $115,849.70$ & $594,375.42$ & 120.448 & 80.52 & Water table \\
\hline \multicolumn{7}{|c|}{ VOC Investigation (Horner 2008) } \\
\hline $\begin{array}{c}399-3-21 \\
\text { (C5575) }\end{array}$ & $4 / 12 / 07$ & $5 / 15 / 07$ & $115,854.28$ & $594,379.75$ & 121.158 & 81.23 & $\begin{array}{c}\text { Lower part, } \\
\text { unconfined }\end{array}$ \\
\hline $\begin{array}{c}399-2-5 \\
\text { (C5708) }\end{array}$ & $9 / 4 / 07$ & $10 / 5 / 07$ & $116,068.80$ & $594,287.74$ & 115.705 & 75.78 & Water table \\
\hline $\begin{array}{c}399-4-14 \\
\text { (C5707) }\end{array}$ & $10 / 8 / 07$ & $10 / 29 / 07$ & $115,604.70$ & $594,396.18$ & 118.792 & 78.86 & Water table \\
\hline $\begin{array}{l}399-3-22 \\
\text { (C5706) }\end{array}$ & $10 / 31 / 07$ & $11 / 29 / 07$ & $115,947.53$ & $594,217.71$ & 119.241 & 79.31 & $\begin{array}{c}\text { Lower part, } \\
\text { unconfined }\end{array}$ \\
\hline $\begin{array}{l}\text { Northing and easting coordinates are Washington State Plane, South Zone (NAD83); } \\
\text { Ground surface elevation and total drill depths are elevations (NAVD88). }\end{array}$ \\
\hline \hline
\end{tabular}





\subsection{Results}

This section presents the results for VOC analysis of groundwater samples collected during the LFI and subsequent VOC investigation. The analytical results are stored in the Hanford Environmental Information System (HEIS). New stratigraphic information that has evolved because of drilling associated with each of these investigations is also presented, as it pertains to the VOC contamination issue.

\subsection{Contaminant Observations During LFI and VOC Characterization Drilling}

To more easily visualize the distribution of VOC contamination in the unconfined aquifer beneath the 300 Area, several hydrogeologic cross sections have been prepared. An index map showing the locations of the cross sections is presented in Figure 2.1. Cross section A-A' (Figure 2.2) extends from the southwest to the northeast through the boreholes where VOC contamination was initially discovered in the interval of finer-grained sediment within Ringold Formation Unit E. Cross section B-B' (Figure 2.3) extends west-to-east across the former South Process Pond and includes the adjacent Columbia River channel. Cross section C-C' (Figure 2.4) extends from the vicinity of the former 300 Area Process Trenches southeast to the South Process Pond. The analytical results for samples collected during drilling are shown on these cross sections.

For each of the four new boreholes drilled as part of the VOC investigation, a composite borehole log has been prepared that summarizes the detailed information gathered during drilling and sampling. These

logs are included in the Appendix to this report, so that they may be pulled out for reference while reading other portions of the report. Detailed information on drilling results is presented in a separate borehole summary report (Horner 2008). The composite borehole logs for the four earlier LFI boreholes are also included in the Appendix for completeness.

The finer-grained sediment interval within Ringold Formation Unit E is represented by yellow highlighting in the cross sections and composite logs, in the same manner as the interval was represented in the earlier LFI report (Williams et al. 2007). The yellow highlight covers several finer-grained lithofacies that occur in the interval between saturated Hanford formation gravels and the underlying Ringold Formation Unit E gravelly sediment. The finer-grained lithologies include muddy silt, fine sand, medium sand, and coarse sand. The finest of these intervals, when present, is at the top of the sequence. While saturated, it does not yield significant groundwater, so no samples were obtained from this layer.

The analytical results for groundwater samples collected during the LFI project, along with initial results from the completed wells, are listed in Table 2.1. Data are presented in vertical order from top to bottom of the borehole, with the screened interval for the completed well shown in blue highlight. Results for TCE and PCE, along with degradation products DCE and VC, are shown in Table 2.1. Also, the maximum contaminant levels for drinking water and the method detection limits are shown in the column headings. Results that exceed the maximum contaminant level are shown in red. The specific conductance for each water sample also is listed, as that parameter is indicative of the different hydrogeologic units. In similar format, the results for samples collected during the VOC investigation are listed in Table 2.2. 


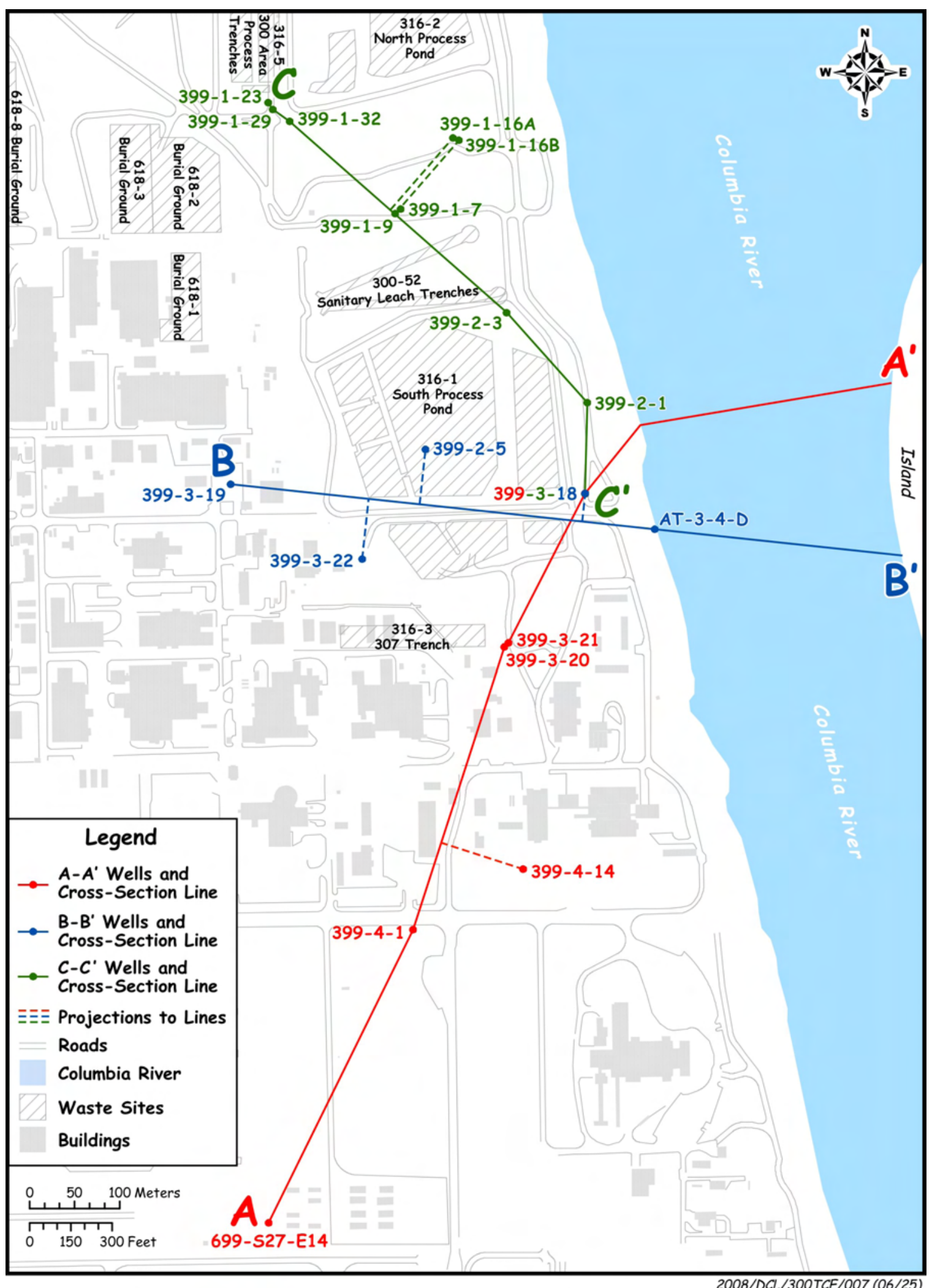

Figure 2.1. Locations for Cross Sections 


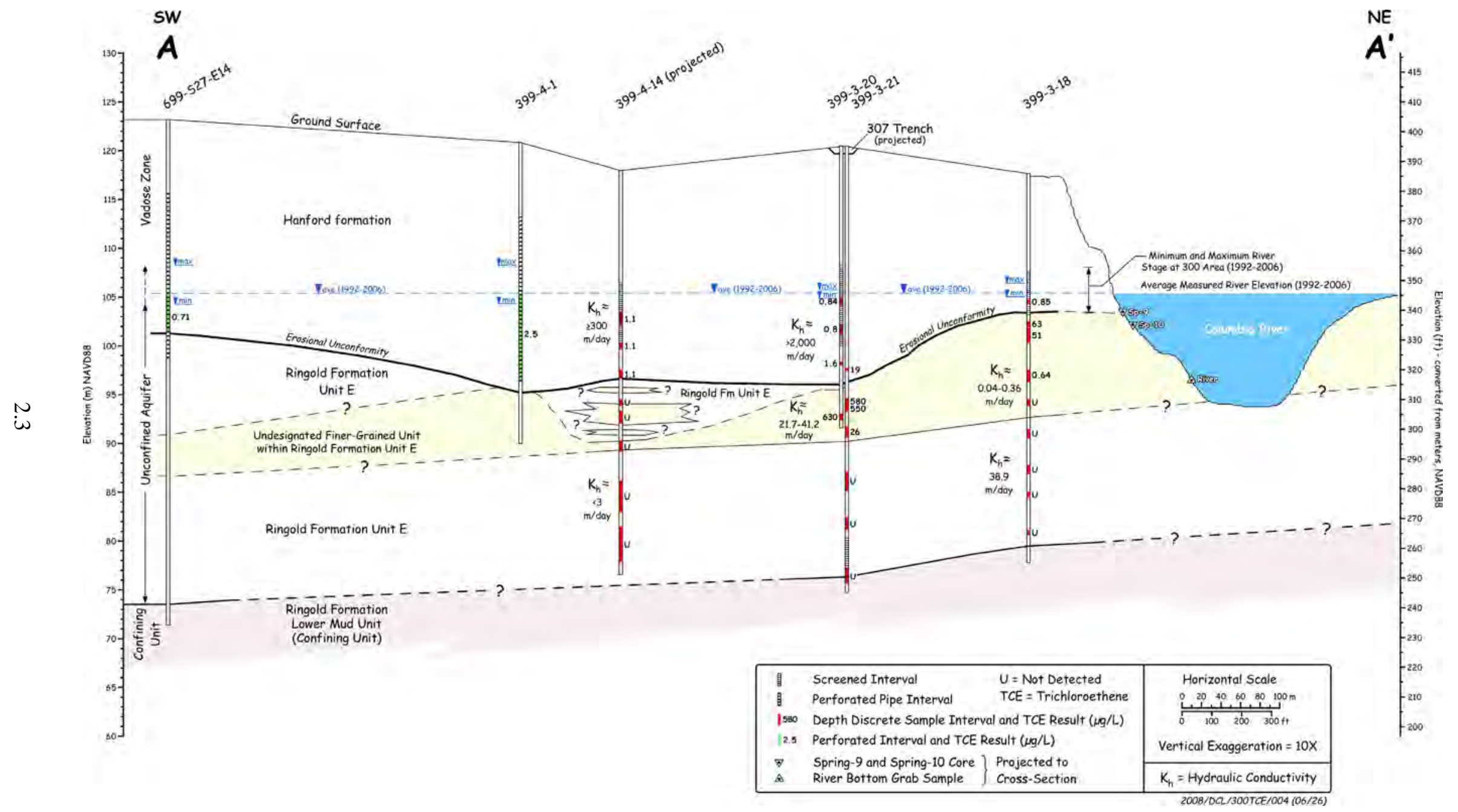

Figure 2.2. Cross Section Southwest to Northeast (A-A') Across the 300 Area 


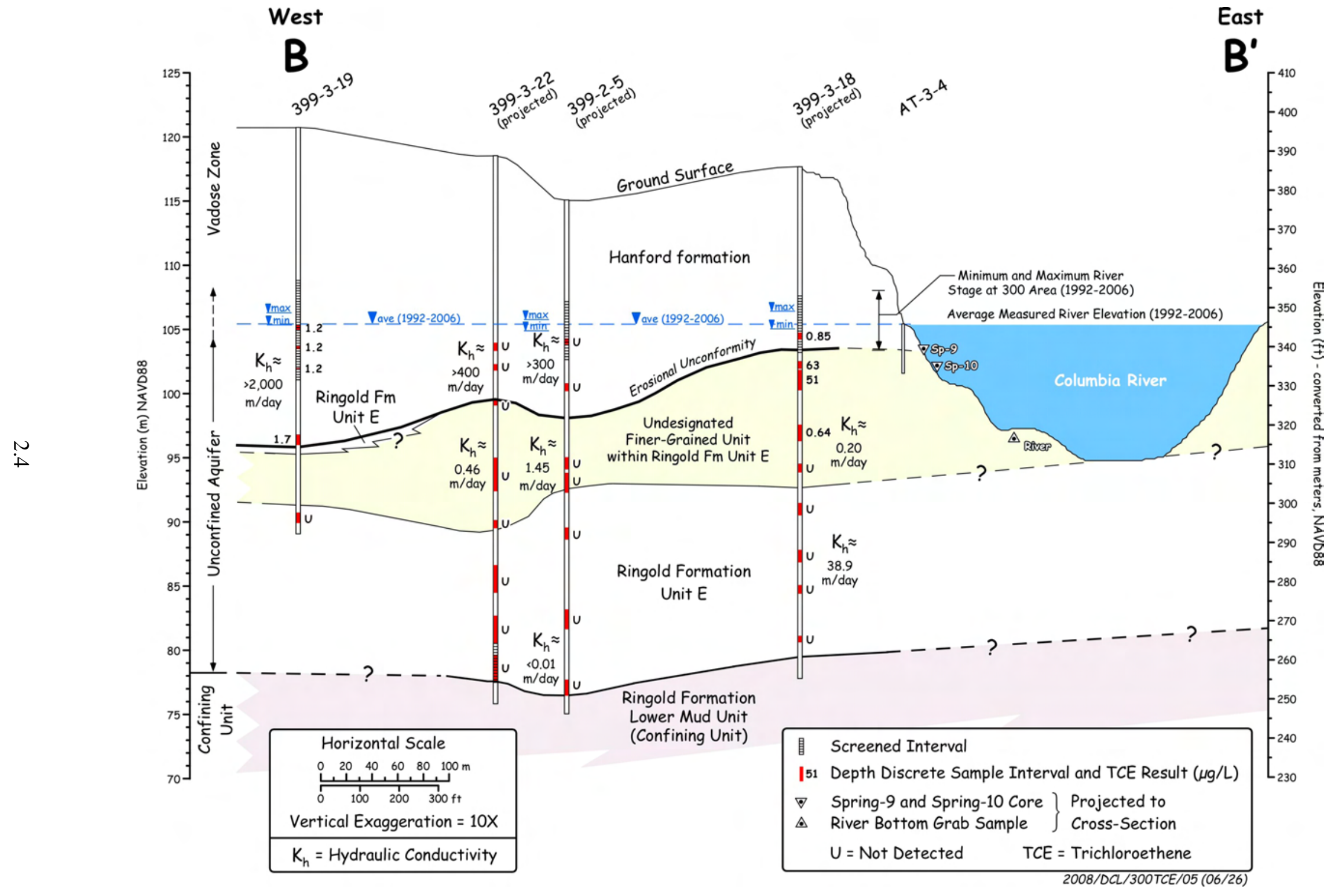

Figure 2.3. Cross Section West to East (B-B') Across the 300 Area 


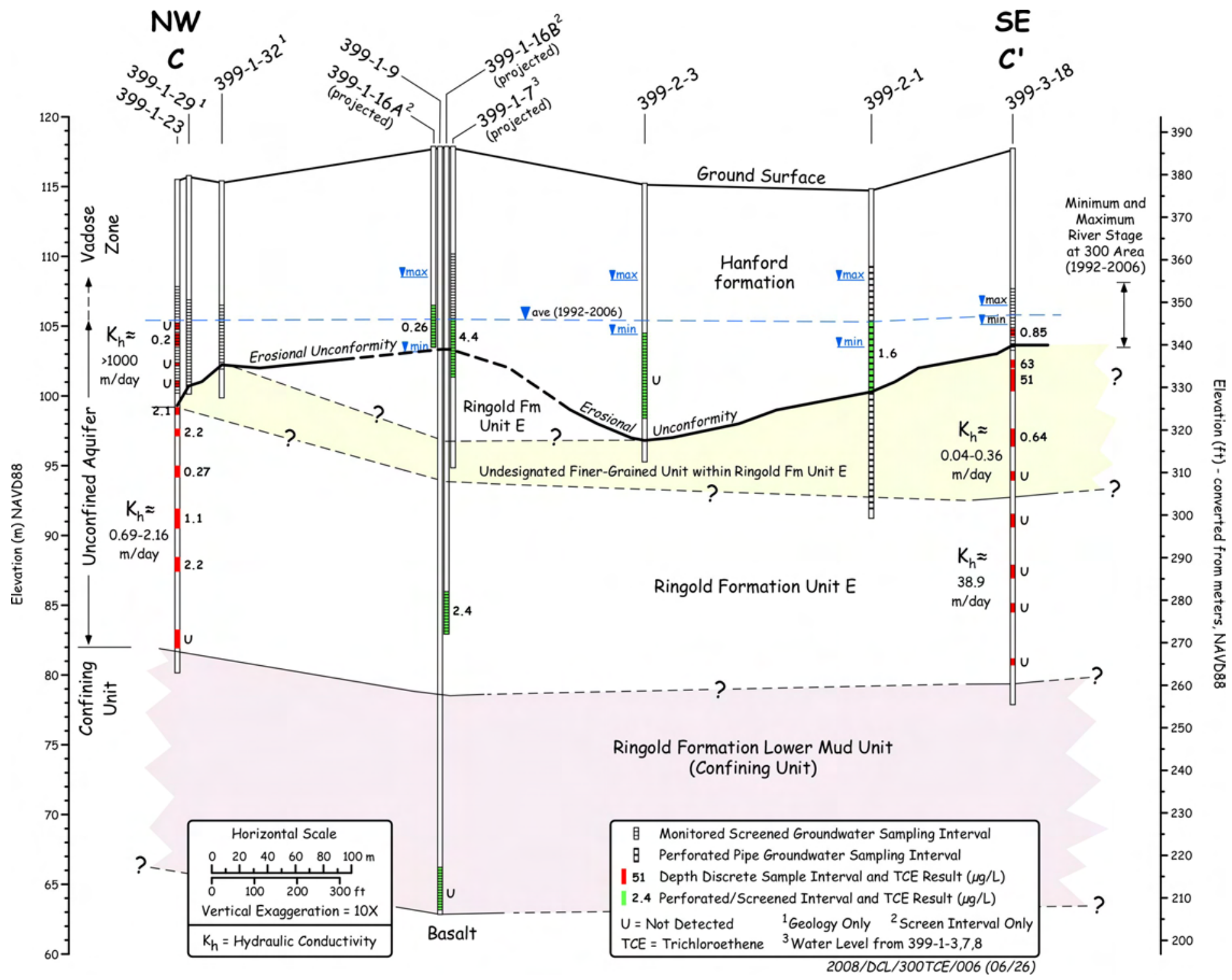

Figure 2.4. Cross Section Northwest to Southeast (C-C') Across the 300 Area 
Table 2.1. Volatile Organic Compounds in Groundwater Samples Collected During Limited Field Investigation Drilling

\begin{tabular}{|c|c|c|c|c|c|c|c|c|c|c|}
\hline $\begin{array}{c}\text { Drilling Sample Location } \\
\text { Designator } \\
\end{array}$ & $\begin{array}{c}\text { Elevation at } \\
\text { Top of Sample } \\
\text { Interval } \\
\text { (m-NAVD88) } \\
\end{array}$ & $\begin{array}{l}\text { Elevation at } \\
\text { Bottom of } \\
\text { Sample } \\
\text { Interval } \\
\text { (m-NAVD88) } \\
\end{array}$ & $\begin{array}{l}\text { Drilling } \\
\text { Sample } \\
\text { Relative to } \\
\text { Final } \\
\text { Screened } \\
\text { Interval } \\
\end{array}$ & $\begin{array}{l}\text { Hydro- } \\
\text { logic } \\
\text { Unit }{ }^{(1)} \\
\end{array}$ & $\begin{array}{c}\text { Sample Collect } \\
\text { Date }\end{array}$ & $\begin{array}{c}\text { Specific } \\
\text { Conduc- } \\
\text { tance } e^{(2)} \\
(\mu \mathrm{S} / \mathrm{cm}) \\
\end{array}$ & 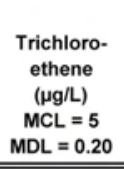 & $\begin{array}{l}\text { Tetrachloro- } \\
\text { ethene } \\
(\mu \mathrm{g} / \mathrm{L}) \\
\mathrm{MCL}=5 \\
\mathrm{MDL}=0.19 \\
\end{array}$ & $\begin{array}{c}\text { Cis-1,2- } \\
\text { Dichloro- } \\
\text { ethene } \\
(\mu \mathrm{g} / \mathrm{L}) \\
\mathrm{MCL}=70 \\
\mathrm{MDL}=0.19 \\
\end{array}$ & $\begin{array}{c}\text { Vinyl Chloride } \\
(\mu g / L) \\
M C L=2 \\
M D L=0.23 \\
\end{array}$ \\
\hline \multicolumn{11}{|c|}{ 399-1-23: Near southern end of former 300 Area Process Trenches (316-5 waste site) } \\
\hline C5000,399-1-23 (1) & 105.2 & 105.1 & Within & $\mathrm{H}_{\text {gravel }}$ & $4 / 3 / 2006$ & nd & U & U & $U$ & U \\
\hline C5000,399-1-23 (2) & 104.5 & 103.6 & Within & $H_{\text {gravel }}$ & 4/4/2006 & 490 & 0.2 & $\mathrm{U}$ & $\mathrm{U}$ & $\mathrm{u}$ \\
\hline C5000,399-1-23 (3) & 102.3 & 102.2 & Within & $\mathrm{H}_{\text {gravel }}$ & $4 / 4 / 2006$ & 492 & $\mathrm{U}$ & $u$ & $U$ & $\mathrm{U}$ \\
\hline C5000,399-1-23 (4) & 101.1 & 100.7 & Within & $\mathrm{H}_{\text {gravel }}$ & $4 / 5 / 2006$ & 485 & U & $U$ & U & U \\
\hline (upper unconfined) & 107.8 & 100.2 & Screen & $\mathrm{H}_{\text {gravel }}$ & $7 / 6 / 2006$ & 485 & 0.2 & $U$ & $U$ & $\mathrm{u}$ \\
\hline (upper unconfined) & 107.8 & 100.2 & Screen & $\mathrm{H}_{\text {gravel }}$ & $9 / 14 / 2006$ & 486 & 0.2 & $U$ & $U$ & U \\
\hline (upper unconfined) & 107.8 & 100.2 & Screen & $\mathrm{H}_{\text {gravel }}$ & $2 / 1 / 2007$ & 475 & 0.2 & $U$ & $U$ & U \\
\hline (upper unconfined) & 107.8 & 100.2 & Screen & $\mathrm{H}_{\text {gravel }}$ & $4 / 19 / 2007$ & 470 & 0.3 & $u$ & $U$ & $u$ \\
\hline (upper unconfined) & 107.8 & 100.2 & Screen & $\mathrm{H}_{\text {gravel }}$ & $9 / 27 / 2007$ & 658 & U & $U$ & $U$ & U \\
\hline (upper unconfined) & 107.8 & 100.2 & Screen & $\mathrm{H}_{\text {gravel }}$ & $1 / 31 / 2008$ & 496 & U & $U$ & $U$ & U \\
\hline (upper unconfined) & 107.8 & 100.2 & Screen & $\mathrm{H}_{\text {gravel }}$ & $3 / 16 / 2008$ & 551 & nyr & nyr & nyr & nyr \\
\hline C5000,399-1-23 (5) & 99.1 & 98.7 & Below & $\mathrm{R}_{\text {gravel }}$ & $4 / 5 / 2006$ & 411 & 2.1 & 0.2 & 3.0 & $U$ \\
\hline C5000,399-1-23 (6) & 97.6 & 97.2 & Below & $\mathrm{R}_{\text {gravel }}$ & $4 / 6 / 2006$ & 396 & 2.2 & U & 15.0 & U \\
\hline C5000,399-1-23 (7) & 95.0 & 94.1 & Below & $R_{\text {gravel }}$ & $4 / 7 / 2006$ & 302 & 0.3 & $u$ & 32.0 & U \\
\hline C5000,399-1-23 (8) & 92.0 & 90.5 & Below & $\mathbf{R}_{\text {gravel }}$ & $4 / 10 / 2006$ & 318 & 1.1 & U & 48.0 & U \\
\hline C5000,399-1-23 (9) & 88.5 & 87.4 & Below & $R_{\text {gravel }}$ & $4 / 11 / 2006$ & 326 & 2.2 & U & 51.0 & U \\
\hline C5000,399-1-23 (10) & 83.3 & 81.9 & Below & $\mathrm{R}_{\text {gravel }}$ & $4 / 17 / 2006$ & 328 & $u$ & $u$ & 57.0 & $\mathrm{U}$ \\
\hline \multicolumn{11}{|c|}{ 399-3-18: Near Columbia River, downgradient of former South Process Ponds (316-1 waste site) } \\
\hline C4999,399-3-18 (1) & 104.7 & 104.7 & Within & $\mathrm{H}_{\text {gravel }}$ & $3 / 14 / 2006$ & 465 & 0.9 & U & U & U \\
\hline C4999,399-3-18 (10) & 104.7 & 103.1 & Within & $\mathrm{H}_{\text {gravel }}$ & $4 / 13 / 2006$ & nd & 0.8 & $U$ & U & U \\
\hline (upper unconfined) & 107.6 & 103.0 & Screen & $\mathrm{H}_{\text {gravel }}$ & $6 / 27 / 2006$ & 200 & 1.4 & U & $U$ & U \\
\hline (upper unconfined) & 107.6 & 103.0 & Screen & $\mathrm{H}_{\text {gravel }}$ & $12 / 8 / 2006$ & 416 & 1.0 & U & U & $u$ \\
\hline (upper unconfined) & 107.6 & 103.0 & Screen & $\mathrm{H}_{\text {gravel }}$ & 4/19/2007 & 250 & 1.1 & $u$ & 0.1 & U \\
\hline (upper unconfined) & 107.6 & 103.0 & Screen & $\mathrm{H}_{\text {gravel }}$ & $7 / 11 / 2007$ & 390 & 1.7 & $U$ & $U$ & U \\
\hline (upper unconfined) & 107.6 & 103.0 & Screen & $\mathrm{H}_{\text {gravel }}$ & $9 / 12 / 2007$ & 473 & $U$ & $U$ & U & U \\
\hline (upper unconfined) & 107.6 & 103.0 & Screen & $\mathrm{H}_{\text {gravel }}$ & $10 / 19 / 2007$ & 478 & na & na & na & na \\
\hline (upper unconfined) & 107.6 & 103.0 & Screen & $\mathrm{H}_{\text {gravel }}$ & $11 / 12 / 2007$ & 468 & na & na & na & na \\
\hline (upper unconfined) & 107.6 & 103.0 & Screen & $\mathrm{H}_{\text {gravel }}$ & $12 / 21 / 2007$ & 456 & $U$ & $U$ & $U$ & U \\
\hline (upper unconfined) & 107.6 & 103.0 & Screen & $\mathrm{H}_{\text {gravel }}$ & $1 / 17 / 2008$ & 407 & na & na & na & na \\
\hline (upper unconfined) & 107.6 & 103.0 & Screen & $\mathrm{H}_{\text {gravel }}$ & $2 / 14 / 2008$ & 452 & na & na & na & na \\
\hline (upper unconfined) & 107.6 & 103.0 & Screen & $\mathrm{H}_{\text {gravel }}$ & $4 / 15 / 2008$ & 473 & nyr & nyr & nyr & nyr \\
\hline C4999,399-3-18 (2) & 103.7 & 102.5 & At bottom & $\mathrm{R}_{\text {sand }}$ & $3 / 14 / 2006$ & 363 & 63.0 & 1.8 & 0.7 & U \\
\hline C4999,399-3-18 (3) & 101.7 & 101.7 & Below & $\mathrm{R}_{\text {sand }}$ & $3 / 15 / 2006$ & 213 & 51.0 & 0.8 & 0.7 & U \\
\hline C4999,399-3-18 (4) & 97.6 & 96.3 & Below & $\mathrm{R}_{\text {sand }}$ & $3 / 16 / 2006$ & 158 & 0.6 & U & U & U \\
\hline C4999,399-3-18 (5) & 94.5 & 93.9 & Below & $\mathrm{R}_{\text {sand }}$ & $3 / 20 / 2006$ & 159 & U & $u$ & U & U \\
\hline C4999,399-3-18 (6) & 91.5 & 90.6 & Below & $R_{\text {gravel }}$ & $3 / 21 / 2006$ & 225 & $U$ & U & U & U \\
\hline C4999,399-3-18 (7) & 87.8 & 86.9 & Below & $R_{\text {gravel }}$ & $3 / 22 / 2006$ & 267 & U & $u$ & 0.9 & U \\
\hline C4999,399-3-18 (8) & 85.1 & 84.5 & Below & $\mathrm{R}_{\text {gravel }}$ & $3 / 22 / 2006$ & 275 & $U$ & U & U & U \\
\hline C4999,399-3-18 (9) & 81.1 & 80.6 & Below & $\mathrm{R}_{\text {gravel }}$ & $3 / 23 / 2006$ & 281 & U & $U$ & 3.0 & $U$ \\
\hline
\end{tabular}


Table 2.1. (contd)

\begin{tabular}{|c|c|c|c|c|c|c|c|c|c|c|}
\hline $\begin{array}{l}\text { Drilling Sample Location } \\
\text { Designator }\end{array}$ & $\begin{array}{c}\text { Elevation at } \\
\text { Top of Sample } \\
\text { Interval } \\
\text { (m-NAVD88) }\end{array}$ & $\begin{array}{l}\text { Elevation at } \\
\text { Bottom of } \\
\text { Sample } \\
\text { Interval } \\
\text { (m-NAVD88) }\end{array}$ & $\begin{array}{l}\text { Drilling } \\
\text { Sample } \\
\text { Relative to } \\
\text { Final } \\
\text { Screened } \\
\text { Interval }\end{array}$ & $\begin{array}{l}\text { Hydro- } \\
\text { logic } \\
\text { Unit }^{(1)}\end{array}$ & $\begin{array}{c}\text { Sample Collect } \\
\text { Date }\end{array}$ & $\begin{array}{l}\text { Specific } \\
\text { Conduc- } \\
\text { tance }{ }^{(2)} \\
(\mu \mathrm{S} / \mathrm{cm})\end{array}$ & $\begin{array}{c}\text { Trichloro- } \\
\text { ethene } \\
(\mu g / L) \\
M C L=5 \\
M D L=0.20\end{array}$ & $\begin{array}{c}\text { Tetrachloro- } \\
\text { ethene } \\
(\mu g / L) \\
M C L=5 \\
M D L=0.19\end{array}$ & $\begin{array}{c}\text { Cis-1,2- } \\
\text { Dichloro- } \\
\text { ethene } \\
(\mu g / L) \\
M C L=70 \\
M D L=0.19\end{array}$ & $\begin{array}{c}\text { Vinyl Chloride } \\
(\mu g / L) \\
M C L=2 \\
M D L=0.23\end{array}$ \\
\hline \multicolumn{11}{|c|}{ 399-3-19: Inland, upgradient from principal liquid waste disposal sites } \\
\hline C5001 399-3-19 (1) & 104.5 & 104.5 & Within & $\mathrm{H}_{\text {gravel }}$ & $4 / 26 / 2006$ & 402 & 1.2 & $U$ & $U$ & $U$ \\
\hline C5001 399-3-19 (2) & 103.1 & 103.0 & Within & $\mathrm{H}_{\text {gravel }}$ & $4 / 27 / 2006$ & 408 & 1.2 & $\mathrm{U}$ & U & $\mathrm{U}$ \\
\hline C5001 399-3-19 (3) & 101.4 & 101.4 & Within & $\mathrm{H}_{\text {gravel }}$ & $4 / 27 / 2006$ & 411 & 1.2 & $\mathrm{U}$ & $U$ & $U$ \\
\hline (upper unconfined) & 108.5 & 100.8 & Screen & $\mathrm{H}_{\text {gravel }}$ & $7 / 6 / 2006$ & 479 & 0.8 & U & U & $U$ \\
\hline (upper unconfined) & 108.5 & 100.8 & Screen & $\mathrm{H}_{\text {gravel }}$ & $9 / 25 / 2006$ & 405 & 1.2 & $U$ & $U$ & $U$ \\
\hline (upper unconfined) & 108.5 & 100.8 & Screen & $\mathrm{H}_{\text {gravel }}$ & $12 / 13 / 2006$ & 422 & 1.8 & U & U & U \\
\hline (upper unconfined) & 108.5 & 100.8 & Screen & $\mathrm{H}_{\text {gravel }}$ & $4 / 19 / 2007$ & 448 & 1.4 & $\mathrm{U}$ & $U$ & $U$ \\
\hline (upper unconfined) & 108.5 & 100.8 & Screen & $\mathrm{H}_{\text {gravel }}$ & $6 / 21 / 2007$ & 464 & 0.6 & $U$ & $U$ & $\mathrm{U}$ \\
\hline (upper unconfined) & 108.5 & 100.8 & Screen & $\mathrm{H}_{\text {gravel }}$ & $9 / 27 / 2007$ & 402 & $\mathrm{U}$ & $U$ & $U$ & $\mathrm{U}$ \\
\hline C5001 399-3-19 (4) & 96.3 & 93.8 & Below & $\mathrm{H}_{\text {gravel }}$ & $4 / 28 / 2006$ & 422 & 1.7 & $\mathrm{U}$ & $U$ & $\mathrm{U}$ \\
\hline C5001 399-3-19 (5) & 90.2 & 89.4 & Below & $\mathrm{R}_{\text {gravel }}$ & $5 / 3 / 2006$ & 318 & 1.4 & $\mathrm{U}$ & $\mathrm{U}$ & $U$ \\
\hline C5001 399-3-19 (6) & no sample & no sample & Below & $\mathrm{R}_{\text {gravel }}$ & no sample & no sample & no sample & no sample & no sample & no sample \\
\hline \multicolumn{11}{|c|}{ 399-3-20: Adjacent to downgradient side of 307 Process Trenches (316-3 waste site) } \\
\hline C5002 399-3-20 (1) & 104.9 & 104.1 & Within & $\mathrm{H}_{\text {gravel }}$ & $5 / 12 / 2006$ & 453 & 0.8 & $U$ & $U$ & $U$ \\
\hline C5002 399-3-20 (2) & 102.2 & 101.2 & Within & $\mathrm{H}_{\text {gravel }}$ & $5 / 12 / 2006$ & 445 & 0.8 & $\mathrm{U}$ & $U$ & $\mathrm{U}$ \\
\hline (upper unconfined) & 108.3 & 100.6 & Screen & $\mathrm{H}_{\text {gravel }}$ & $7 / 6 / 2006$ & 481 & 1.5 & $U$ & $U$ & $U$ \\
\hline (upper unconfined) & 108.3 & 100.6 & Screen & $\mathrm{H}_{\text {gravel }}$ & $9 / 25 / 2006$ & 488 & 1.5 & $\mathrm{U}$ & $U$ & $\mathrm{U}$ \\
\hline (upper unconfined) & 108.3 & 100.6 & Screen & $\mathrm{H}_{\text {gravel }}$ & $12 / 11 / 2006$ & 464 & 1.3 & $U$ & $U$ & $\mathrm{U}$ \\
\hline (upper unconfined) & 108.3 & 100.6 & Screen & $\mathrm{H}_{\text {gravel }}$ & $4 / 19 / 2007$ & 440 & 1.0 & $U$ & $U$ & $\mathrm{U}$ \\
\hline (upper unconfined) & 108.3 & 100.6 & Screen & $\mathrm{H}_{\text {gravel }}$ & $6 / 21 / 2007$ & 471 & 0.6 & $\mathrm{U}$ & $U$ & $\mathrm{U}$ \\
\hline (upper unconfined) & 108.3 & 100.6 & Screen & $\mathrm{H}_{\text {gravel }}$ & $9 / 16 / 2007$ & 491 & $U$ & $U$ & $U$ & $U$ \\
\hline (upper unconfined) & 108.3 & 100.6 & Screen & $\mathrm{H}_{\text {gravel }}$ & $1 / 8 / 2008$ & 456 & $\mathrm{U}$ & $U$ & $\mathrm{U}$ & $U$ \\
\hline C5002 399-3-20 (3) & 98.5 & 98.2 & Below & $\mathrm{H}_{\text {gravel }}$ & $5 / 15 / 2006$ & 463 & 1.6 & $U$ & $U$ & $U$ \\
\hline C5002 399-3-20 (4) & 93.0 & 92.4 & Below & $\mathrm{R}_{\text {sand }}$ & $5 / 16 / 2006$ & 276 & 630.0 & 9.9 & 6.5 & $U$ \\
\hline C5002 399-3-20 (5) & no sample & no sample & Below & unknown & no sample & no sample & no sample & no sample & no sample & no sample \\
\hline C5002 399-3-20 (6) & no sample & no sample & Below & unknown & no sample & no sample & no sample & no sample & no sample & no sample \\
\hline \multicolumn{11}{|c|}{$\begin{array}{l}\text { Color Key: Blue = undetected }(\mathrm{U}) \text {; Black = detected; Red = Exceeds } \mathrm{MCL} \\
\text { Abbreviations: } \mathrm{MCL}=\text { maximum contaminant level for drinking water; } \mathrm{MDL}=\text { method detection limit; } \mathrm{U}=\text { undetected; } \mathrm{nd}=\text { not determined; } \\
\text { na = not analyzed; nyr = not yet reported }\end{array}$} \\
\hline \multicolumn{11}{|c|}{ Ground elevation at Limited Field Investigation borehole sites (m-NAVD88): } \\
\hline \multicolumn{11}{|c|}{$399-1-23$ (115.455); 399-3-18 (117.680); 399-3-19 (120.647); and 399-3-20 (120.448) } \\
\hline
\end{tabular}


Table 2.2. Volatile Organic Compounds in Groundwater Samples Collected During VOC Investigation Drilling

\begin{tabular}{|c|c|c|c|c|c|c|c|c|c|c|}
\hline $\begin{array}{l}\text { Drilling Sample Location } \\
\text { Designator }\end{array}$ & $\begin{array}{c}\text { Elevation at } \\
\text { Top of Sample } \\
\text { Interval } \\
\text { (m-NAVD88) }\end{array}$ & $\begin{array}{c}\text { Elevation at } \\
\text { Bottom of } \\
\text { Sample } \\
\text { Interval } \\
\text { (m-NAVD88) }\end{array}$ & $\begin{array}{l}\text { Drilling } \\
\text { Sample } \\
\text { Relative to } \\
\text { Final } \\
\text { Screened } \\
\text { Interval }\end{array}$ & $\begin{array}{l}\text { Hydro- } \\
\text { logic } \\
\text { Unit }^{(1)}\end{array}$ & $\begin{array}{c}\text { Sample Collect } \\
\text { Date }\end{array}$ & $\begin{array}{l}\text { Specific } \\
\text { Conduc- } \\
\text { tance } e^{(2)} \\
(\mu \mathrm{S} / \mathrm{cm})\end{array}$ & 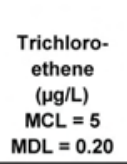 & $\begin{array}{c}\text { Tetrachloro- } \\
\text { ethene } \\
(\mu \mathrm{g} / \mathrm{L}) \\
\mathrm{MCL}=5 \\
\mathrm{MDL}=0.19\end{array}$ & $\begin{array}{c}\text { Cis-1,2- } \\
\text { Dichloro- } \\
\text { ethene } \\
(\mu g / L) \\
M C L=70 \\
M D L=0.19\end{array}$ & $\begin{array}{c}\text { Vinyl Chloride } \\
(\mu g / L) \\
M C L=2 \\
M D L=0.23\end{array}$ \\
\hline \multicolumn{11}{|c|}{ 399-3-21: Adjacent to downgradient side of 307 Process Trenches (316-3 waste site) } \\
\hline C5575-07-(001-008) & 97.6 & 97.5 & Above & $\mathrm{H}_{\text {gravel }}$ & $4 / 17 / 2007$ & 458 & 19.0 & $U$ & $U^{(3)}$ & U \\
\hline C5575-07-(009-024) & 94.5 & 93.2 & Above & $\mathrm{R}_{\text {sand }}$ & $4 / 20 / 2007$ & 303 & 580.0 & 7.0 & $12.0^{(3)}$ & U \\
\hline C5575-07-(025-032) & 91.6 & 90.5 & Above & $\mathrm{R}_{\text {sand }}$ & $4 / 25 / 2007$ & 295 & 26.0 & U & $27.0^{(3)}$ & U \\
\hline C5575-07-(033-040) & 87.0 & 85.0 & Above & $\mathrm{R}_{\text {gravel }}$ & $4 / 30 / 2007$ & 309 & $\mathrm{U}$ & $\mathrm{U}$ & $U^{(3)}$ & $\mathrm{U}$ \\
\hline C5575-07-(041-048) & 82.3 & 81.1 & Above & $\mathrm{R}_{\text {gravel }}$ & $5 / 2 / 2007$ & 318 & $U$ & $U$ & $U^{(3)}$ & $U$ \\
\hline (lower unconfined) & 80.2 & 77.1 & Screen & $\mathrm{R}_{\text {gravel }}$ & $6 / 21 / 2007$ & 318 & $U$ & U & U & $U$ \\
\hline (lower unconfined) & 80.2 & 77.1 & Screen & $\mathrm{R}_{\text {gravel }}$ & $9 / 11 / 2007$ & 360 & U & U & U & $\mathrm{U}$ \\
\hline (lower unconfined) & 80.2 & 77.1 & Screen & $\mathbf{R}_{\text {gravel }}$ & $1 / 8 / 2008$ & 377 & $\mathrm{U}$ & $\mathrm{U}$ & $\mathrm{U}$ & $\mathrm{U}$ \\
\hline (lower unconfined) & 80.2 & 77.1 & Screen & $\mathrm{R}_{\text {gravel }}$ & $4 / 28 / 2008$ & 463 & nyr & nyr & nyr & nyr \\
\hline C5575-07-(049-056) & 77.1 & 76.1 & Below & $\mathbf{R}_{\text {gravel }}$ & $5 / 3 / 2007$ & 382 & U & U & $U^{(1)}$ & U \\
\hline \multicolumn{11}{|c|}{ 399-2-5: South-Central portion of South Process Pond (316-1 waste site) } \\
\hline C5708-07-000 (I-13) & 104.2 & 103.7 & Within & $\mathrm{H}_{\text {gravel }}$ & $9 / 7 / 2007$ & nd & $U$ & U & $U^{(3)}$ & U \\
\hline (upper unconfined) & 107.1 & 102.5 & Screen & $\mathrm{H}_{\text {gravel }}$ & $12 / 28 / 2007$ & 466 & U & $\mathrm{U}$ & $\mathrm{U}$ & $\mathrm{U}$ \\
\hline (upper unconfined) & 107.1 & 102.5 & Screen & $\mathrm{H}_{\text {gravel }}$ & $3 / 16 / 2008$ & 478 & $U$ & $\mathrm{U}$ & $U$ & $\mathrm{U}$ \\
\hline C5708-07-001 (I-16) & 100.7 & 100.1 & Below & $\mathrm{H}_{\text {gravel }}$ & $9 / 13 / 2007$ & nd & $\mathrm{U}$ & $\mathrm{U}$ & $U^{(3)}$ & $\mathrm{U}$ \\
\hline C5708-07-002 (I-17) & 94.9 & 94.0 & Below & $\mathrm{R}_{\text {sand }}$ & $9 / 17 / 2007$ & nd & U & U & $U^{(3)}$ & U \\
\hline C5708-07-003 (I-21) & 93.7 & 92.1 & Below & $\mathrm{R}_{\text {sand }}$ & $9 / 19 / 2007$ & 261 & U & $\mathrm{U}$ & $U^{(3)}$ & $\mathrm{U}$ \\
\hline C5708-07-004 (I-22) & 89.4 & 88.5 & Below & $\mathrm{R}_{\text {gravel }}$ & $9 / 24 / 2007$ & 281 & $\mathrm{U}$ & $\mathrm{U}$ & $U^{(3)}$ & $\mathrm{U}$ \\
\hline C5708-07-000 (I-23) & 83.0 & 81.5 & Below & $\mathrm{R}_{\text {sand }}$ & $9 / 25 / 2007$ & 334 & $\mathrm{U}$ & U & $U^{(3)}$ & U \\
\hline C5708-07-005 (I-24) & 77.5 & 76.3 & Below & $\mathrm{R}_{\text {gravel }}$ & $9 / 27 / 2007$ & 337 & U & U & $U^{(3)}$ & U \\
\hline \multicolumn{11}{|c|}{ 399-4-14: 250 meters south of 307 Process Trenches (center of paleochannel) } \\
\hline (upper unconfined) & 106.4 & 100.3 & Screen & $\mathrm{H}_{\text {gravel }}$ & $1 / 14 / 2008$ & 423 & 1.6 & $U$ & $\mathrm{U}$ & $\mathrm{U}$ \\
\hline (upper unconfined) & 106.4 & 100.3 & Screen & $\mathrm{H}_{\text {gravel }}$ & $3 / 25 / 2008$ & 423 & $U$ & $U$ & $U$ & $U$ \\
\hline C5707-07-000 (I-10) & 103.5 & 102.1 & Within & $\mathrm{H}_{\text {gravel }}$ & $10 / 10 / 2007$ & 446 & 1.1 & $U$ & $U^{(3)}$ & $U$ \\
\hline C5707-07-000 (I-12) & 100.3 & 99.7 & Below & $\mathrm{H}_{\text {gravel }}$ & $10 / 11 / 2007$ & 447 & 1.1 & $\mathrm{U}$ & $U^{(3)}$ & $\mathrm{U}$ \\
\hline C5707-07-000 (I-13) & 97.6 & 96.7 & Below & $\mathrm{H}_{\text {gravel }}$ & $10 / 11 / 2007$ & 441 & 1.1 & $\mathrm{U}$ & $U^{(3)}$ & $\mathrm{U}$ \\
\hline C5707-07-001 (I-14) & 94.5 & 93.9 & Below & $\mathrm{H}_{\text {gravel }}$ & $10 / 12 / 2007$ & 367 & U & $\mathrm{U}$ & $U^{(3)}$ & $\mathrm{U}$ \\
\hline C5707-07-002 (I-15) & 93.3 & 92.1 & Below & $\mathrm{R}_{\text {sand }}$ & $10 / 15 / 2007$ & 324 & $\mathrm{U}$ & $\mathrm{U}$ & $U^{(3)}$ & $\mathrm{U}$ \\
\hline C5707-07-000 (I-17) & 90.3 & 89.2 & Below & $\mathrm{R}_{\text {sand }}$ & $10 / 17 / 2007$ & 311 & U & $\mathrm{U}$ & $U^{(3)}$ & U \\
\hline C5707-07-000 (I-18) & 86.1 & 82.9 & Below & $\mathrm{R}_{\text {gravel }}$ & $10 / 19 / 2007$ & 346 & $U$ & $U$ & $U^{(3)}$ & $U$ \\
\hline C5707-07-000 (I-19) & 81.4 & 77.9 & Below & $\mathbf{R}_{\text {gravel }}$ & $10 / 23 / 2007$ & 374 & U & $U$ & $U^{(3)}$ & U \\
\hline
\end{tabular}


Table 2.2. (contd)

\begin{tabular}{|c|c|c|c|c|c|c|c|c|c|c|}
\hline $\begin{array}{c}\text { Drilling Sample Location } \\
\text { Designator }\end{array}$ & $\begin{array}{c}\text { Elevation at } \\
\text { Top of Sample } \\
\text { Interval } \\
\text { (m-NAVD88) }\end{array}$ & $\begin{array}{c}\text { Elevation at } \\
\text { Bottom of } \\
\text { Sample } \\
\text { Interval } \\
\text { (m-NAVD88) }\end{array}$ & $\begin{array}{l}\text { Drilling } \\
\text { Sample } \\
\text { Relative to } \\
\text { Final } \\
\text { Screened } \\
\text { Interval }\end{array}$ & $\begin{array}{l}\text { Hydro- } \\
\text { logic } \\
\text { Unit }{ }^{(1)}\end{array}$ & $\begin{array}{c}\text { Sample Collect } \\
\text { Date }\end{array}$ & $\begin{array}{c}\text { Specific } \\
\text { Conduc- } \\
\text { tance }{ }^{(2)} \\
(\mu \mathrm{S} / \mathrm{cm})\end{array}$ & $\begin{array}{c}\text { Trichloro- } \\
\text { ethene } \\
(\mu \mathrm{g} / \mathrm{L}) \\
\mathrm{MCL}=5 \\
\mathrm{MDL}=0.20\end{array}$ & $\begin{array}{c}\text { Tetrachloro- } \\
\text { ethene } \\
(\mu \mathrm{g} / \mathrm{L}) \\
\mathrm{MCL}=5 \\
\mathrm{MDL}=0.19 \\
\end{array}$ & $\begin{array}{c}\text { Cis-1,2- } \\
\text { Dichloro- } \\
\text { ethene } \\
(\mu g / L) \\
M C L=70 \\
M D L=0.19\end{array}$ & $\begin{array}{c}\text { Vinyl Chloride } \\
(\mu g / L) \\
M C L=2 \\
M D L=0.23\end{array}$ \\
\hline \multicolumn{11}{|c|}{ 399-3-22: Southwest corner of South Process Pond (316-1 waste site) } \\
\hline C5706-07-00 (I-10) & 103.8 & 103.2 & Above & $\mathrm{H}_{\text {gravel }}$ & $11 / 5 / 2007$ & 415 & $U$ & $U$ & $U^{(3)}$ & $U$ \\
\hline C5706-07-00 (I-11) & 102.2 & 101.7 & Above & $\mathrm{H}_{\text {gravel }}$ & $11 / 5 / 2007$ & 424 & $\mathrm{U}$ & $U$ & $U^{(3)}$ & $U$ \\
\hline C5706-07-00 (I-13) & 99.4 & 99.0 & Above & $\mathrm{H}_{\text {gravel }}$ & $11 / 6 / 2007$ & 412 & $U$ & $U$ & $U^{(3)}$ & $U$ \\
\hline C5706-07-00 (I-15) & 94.8 & 92.3 & Above & $\mathrm{R}_{\text {sand }}$ & $11 / 8 / 2007$ & 279 & $\mathrm{U}$ & $\mathrm{U}$ & $U^{(3)}$ & $\mathrm{U}$ \\
\hline C5706-07-00 (l-16) & 90.0 & 89.4 & Above & $\mathrm{R}_{\text {sand }}$ & $11 / 9 / 2007$ & 300 & $U$ & $U$ & $U^{(3)}$ & $U$ \\
\hline C5706-07-00 (I-18) & 86.5 & 84.3 & Above & $\mathrm{R}_{\text {gravel }}$ & $11 / 13 / 2007$ & 303 & $U$ & $U$ & $U^{(3)}$ & $U$ \\
\hline C5706-07-00 (I-20) & 82.5 & 80.4 & Above & $\mathrm{R}_{\text {gravel }}$ & $11 / 14 / 2007$ & 319 & $U$ & $U$ & $U^{(3)}$ & $U$ \\
\hline C5706-07-00 (I-21) & 79.5 & 77.3 & Within & $\mathrm{R}_{\text {gravel }}$ & $11 / 15 / 2007$ & 348 & U & $U$ & $U^{(3)}$ & $U$ \\
\hline (lower unconfined) & 80.3 & 77.4 & Screen & $R_{\text {gravel }}$ & $12 / 28 / 2007$ & 409 & U & U & U & $U$ \\
\hline (lower unconfined) & 80.3 & 77.4 & Screen & $R_{\text {gravel }}$ & $3 / 25 / 2008$ & 386 & $\mathrm{U}$ & $U$ & $U$ & U \\
\hline \multicolumn{11}{|c|}{$\begin{array}{l}\text { Color Key: Blue = undetected }(U) ; \text { Black = detected; Red = Exceeds } M C L \\
\text { Abbreviations: } M C L=\text { maximum contaminant level for drinking water; } M D L=\text { method detection limit; } U=\text { undetected; nd = not determined; na = } \\
\text { not analyzed; nyr = not yet reported }\end{array}$} \\
\hline \multicolumn{11}{|c|}{$\begin{array}{l}\text { Footnotes: }{ }^{(1)} \mathrm{H}=\text { Hanford gravels, } \mathrm{R}=\text { Ringold Unit E; }{ }^{(2)} \text { Values from HEIS, or field notes if not yet in HEIS; }{ }^{(3)} \text { Result for } \\
\text { 1,2-dichloroethene, total }\end{array}$} \\
\hline \multicolumn{11}{|c|}{ Ground elevation at VOC Investigation borehole sites (m-NAVD88): } \\
\hline \multicolumn{11}{|c|}{$399-3-21$ (120.39); 399-2-5 (115.000); 399-4-14 (117.990); and 399-3-22 (118.471) } \\
\hline
\end{tabular}

\subsection{New Hydrogeologic Information from VOC Investigation Boreholes}

This section provides updates to the hydrogeologic interpretations presented in the earlier LFI report (Williams et al. 2007) for the unconfined aquifer system beneath the 300 Area. The focus is on delineating the thickness and lateral extent (i.e., continuity) of a currently undesignated interval within Ringold Formation Unit E. The update incorporates new data obtained from four additional characterization boreholes that were drilled in the vicinity of the earlier discovery as part of a strategy to determine the extent of that contamination (see Figure 1.2 for locations). The four new characterization boreholes have been completed as monitoring wells, with two having screened openings in the upper portion of the unconfined aquifer, and two in the lower portion.

Geologic characterization activities conducted during the VOC investigation drilling have reinforced some of the previous interpretations developed during the LFI. However, the new results provide additional detail regarding the characteristics of the previously undesignated finer-grained lithologies where VOC contamination was observed at LFI boreholes 399-3-18 and 399-3-20 in 2006. The hydrogeologic interpretations presented in the paragraphs below have resulted from the same process that was developed for the LFI for uranium (Williams et al. 2007). Sediment sample analyses, geologic sample descriptions, depth-discrete groundwater analysis, depth-discrete aquifer hydraulic test analyses, spectral gamma and neutron moisture geophysical logging, and well development data from the four boreholes have collectively contributed to interpreting hydrogeologic conditions at each borehole location. The new information is being used to refine the conceptual model for the hydrogeologic framework beneath the 300 Area. 
The following sections describe the methods used to characterize the hydrogeology, the various lithologies encountered during drilling, preparation of composite borehole logs, hydrologic testing of the aquifer, and an updated conceptual model for the hydrogeologic framework of the area where VOC contamination is likely to be present.

\subsubsection{Borehole Lithology}

The five most dominant lithofacies encountered during drilling, along with their associated hydrologic unit designation, are illustrated in Figure 2.5; their depths and thicknesses are summarized in Table 2.3. Detailed descriptions for each of the five hydrogeologic units, from shallowest (i.e., youngest) to deepest (i.e., oldest), are provided in the following paragraphs. For additional background information on the stratigraphy beneath the 300 Area, refer to Gaylord and Poeter (1991), Swanson et al. (1992), and Lindsey (1995).

Hanford Site - 300 Area

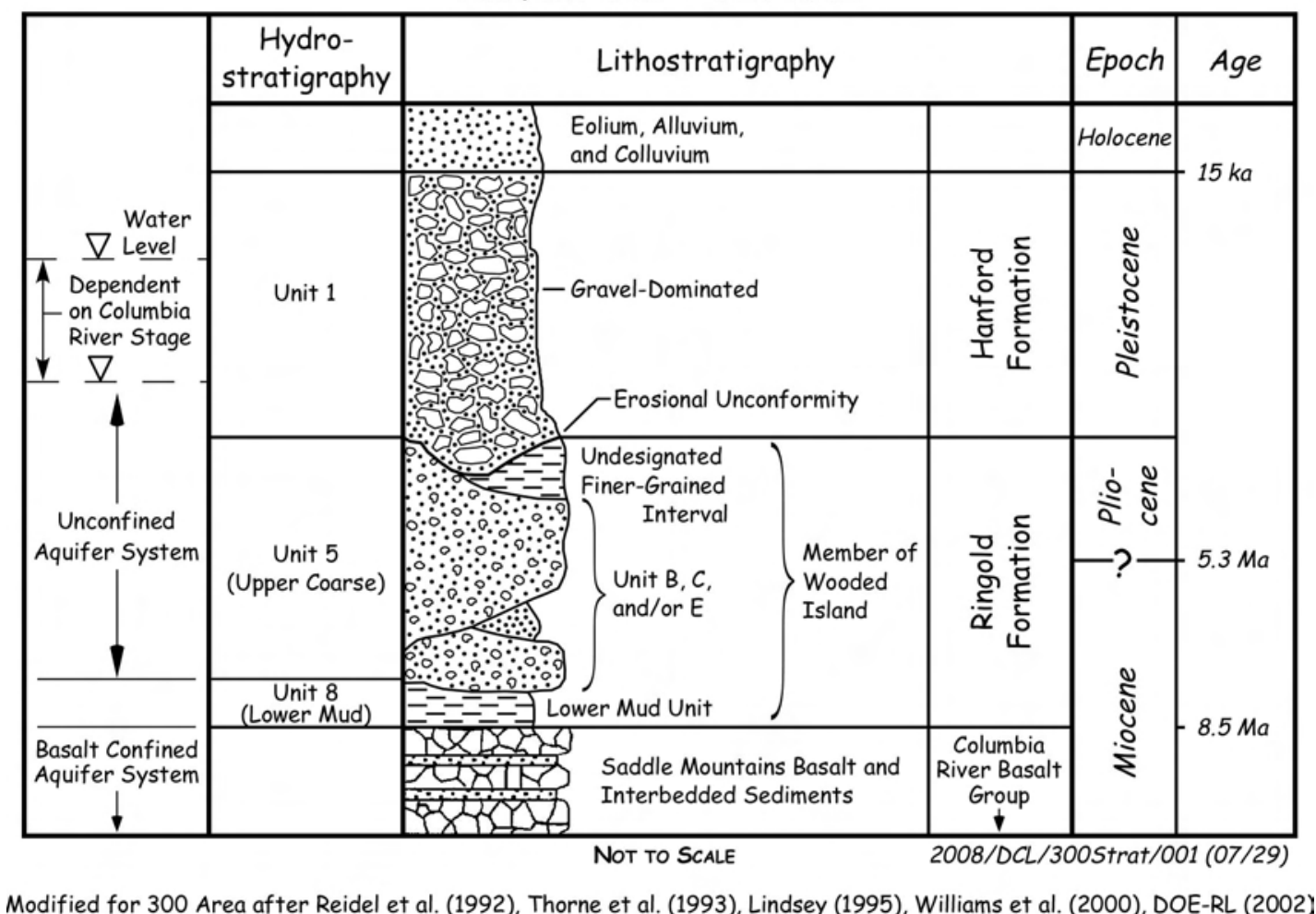

Figure 2.5. Principal Hydrogeologic Units Beneath the 300 Area (Williams et al. 2007)

\section{$\underline{\text { Surficial Sediment }}$}

Recent surficial sediment (Holocene in age) is composed of reworked Hanford formation sandy gravel, eolian silt and sand deposits, and/or anthropogenic backfill of previous excavated sediment, coal plant ash waste, etc. These deposits overlie the area and range in thickness from 0.6 meter ( 2 feet) up to approximately 5.5 meters (18 feet) at the four VOC investigation borehole locations. 


\section{Hanford Formation (Hydrologic Unit 1)}

Gravel-dominated sediment of the Hanford formation comprises the remainder of the vadose zone and the upper, most permeable portion of the unconfined aquifer at all borehole locations. This unit is composed of unconsolidated and clast-supported sediment, with pebble- to boulder-sized gravel, and containing a poorly sorted matrix of fine- to coarse-grained sand. Silt content varies and locally fills most or all matrices between gravel clasts. Occasionally, matrix is missing, which produces an openframework fabric (Figure 2.6). The Hanford formation ranges in thickness from 13.1 meters (43 feet) to $\sim 18.7$ meters (62 feet) at the four borehole locations.

Table 2.3. Summary Information on Major Stratigraphic Units (modified after Horner 2008)

\begin{tabular}{|c|c|c|c|c|c|c|c|c|}
\hline \multirow{2}{*}{$\begin{array}{c}\text { Well Name } \\
\text { (Well ID) } \rightarrow \\
\text { Lithology and } \\
\text { Hydrologic } \\
\text { Unit }^{*}\end{array}$} & \multicolumn{2}{|c|}{$\begin{array}{c}399-3-21 \\
(\mathrm{C} 5575)\end{array}$} & \multicolumn{2}{|c|}{$\begin{array}{c}399-3-22 \\
(\mathrm{C} 5706)\end{array}$} & \multicolumn{2}{|c|}{$\begin{array}{c}399-4-14 \\
\text { (C5707) }\end{array}$} & \multicolumn{2}{|c|}{$\begin{array}{l}399-2-5 \\
\text { (C5708) }\end{array}$} \\
\hline & $\begin{array}{c}\text { Depth } \\
\text { Interval } \\
\text { (feet bgs) }\end{array}$ & $\begin{array}{c}\text { Unit } \\
\text { Thickness } \\
\text { (feet) }\end{array}$ & $\begin{array}{c}\text { Depth } \\
\text { Interval } \\
\text { (feet bgs) }\end{array}$ & $\begin{array}{c}\text { Unit } \\
\text { Thickness } \\
\text { (feet) }\end{array}$ & $\begin{array}{c}\text { Depth } \\
\text { Interval } \\
\text { (feet bgs) }\end{array}$ & $\begin{array}{c}\text { Unit } \\
\text { Thickness } \\
\text { (feet) }\end{array}$ & $\begin{array}{c}\text { Depth } \\
\text { Interval } \\
\text { (feet bgs) }\end{array}$ & $\begin{array}{c}\text { Unit } \\
\text { Thickness } \\
\text { (feet) }\end{array}$ \\
\hline $\begin{array}{c}\text { Surficial } \\
\text { Sediment }\end{array}$ & $0-18$ & 18 & $0-2$ & 2 & $0-7.5$ & 7.5 & $0-13$ & 13 \\
\hline $\begin{array}{c}\text { Hanford fm } \\
\text { coarse gravel } \\
\text { (Unit 1) }\end{array}$ & $18-78$ & 60 & $2-63.5$ & 61.5 & $7.5-67$ & 59.5 & $13-56$ & 43 \\
\hline $\begin{array}{l}\text { Ringold Fm } \\
\text { undesignated } \\
\text { finer-grained } \\
\text { lithologies } \\
\text { (Unit 5) }\end{array}$ & $78-99.5$ & 12.5 & $63.5-96$ & 32.5 & $67-94$ & 27 & $56-73$ & 17 \\
\hline $\begin{array}{l}\text { Ringold Fm } \\
\text { coarse gravel } \\
\text { (Unit 5) }\end{array}$ & $99.5-145$ & 45.5 & $96-135$ & 39 & $94-130$ & 35.5 & $73-125$ & 52 \\
\hline $\begin{array}{c}\text { Ringold Fm, } \\
\text { mud/silt } \\
\text { (Unit 8) }\end{array}$ & $\begin{array}{l}145-150+ \\
(150=\text { TD })\end{array}$ & Unknown & $\begin{array}{l}135-141+ \\
(141=\mathrm{TD})\end{array} \mid$ & Unknown & $\begin{array}{l}130-136+ \\
(136=\mathrm{TD})\end{array} \mid$ & Unknown & $\begin{array}{l}125-131+ \\
(131=\mathrm{TD})\end{array}$ & Unknown \\
\hline $\begin{array}{l}\text { dentified in pa } \\
\text { Abbreviations: }\end{array}$ & $\begin{array}{l}\text { heses. } \\
=\text { below }\end{array}$ & ydrologic & ce; TD = & I drilled d & or use in & undwate & c und & $\begin{array}{l}\text { f four } \\
\text { are also }\end{array}$ \\
\hline
\end{tabular}

There are no easily distinguishable or readily mapped facies/hydrogeologic changes within the vadose zone in the area covered by these boreholes. There are isolated occurrences of older, reworked Ringold Formation sediment, which is distinguished by its more cohesive sediment structure, color, and/or degree of sorting. The reworked Ringold sediment also may contain zones with higher clay and silt content (Bjornstad 2004). Large Ringold rip-up clasts, up to 0.7 meter (several feet) in diameter and composed of pure silt and clay, are occasionally present. One of those clasts, which is at least 0.15 meter ( 0.5 feet) in thickness, was encountered during drilling at 399-2-5, from a depth of 14.3 meters (47 feet) below ground surface within the Hanford formation gravel. 


\subsubsection{Ringold Formation Undesignated Finer-Grained Interval (Hydrologic Unit 5)}

An erosional unconformity separates this interval of sand and silt lithofacies within the Ringold Formation from the overlying Hanford formation in some portions of the 300 Area. These lithofacies include predominantly silt or fine-, medium-, and coarse-grained sand (Figure 2.7). Within this interval, grain size appears to increase with depth. The presence of this relatively finer-grained interval within the generally coarse-grained sediment of the Ringold Formation was confirmed by grab and core samples collected from all four of the VOC investigation boreholes. The finer-grained interval was encountered at or near the Hanford/Ringold contact and ranges in thickness from 4 meters (13 feet) at borehole 399-3-21 to 10 meters (33 feet) at borehole 399-3-22.

\subsubsection{Ringold Formation Coarse Gravel (Hydrologic Unit 5)}

Underlying the relatively finer-grained interval is a coarse gravel lithofacies. It is composed of variably indurated, fluvial gravel, to silty- sandy gravel, and extends to the base of the unconfined aquifer (Figure 2.8). The range in thickness is $\sim 11$ meters (36 feet) to $\sim 16$ meters (52 feet). At some 300 Area locations, this gravel lithofacies may be present also above the relatively finer-grained interval (see cross sections in Figures 2.2 through 2.4).

Differences between the gravelly sediment in the much younger Hanford formation and the coarsegrained portions of the Ringold Formation include a distinct change in basalt content, color, consolidation, and better grain-sorting and grain-roundness in the older Ringold sediments. Other contrasts include significant differences in hydraulic properties as revealed by aquifer tests (e.g., much lower hydraulic conductivity in Ringold than in Hanford sediment), and differences in the total gamma activity of the sediment (e.g., variability in amount of natural potassium-40).

\subsubsection{Ringold Formation Lower Mud (Hydrologic Unit 8)}

The Ringold Formation lower mud unit underlies the Ringold Formation gravelly sediment and is an aquitard that forms the lower boundary of the unconfined aquifer system. This aquitard separates the confined aquifers in the underlying Columbia River Basalt Group from the overlying unconfined aquifer. The lower mud unit contains silty clay to silty sand sediment, and forms a sharp, well-defined contact boundary with the overlying fluvial gravel sediment (Figure 2.9). The VOC investigation boreholes were drilled approximately 1.5 meters (5 feet) into the lower mud at each borehole location, primarily to confirm the presence of the unit and to enable geophysical logging across the contact boundary.

\subsubsection{Depth-Discrete Aquifer Hydraulic Testing Results}

Multiple, depth-discrete aquifer hydraulic tests (slug tests) provide information on unit-specific groundwater flow conditions in various hydrogeologic units throughout the vertical profile of the borehole. These results reveal the general permeability characteristics for the principal hydrogeologic units within the unconfined aquifer system. The aquifer testing conducted as part of the VOC investigation is described in detail in a separate report (Newcomer 2008). That report contains descriptions of the aquifer hydraulic testing performed at each borehole site, a listing of the intervals analyzed in each borehole, and the associated analytical data and their interpretation. A brief summary of the depth discrete best-estimate hydraulic conductivity $\left(\mathrm{K}_{\mathrm{h}}\right)$ values for the respective characterization wells also is provided as a table that is included on the composite borehole log for each site (Figures A.1 through A.4). 


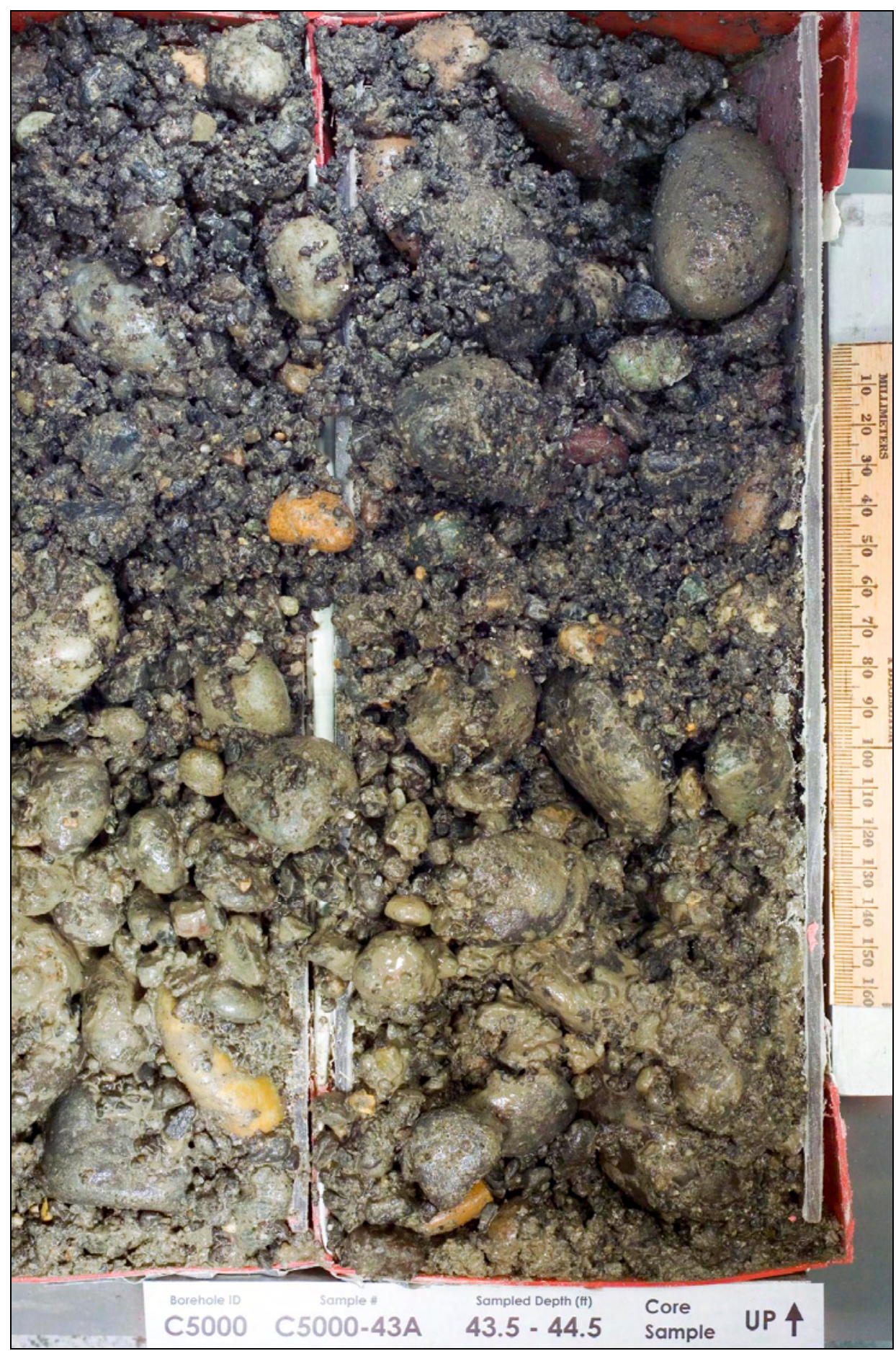

Figure 2.6. Gravel-Dominated Sediment of the Hanford Formation (Hydrologic Unit 1) 


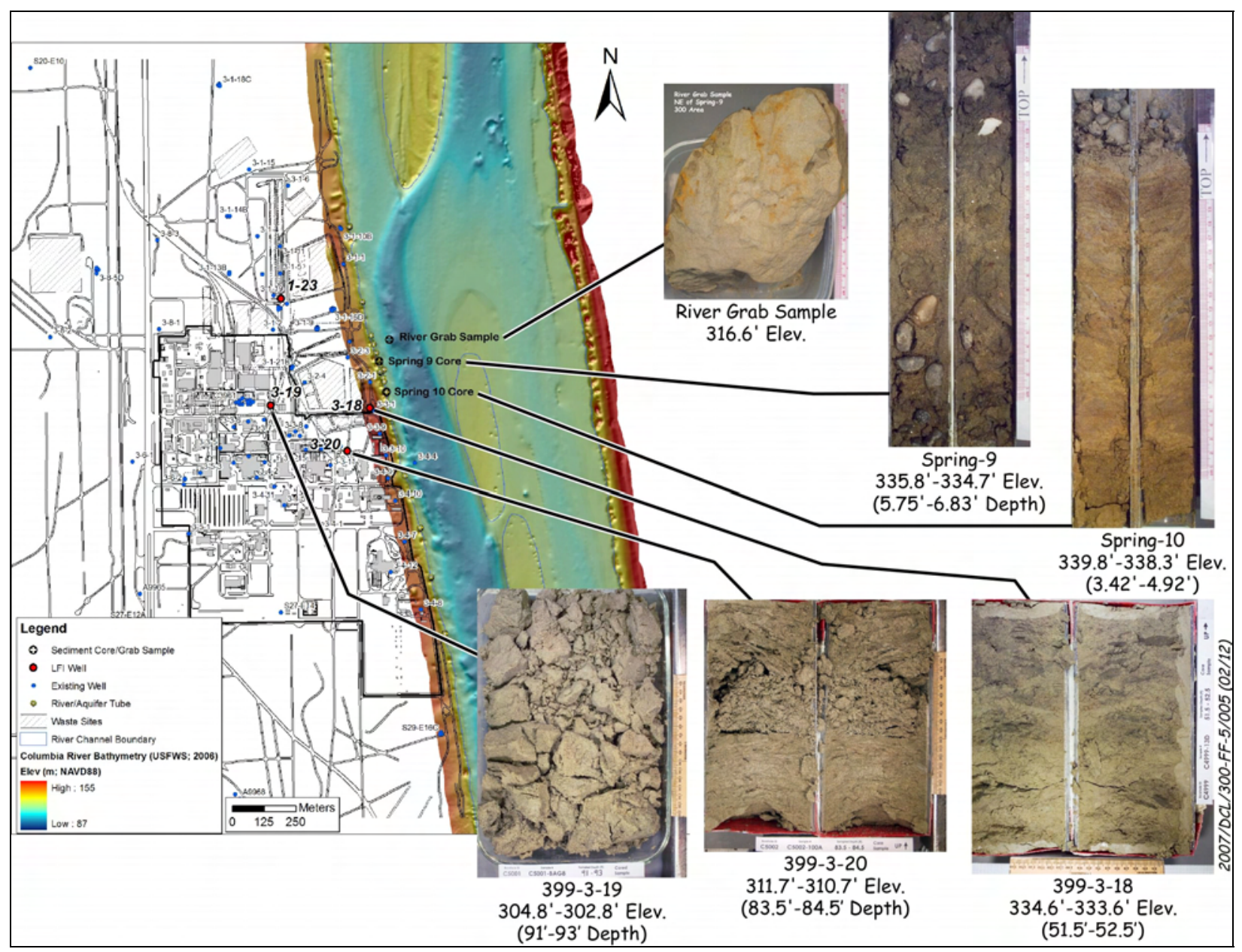

Figure 2.7. Relatively Finer-Grained Sediment Within Ringold Formation Unit E (Hydrologic Unit 5; Figure 3.18 from Williams et al. 2007, p. 3.30)

In general, the vertical distribution and values for hydraulic conductivity in the four VOC investigation boreholes is consistent with the previous LFI borehole test results (Williams et al. 2007). A summary of aquifer test results for the LFI and VOC investigation boreholes is presented in Table 2.4, where a generalized sequence of lithofacies shows how the tests are grouped into the three primary groundwater flow intervals within the unconfined aquifer. All results indicate very high permeability characteristics for the Hanford formation gravel-dominated facies compared to very low-to-moderate permeability for the various Ringold Formation lithofacies. The lowest permeability is revealed by the average hydraulic conductivity $\left(\mathrm{K}_{\mathrm{h}}\right)$ measured in the relatively finer-grained interval of the Ringold Formation, where values are less than 2 meters per day. These low values are in contrast to values in the overlying Hanford formation gravelly sediment, which may exceed 300 meters per day. 


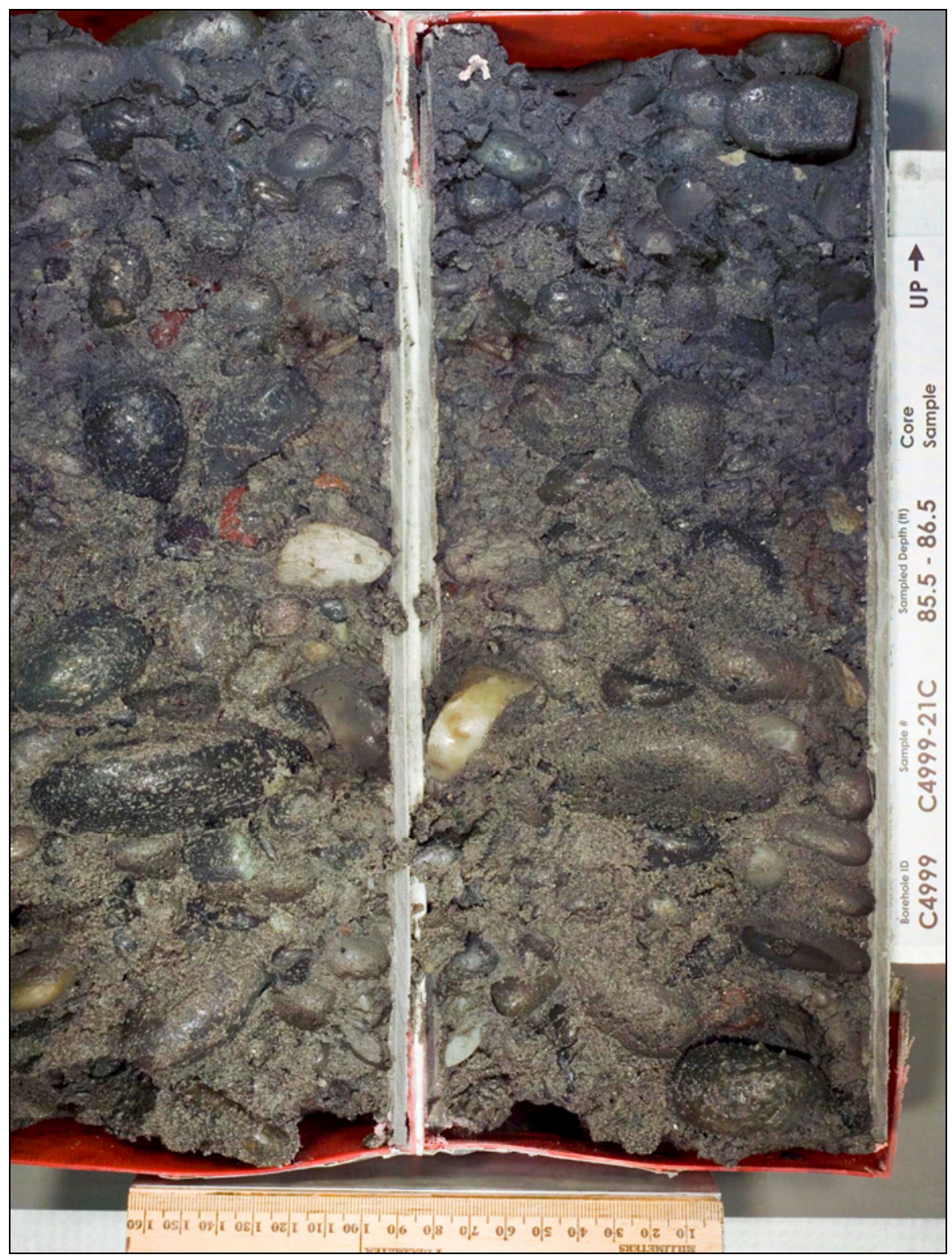

Figure 2.8. Sandy Gravel Sediment of Ringold Formation Unit E (Hydrologic Unit 5)

Groundwater moves laterally much more rapidly within the saturated Hanford formation than in the underlying Ringold Formation. This is particularly true for the relatively finer-grained interval in Ringold sediment where elevated concentrations of TCE have been found. The implication of high permeability in the uppermost portion of the unconfined aquifer (including the water table) is that contaminants currently entering groundwater from the overlying vadose zone are likely to be removed from the aquifer via rapid lateral transit through the saturated Hanford gravels pathway, before there is opportunity to contaminate deeper intervals in the unconfined aquifer. 
Table 2.4. Summary of Hydraulic Test Results for LFI and VOC Investigation Boreholes

\begin{tabular}{|c|c|c|c|c|c|c|c|c|}
\hline \multirow[b]{2}{*}{$\begin{array}{c}\text { Stratigraphic } \\
\text { Formation }\end{array}$} & \multirow{2}{*}{$\begin{array}{c}\text { Vertical Sequence of } \\
\text { Lithofacies } \\
\text { Encountered } \\
\text { (typical) }\end{array}$} & \multicolumn{3}{|c|}{ Limited Field Investigation } & \multicolumn{4}{|c|}{ VOC Investigation } \\
\hline & & $399-3-18$ & $399-3-19$ & $399-3-20$ & $399-3-21$ & $399-2-5$ & $399-4-14$ & $399-3-22$ \\
\hline Hanford & \begin{tabular}{|l|} 
Sandy gravel \\
\end{tabular} & & $\geq 2,000$ & $>2,000$ & 568 & $\geq 300$ & $\geq 300$ & $\geq 400$ \\
\hline Hanford & Sandy gravel & & 2,200 & & no result & & & \\
\hline Ringold & Muddy sandy gravel & & & & & & & \\
\hline Ringold & Mud & & & & & & & \\
\hline Ringold & Muddy sand & & & & & & & \\
\hline Ringold & Sandy mud & & & & & & & \\
\hline Ringold & Fine sand & 0.04 & & & & & & \\
\hline Ringold & Fine-medium sand & 0.36 & & 21.7 & 1.04 & & & 0.61 \\
\hline Ringold & Medium-coarse sand & no result & & 41.2 & no result & 1.73 & no result & \\
\hline Ringold & Coarse sand & & & & & & & \\
\hline Ringold & Silty sandy gravel & & & & 0.27 & & 2.85 & \\
\hline Ringold & Silty sandy gravel & & & & 0.34 & & & \\
\hline Ringold & Silty sandy gravel & 38.9 & & & 2.03 & & 1.12 & 1.51 \\
\hline Ringold & Silty sandy gravel & 3.82 & & & 1.47 & $\leq 0.01$ & & no result \\
\hline Ringold & Clayey silt & & & & Aquitard & & & \\
\hline
\end{tabular}

Notes: Hydraulic conductivity values $\left(\mathrm{K}_{\mathrm{h}}\right)$ in meters per day, as measured using slug tests in individual boreholes. Table assembled from data presented in Williams et al. 2007 (PNNL-16435), Horner 2008

(SGW-36424), and Newcomer 2008 (PNNL-17439). Yellow highlight indicates finer-grained interval of concern.

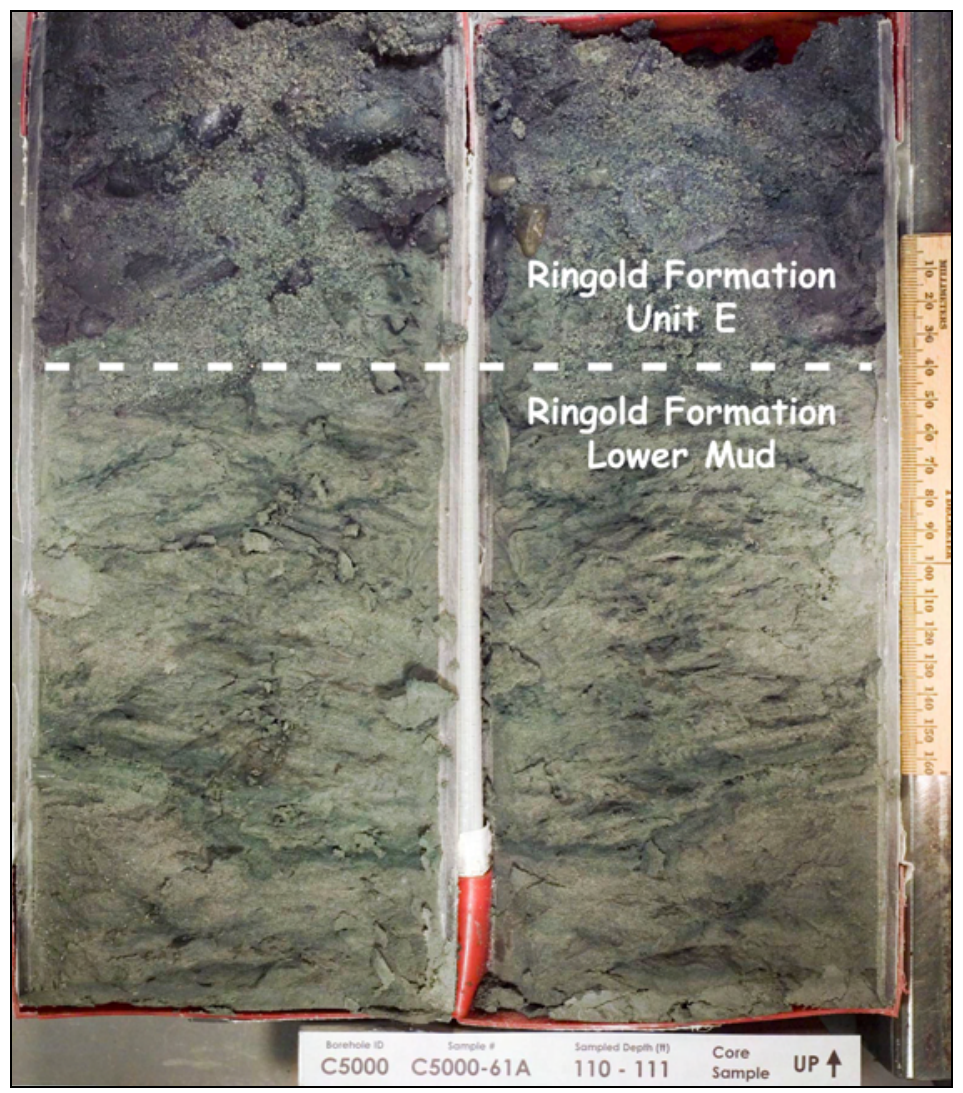

Figure 2.9. Contact Between Ringold Formation Gravelly Sediment and the Underlying Silty Sediment of the Ringold Formation Lower Mud (Hydrologic Unit 8) 


\subsubsection{Spectral Gamma and Neutron Moisture Geophysical Logging}

Spectral gamma logging data are used qualitatively to assist in defining lithologic and hydrogeologic contacts, as well as to screen for man-made radionuclides, such as uranium isotopes associated with fuels fabrication activities during historical operations. The inflections recorded on the geophysical logs were used to corroborate and/or to define more precisely the changes in lithology as described by the well site geologist, for example, sand vs. silt and clay or gravel intervals. The logs also help to precisely locate the water table, and to reveal zones potentially contaminated by gamma-emitting radionuclides. Based on interpretations by the geophysical logging contractor (Stoller, Inc.), there were no man-made gammaemitting contaminants (e.g., process uranium) detected above the minimum detectable level in these wells. The spectral gamma logs are shown on the borehole composite logs presented in the Appendix (Figures A.1 through A.4).

\subsubsection{Composite Borehole Logs}

A composite borehole log was prepared for each VOC investigation borehole. These graphics are included as separate plates in the Appendix (Figures A.1 through A.4), to facilitate reference to them while reading other sections of this report. (Note: Composite logs for the LFI boreholes drilled earlier also are included as Figures A.5 through A.8, so that all information on recent boreholes in the study area is readily available.) These interpretive logs include multiple data sets and provide a graphic, easy-to-use compilation of pertinent data and a hydrogeologic profile representing each borehole. Stratigraphic contacts, key lithologic intervals, and hydrogeologic units within each borehole are identified based on the interpretation of the available data. Depth-specific data used to construct the composite logs include the following:

- the completed well as-built diagram

- geologic log showing sediment types

- vertical locations for aquifer hydraulic tests and depth-discrete water samples

- concentrations of VOCs in water samples

- spectral gamma log data for naturally occurring uranium

- total gamma and neutron moisture logs

- stratigraphic interpretations and nomenclature.

Tables containing the results of aquifer tests and the values measured for field parameters associated with each groundwater sample collected also are included on the composite borehole logs figures.

\subsubsection{Subsurface Characterization}

The entire uppermost unconfined aquifer system was characterized in detail at each borehole to delineate the distribution of VOC contamination throughout the unconfined aquifer. The variable thickness of the permeable Hanford formation, which disconformably overlies the older and less permeable Ringold Formation sediments, was readily delineated at all four borehole locations. The distinct lithologic contrast across the Hanford-Ringold erosional contact was documented and verified using sediment samples and geophysical borehole logs.

The lower boundary for the unconfined aquifer, (i.e., top of the Ringold Formation lower mud unit) also was delineated, and sediment grab samples were collected at the base of the Ringold Formation 
gravels near the contact with the underlying lower mud. The uppermost unconfined aquifer system, defined as the saturated interval from the water table to the top of the Ringold Formation lower mud, ranges in thickness from approximately 27 meters ( 89 feet) to 29 meters (95 feet), depending on the elevation of the water table, which continually changes because of fluctuations in the Columbia River stage. Drilling did not continue deeper than the uppermost portion of Ringold Formation lower mud.

Lithologic descriptions, hydraulic test results, groundwater analytical results, and geophysical logs were used to differentiate preferential flow paths within the unconfined aquifer. The three primary hydrologic units or flow zones within the unconfined aquifer, as identified during the earlier LFI investigation and confirmed by the VOC investigation, are, in order of increasing depth:

1. Hanford formation gravel-dominated lithofacies (highly transmissive, uppermost hydrologic unit)

2. Ringold Formation Unit E interval consisting of silt and sand lithofacies (low to moderate transmissivity)

3. Ringold Formation Unit E lithofacies consisting of silty, sandy, gravelly sediment in the lower portion of the unconfined aquifer (moderately transmissive sediment). The bottom of the unconfined aquifer is at the contact between the base of Ringold Formation gravelly sequence and the underlying aquitard, that is, the Ringold Formation lower mud unit.

Combining the sediment descriptions with the depth-discrete aquifer testing results facilitated the subdivision of the aquifer into mapable hydrogeologic units based on varying hydraulic properties. The hydraulic conductivity $\left(\mathrm{K}_{\mathrm{h}}\right)$ data and the well development information confirmed that the Hanford formation gravel-dominated facies is significantly more permeable than the underlying, older Ringold Formation sediments. The Ringold Formation sediments are more compacted, variably cemented, and geochemically altered, resulting in a lower overall permeability. Measured hydraulic conductivity $\left(\mathrm{K}_{\mathrm{h}}\right)$ for the Hanford formation may exceed 300 meters per day, compared to much lower values in the underlying Ringold Formation. The Hanford-Ringold contact, which lies below the water table in most of the 300 Area, reflects an erosional paleo-channel created by Pleistocene ice age catastrophic flooding across the area (Newcomb et al. 1972; Lindberg and Bond 1979; Swanson 1992; Lindsey 1995).

The contrast in permeability across the Hanford-Ringold contact creates an effective vertical flow boundary for groundwater. Where saturated, the more permeable Hanford formation gravel-dominated facies, deposited directly onto the eroded Ringold surface, creates a preferential groundwater flow path within the very uppermost portion of the unconfined aquifer system. Characterization data used to define the contact include changes noted by the driller and in the well-site geologist borehole log, sediment grab and core sample descriptions, the borehole geophysical logs, and the integrated depth-discrete aquifer hydraulic testing and groundwater sample results.

These new data have been correlated and used to update the structure contour map for the erosional contact surface between the Hanford and Ringold formations beneath the 300 Area (Figure 2.10). The structure contour map also indicates where the finer-grained interval is present at this erosional surface (shown by tan shading) and the locations of the characterization boreholes drilled as part of the LFI and this VOC investigation. This updated map illustrates the fairly dramatic relief eroded into the Ringold Formation. The prominent northwest-to-southeast channel eroded into the older, more consolidated Ringold Formation sediment is filled with younger, less consolidated Hanford formation graveldominated sediment. The latter highly permeable channel deposits provide preferential pathways for groundwater to migrate relatively rapidly toward locations of ultimate discharge to the Columbia River. 
The paleochannel and other features of the subsurface are conceptually illustrated using the new borehole data in hydrogeologic cross sections (see Figures 2.2 through 2.4).

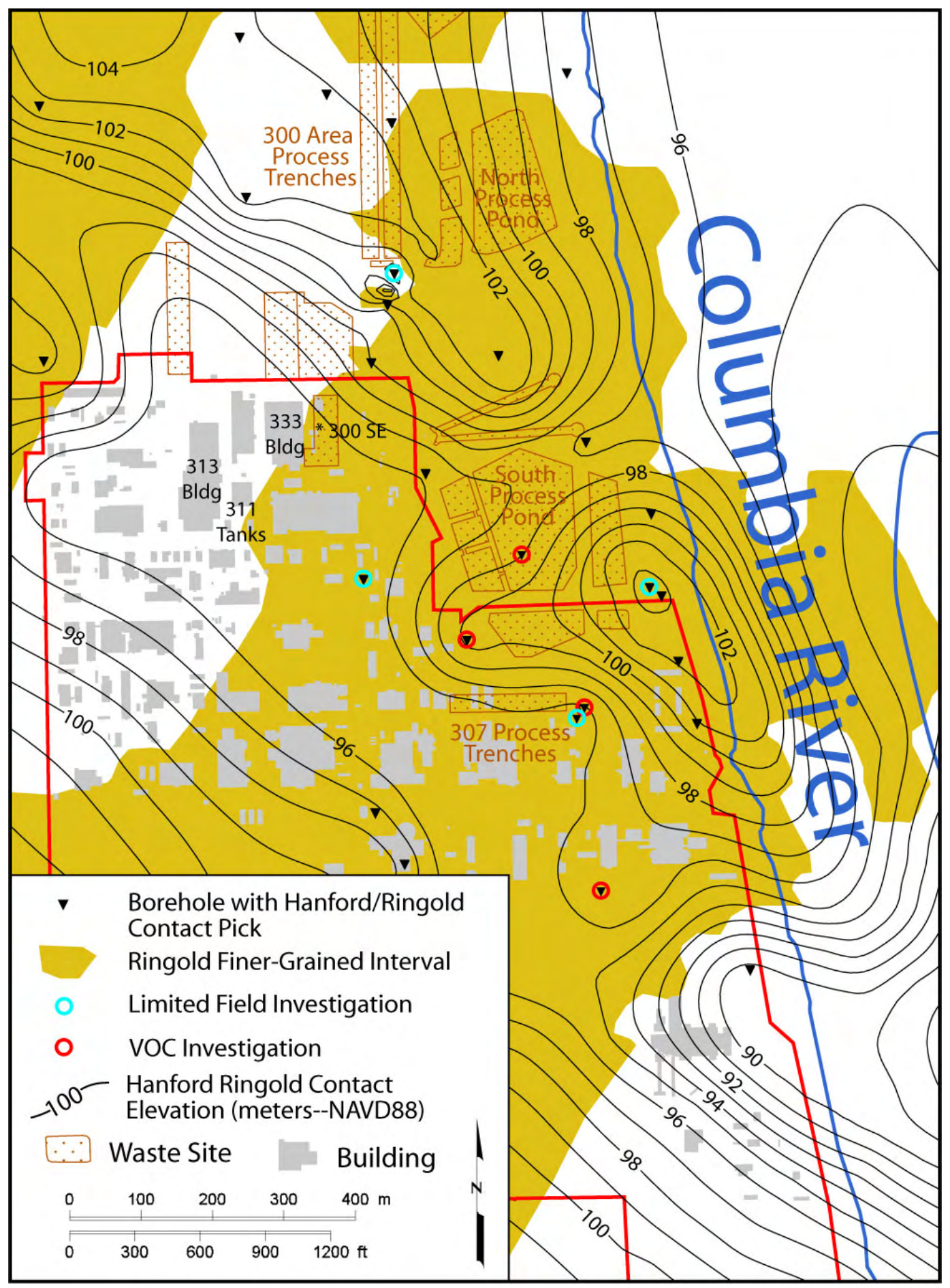

Figure 2.10. Elevation of the Contact Surface Between the Hanford and Ringold Formations Beneath the 300 Area. Tan coloring shows the area in which finer-grained Ringold sediment is present at the contact. 
The lateral continuity of the relatively finer-grained lithofacies interval in the Ringold Formation also is illustrated on the map in Figure 2.10. Explanations for those areas where the interval is not observed in boreholes include the possibility of laterally discontinuous lithofacies and/or removal by erosion. However, the relatively finer-grained interval appears to be continuous across the area east and south of the former South Process Pond, and east of the former 307 Process Trenches, where elevated concentrations of TCE and PCE have been observed in boreholes and aquifer tubes. South of the 307 Process Trenches, at borehole 399-4-14, the finer-grained interval consists of well-sorted, fine- to coarse-grained felsic-dominated sand, with interbedded matrix-supported sandy gravel. The texture and mineralogy of the sand layers, along with the sand matrix of the sandy gravel interbeds in borehole 399-4-14, are consistent throughout the finer-grained intervals. The sand layers are analogous to the lower, coarsergrained portions of the finer-grained intervals in the other three VOC investigation boreholes. The interbedded deposits encountered in borehole 399-4-14 likely represent Ringold-age sediment deposited along an active channel margin during intermittent river stages and/or sediment reworked prior to the onset of Pleistocene-age cataclysmic flooding events.

\subsection{VOC Concentrations at Shoreline Aquifer Tube Sites}

During the period of borehole drilling as part of the VOC investigation, additional monitoring along the Columbia River shoreline was conducted by sampling existing and newly installed aquifer tubes, including a special event on March 24, 2008, that involved sampling aquifer tubes and the Columbia River. Figure 2.11 is a cross section that illustrates the position of aquifer tube screens in the unconfined aquifer along the 300 Area shoreline (graphics in blue) and also the relative positions of screens in nearriver wells (graphics in gray), which have been projected onto the cross section. The contact between the Hanford formation gravels and underlying Ringold Formation is illustrated by projecting the contact, as revealed in the near-river well geology logs, onto the cross section.

At least one tube clearly is installed in the finer-grained interval in the Ringold Formation (deepest tube at site AT-3-3); several other tubes may be very near to, or within, the upper portion of that interval. During the installation of tubes, the temporary drive casing often extended into the interval, but the tube would not yield water and was therefore pulled back to a shallower depth, which would position it in the more transmissive Hanford formation gravels. The TCE concentrations for recent results are included on the cross section for samples collected from tubes and near-river wells. All historical analytical results for VOCs in samples from aquifer tubes are tabulated in Table 2.5.

The VOC concentrations in samples from tube AT-3-3-D are comparable to those observed at LFI borehole 399-3-20 (i.e., up to $~ 600 \mu \mathrm{g} / \mathrm{L}$ TCE), with lesser amounts of PCE and the degradation product cis-1,2-DCE. Much lower but still clearly detectable amounts of TCE are found in the mid-depth tube at that location (AT-3-3-M), possibly indicating that TCE is slowly releasing from the finer-grained Ringold interval into the overlying saturated Hanford gravel. A similar situation may exist at tube site AT-3-4, located approximately 200 meters downstream from AT-3-3. An alternative explanation is that the TCE observed in the shallow tube samples is associated with the mapped groundwater plume (see Figure 1.1). 
Trichloroethene in Aquifer Along the 300 Area Shoreline

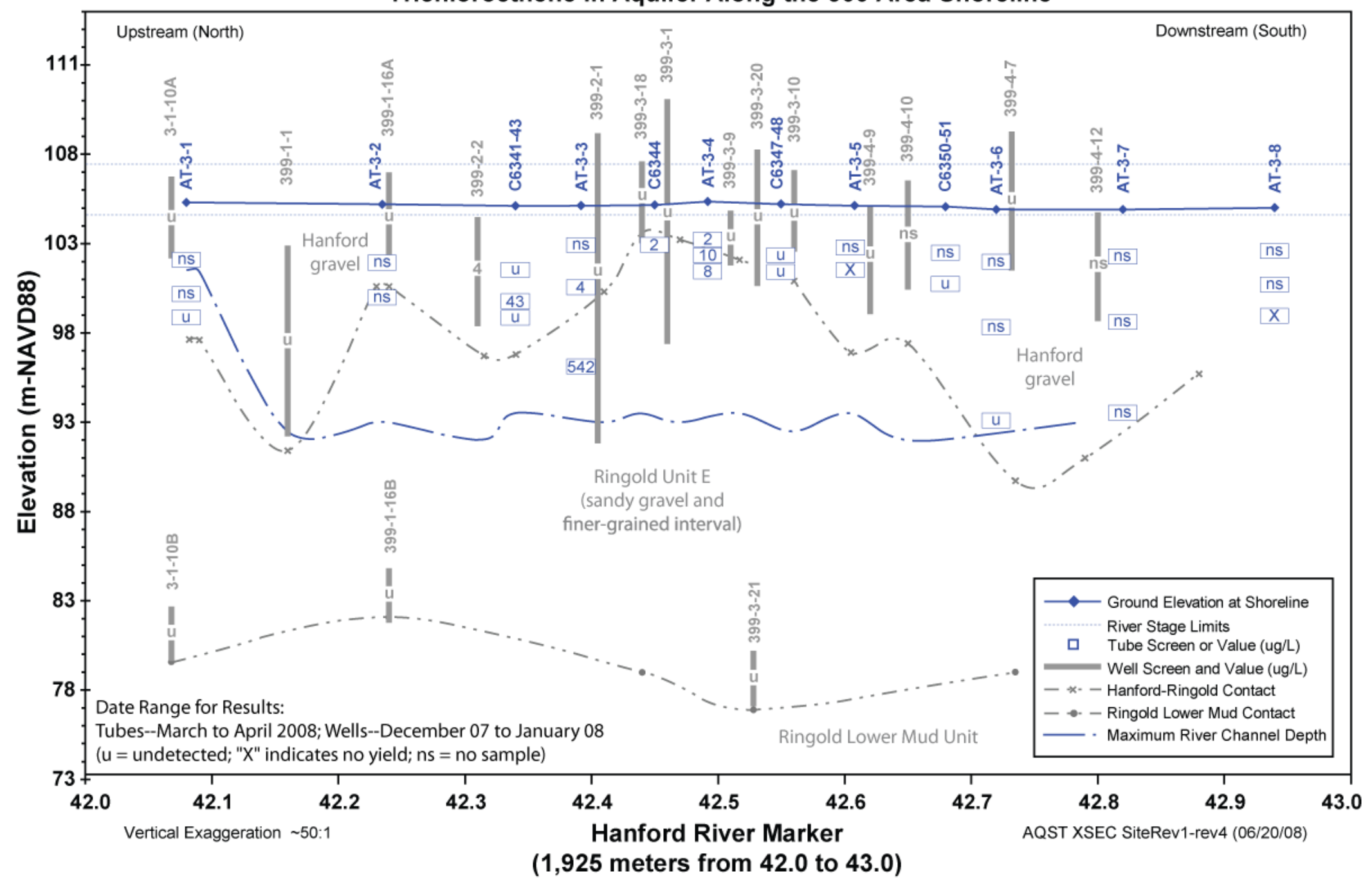

Figure 2.11. Cross Section North to South Along the 300 Area Shoreline. Recent results for trichloroethene in groundwater samples from aquifer tubes and near-river wells are shown. 
Table 2.5. Volatile Organic Compounds in Samples from Aquifer Tubes

\begin{tabular}{|c|c|c|c|c|c|c|c|c|c|}
\hline $\begin{array}{c}\text { Aquifer Tube } \\
\text { Name }\end{array}$ & $\begin{array}{l}\text { Screen } \\
\text { Depth } \\
\text { (m-below } \\
\text { ground } \\
\text { surface) }\end{array}$ & $\begin{array}{c}\text { Sample } \\
\text { Elevation } \\
\text { (m-NAVD88) }\end{array}$ & $\begin{array}{l}\text { Hydro- } \\
\text { logic } \\
\text { Unit }^{(1)}\end{array}$ & $\begin{array}{c}\text { Sample Collect } \\
\text { Date }\end{array}$ & $\begin{array}{c}\text { Specific } \\
\text { Conduc- } \\
\text { tance }{ }^{(2)} \\
(\mu \mathrm{S} / \mathrm{cm})\end{array}$ & $\begin{array}{c}\text { Trichloro- } \\
\text { ethene } \\
\text { ( } \mu \mathrm{g} / \mathrm{L}) \\
\mathrm{MCL}=5 \\
\mathrm{MDL}=0.20\end{array}$ & $\begin{array}{c}\text { Tetrachloro- } \\
\text { ethene } \\
\text { ( } \mu \mathrm{g} / \mathrm{L}) \\
\mathrm{MCL}=5 \\
\mathrm{MDL}=0.19\end{array}$ & $\begin{array}{c}\text { Cis-1,2- } \\
\text { Dichloro- } \\
\text { ethene } \\
(\mu \mathrm{g} / \mathrm{L}) \\
\mathrm{MCL}=70 \\
\mathrm{MDL}=0.19\end{array}$ & $\begin{array}{c}\text { Vinyl Chloride } \\
(\mu g / L) \\
M C L=2 \\
M D L=0.23\end{array}$ \\
\hline \multicolumn{10}{|c|}{ AT-3-1 Downgradient of 300 Area Process Trenches (316-5) and 618-5 Burial Ground } \\
\hline AT-3-1-S & 3.5 & 102.0 & $\mathrm{H}_{\text {gravel }}$ & & & & & & \\
\hline AT-3-1-M & 5.1 & 100.1 & $\mathrm{H}_{\text {gravel }}$ & $12 / 11 / 2006$ & 366 & $U$ & $U$ & $U^{(3)}$ & $\mathrm{U}$ \\
\hline AT-3-1-D(1) & 6.4 & 98.8 & $\mathrm{H}_{\text {gravel }}$ & $3 / 18 / 2004$ & 444 & U & U & $U$ & $U$ \\
\hline AT-3-1-D(1) & 6.4 & 98.8 & $\mathrm{H}_{\text {gravel }}$ & $2 / 2 / 2005$ & 380 & $\mathrm{U}$ & $U$ & $U$ & $U$ \\
\hline AT-3-1-D(1) & 6.4 & 98.8 & $\mathrm{H}_{\text {gravel }}$ & $9 / 15 / 2005$ & 439 & 0.2 & $U$ & $U$ & $U$ \\
\hline AT-3-1-D(1) & 6.4 & 98.8 & $\mathrm{H}_{\text {gravel }}$ & $1 / 24 / 2006$ & 380 & $U$ & $U$ & $U$ & $U$ \\
\hline AT-3-1-D(1) & 6.4 & 98.8 & $\mathrm{H}_{\text {gravel }}$ & $12 / 11 / 2006$ & 401 & $U$ & $U$ & $U^{(3)}$ & U \\
\hline AT-3-1-D(1) & 6.4 & 98.8 & $\mathrm{H}_{\text {gravel }}$ & $8 / 21 / 2007$ & 451 & $U$ & $U$ & $U^{(3)}$ & $U$ \\
\hline AT-3-1-D(1) & 6.4 & 98.8 & $\mathrm{H}_{\text {gravel }}$ & $11 / 2 / 2007$ & 463 & U & U & U & U \\
\hline AT-3-1-D(1) & 6.4 & 98.8 & $\mathrm{H}_{\text {gravel }}$ & $3 / 17 / 2008$ & nd & $U$ & $U$ & $U$ & $U$ \\
\hline \multicolumn{10}{|c|}{ AT-3-2 Downgradient of 300 Area Process Trenches (316-5) and North Process Pond (316-2) } \\
\hline AT-3-2-S & 3.3 & 101.9 & $\mathrm{H}_{\text {gravel }}$ & $12 / 11 / 2006$ & 197 & $\mathrm{U}$ & $\mathrm{U}$ & $U^{(3)}$ & U \\
\hline AT-3-2-M & 5.1 & 100.1 & $\mathrm{R}_{\text {unit? }}$ & $3 / 18 / 2004$ & 363 & 0.3 & U & U & U \\
\hline AT-3-2-M & 5.1 & 100.1 & $\mathrm{R}_{\text {unit? }}$ & $2 / 2 / 2005$ & 189 & 0.3 & $U$ & $U$ & $U$ \\
\hline AT-3-2-M & 5.1 & 100.1 & $\mathrm{R}_{\text {unit? }}$ & $1 / 24 / 2006$ & 193 & 0.3 & $U$ & U & $U$ \\
\hline AT-3-2-M & 5.1 & 100.1 & $\mathrm{R}_{\text {unit? }}$ & $12 / 11 / 2006$ & nd & $U$ & $U$ & $U^{(3)}$ & $U$ \\
\hline AT-3-2-M & 5.1 & 100.1 & $\mathrm{R}_{\text {unit? }}$ & $11 / 2 / 2007$ & 412 & $U$ & $U$ & U & U \\
\hline C6341-S & 3.6 & 101.5 & $\mathrm{H}_{\text {gravel }}$ & $4 / 3 / 2008$ & 301 & $U$ & $U$ & nd & $U$ \\
\hline C6342-M & 5.3 & 99.8 & $\mathrm{H}_{\text {gravel }}$ & $4 / 3 / 2008$ & 220 & 43.00 & U & nd & $U$ \\
\hline C6343-D & 6.3 & 98.8 & $\mathrm{H}_{\text {gravel }}$ & $4 / 3 / 2008$ & 183 & U & U & nd & $U$ \\
\hline \multicolumn{10}{|c|}{ AT-3-3 Downgradient of 300 Area Process Trenches (316-5), South Process Pond (316-1), and Sanitary Leach Trenches } \\
\hline AT-3-3-S & 2.1 & 102.9 & $\mathrm{H}_{\text {gravel }}$ & $12 / 11 / 2006$ & 162 & $\mathrm{U}$ & $\mathrm{U}$ & $\mathrm{U}^{(3)}$ & $\mathrm{U}$ \\
\hline AT-3-3-S & 2.1 & 102.9 & $\mathrm{H}_{\text {gravel }}$ & $11 / 2 / 2007$ & 422 & U & U & U & $U$ \\
\hline AT-3-3-M & 4.6 & 100.5 & $\mathrm{H}_{\text {gravel }}$ & $3 / 18 / 2004$ & 450 & 6.8 & $U$ & 0.2 & $U$ \\
\hline AT-3-3-M & 4.6 & 100.5 & $\mathrm{H}_{\text {gravel }}$ & 2/2/2005 & 331 & 2.9 & U & U & U \\
\hline AT-3-3-M & 4.6 & 100.5 & $\mathrm{H}_{\text {gravel }}$ & $9 / 15 / 2005$ & 431 & 1.8 & $U$ & 0.3 & $U$ \\
\hline AT-3-3-M & 4.6 & 100.5 & $\mathrm{H}_{\text {gravel }}$ & $1 / 24 / 2006$ & 354 & 1.2 & $U$ & $U$ & $U$ \\
\hline AT-3-3-M & 4.6 & 100.5 & $\mathrm{H}_{\text {gravel }}$ & $12 / 11 / 2006$ & 272 & $U$ & $U$ & $U^{(3)}$ & U \\
\hline AT-3-3-M & 4.6 & 100.5 & $\mathrm{H}_{\text {gravel }}$ & $12 / 11 / 2006$ & 272 & $U$ & $U$ & $\mathrm{U}^{(3)}$ & $U$ \\
\hline AT-3-3-M & 4.6 & 100.5 & $\mathrm{H}_{\text {gravel }}$ & $8 / 21 / 2007$ & 519 & 4.2 & $U$ & $U^{(3)}$ & $U$ \\
\hline AT-3-3-M & 4.6 & 100.5 & $\mathrm{H}_{\text {gravel }}$ & $11 / 2 / 2007$ & 475 & U & U & $U$ & U \\
\hline AT-3-3-M & 4.6 & 100.5 & $\mathrm{H}_{\text {gravel }}$ & $3 / 17 / 2008$ & nd & 4.0 & $U$ & $U$ & $U$ \\
\hline AT-3-3-D & 8.9 & 96.1 & $\mathrm{R}_{\text {sand }}$ & $9 / 5 / 2006$ & 186 & 96.0 & 1.1 & 2.0 & U \\
\hline AT-3-3-D & 8.9 & 96.1 & $\mathrm{R}_{\text {sand }}$ & $8 / 30 / 2007$ & 285 & 290.0 & 1.3 & 2.9 & $U$ \\
\hline AT-3-3-D & 8.9 & 96.1 & $\mathrm{R}_{\text {sand }}$ & $11 / 2 / 2007$ & 299 & 450.0 & 4.5 & 4.1 & $U$ \\
\hline AT-3-3-D & 8.9 & 96.1 & $\mathrm{R}_{\text {sand }}$ & $3 / 17 / 2008$ & nd & 530.0 & 4.7 & 4.8 & $U$ \\
\hline AT-3-3-D & 8.9 & 96.1 & $\mathrm{R}_{\text {sand }}$ & $3 / 24 / 2008$ & 300 & 542.0 & 5.7 & 8.5 & $U$ \\
\hline C6344-S & 2.2 & 102.9 & $\mathrm{H}_{\text {gravel }}$ & $3 / 24 / 2008$ & 431 & 1.5 & $U$ & $U$ & U \\
\hline C6344-S & 2.2 & 102.9 & $\mathrm{H}_{\text {gravel }}$ & $4 / 3 / 2008$ & 419 & $U$ & $U$ & nd & U \\
\hline
\end{tabular}


Table 2.5. (contd)

\begin{tabular}{|c|c|c|c|c|c|c|c|c|c|}
\hline $\begin{array}{l}\text { Aquifer Tube } \\
\text { Name }\end{array}$ & $\begin{array}{c}\text { Screen } \\
\text { Depth } \\
\text { (m-below } \\
\text { ground } \\
\text { surface) }\end{array}$ & $\begin{array}{c}\text { Sample } \\
\text { Elevation } \\
\text { (m-NAVD88) }\end{array}$ & $\begin{array}{l}\text { Hydro- } \\
\text { logic } \\
\text { Unit }\end{array}$ & $\begin{array}{c}\text { Sample Collect } \\
\text { Date }\end{array}$ & $\begin{array}{l}\text { Specific } \\
\text { Conduc- } \\
\text { tance }{ }^{(2)} \\
(\mu \mathrm{S} / \mathrm{cm})\end{array}$ & $\begin{array}{c}\text { Trichloro- } \\
\text { ethene } \\
(\mu \mathrm{g} / \mathrm{L}) \\
M C L=5 \\
M D L=0.20\end{array}$ & $\begin{array}{c}\text { Tetrachloro- } \\
\text { ethene } \\
(\mu \mathrm{g} / \mathrm{L}) \\
\mathrm{MCL}=5 \\
M D L=0.19\end{array}$ & $\begin{array}{c}\text { Cis-1,2- } \\
\text { Dichloro- } \\
\text { ethene } \\
(\mu \mathrm{g} / \mathrm{L}) \\
\mathrm{MCL}=70 \\
\mathrm{MDL}=0.19\end{array}$ & $\begin{array}{c}\text { Vinyl Chloride } \\
(\mu g / L) \\
M C L=2 \\
M D L=0.23\end{array}$ \\
\hline \multicolumn{10}{|c|}{ AT-3-4 Downgradient of South Process Pond (316-1) } \\
\hline AT-3-4-S & 2.1 & 103.2 & $\mathrm{R}_{\text {sand? }}$ & $3 / 18 / 2004$ & 439 & 0.7 & $\mathrm{U}$ & $\mathrm{U}$ & $\mathrm{U}$ \\
\hline AT-3-4-S & 2.1 & 103.2 & $\mathrm{R}_{\text {sand? }}$ & $2 / 2 / 2005$ & 276 & 0.7 & U & $U$ & U \\
\hline AT-3-4-S & 2.1 & 103.2 & $\mathrm{R}_{\text {sand? }}$ & 9/15/2005 & 381 & 1.2 & $\mathrm{U}$ & $\mathrm{U}$ & $\mathrm{U}$ \\
\hline AT-3-4-S & 2.1 & 103.2 & $\mathrm{R}_{\text {sand? }}$ & $1 / 24 / 2006$ & 229 & 0.4 & $\mathrm{U}$ & u & $\mathrm{U}$ \\
\hline AT-3-4-S & 2.1 & 103.2 & $\mathrm{R}_{\text {sand? }}$ & $12 / 12 / 2006$ & 203 & u & $\mathrm{U}$ & $\mathrm{U}^{(3)}$ & $\mathrm{U}$ \\
\hline AT-3-4-S & 2.1 & 103.2 & $\mathrm{R}_{\text {sand? }}$ & $8 / 22 / 2007$ & 391 & 1.5 & $U$ & $\mathrm{U}^{(3)}$ & $U$ \\
\hline AT-3-4-S & 2.1 & 103.2 & $\mathrm{R}_{\text {sand? }}$ & $11 / 5 / 2007$ & 357 & $U$ & $U$ & $U$ & $U$ \\
\hline AT-3-4-S & 2.1 & 103.2 & $\mathrm{R}_{\text {sand? }}$ & $3 / 18 / 2008$ & 382 & 1.5 & U & U & U \\
\hline AT-3-4-M & 2.8 & 102.5 & $\mathrm{R}_{\text {sand? }}$ & $12 / 12 / 2006$ & 276 & 28.0 & $U$ & 2.5 & $U$ \\
\hline AT-3-4-M & 2.8 & 102.5 & $\mathrm{R}_{\text {sand? }}$ & $11 / 5 / 2007$ & 383 & 8.0 & U & $U$ & $U$ \\
\hline AT-3-4-M & 2.8 & 102.5 & $\mathrm{R}_{\text {sand? }}$ & $3 / 18 / 2008$ & 277 & 10.0 & $U$ & $U$ & U \\
\hline AT-3-4-D & 3.7 & 101.6 & $\mathrm{R}_{\text {sand? }}$ & $9 / 5 / 2006$ & 373 & 5.1 & U & 0.3 & U \\
\hline AT-3-4-D & 3.7 & 101.6 & $\mathrm{R}_{\text {sand? }}$ & $12 / 12 / 2006$ & 201 & 9.3 & $U$ & $U^{(3)}$ & U \\
\hline AT-3-4-D & 3.7 & 101.6 & $\mathrm{R}_{\text {sand? }}$ & $8 / 30 / 2007$ & 318 & 4.0 & $U$ & $\mathrm{U}^{(3)}$ & U \\
\hline AT-3-4-D & 3.7 & 101.6 & $\mathrm{R}_{\text {sand? }}$ & $11 / 5 / 2007$ & 385 & 3.8 & $U$ & $U$ & U \\
\hline AT-3-4-D & 3.7 & 101.6 & $\mathrm{R}_{\text {sand? }}$ & $3 / 18 / 2008$ & 358 & 5.7 & U & U & U \\
\hline AT-3-4-D & 3.7 & 101.6 & $\mathrm{R}_{\text {sand? }}$ & $3 / 24 / 2008$ & 405 & 7.8 & $U$ & U & $U$ \\
\hline C6347-M & 3.0 & 102.2 & $\mathrm{R}_{\text {sand? }}$ & $4 / 7 / 2008$ & 268 & $U$ & $U$ & nd & $U$ \\
\hline C6348-D & 3.7 & 101.5 & $\mathrm{R}_{\text {sand? }}$ & $4 / 7 / 2008$ & 273 & U & U & nd & U \\
\hline \multicolumn{10}{|c|}{ AT-3-5 Downgradient of South Process Pond (316-1) and 307 Process Trenches (316-3) } \\
\hline AT-3-5-S & 2.3 & 102.8 & $\mathrm{H}_{\text {gravel }}$ & $3 / 18 / 2004$ & 271 & 0.5 & $\mathrm{U}$ & $\mathrm{U}$ & $\mathrm{U}$ \\
\hline AT-3-5-S & 2.3 & 102.8 & $\mathrm{H}_{\text {gravel }}$ & $2 / 2 / 2005$ & 175 & $\mathrm{U}$ & 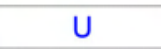 & $u$ & $u$ \\
\hline AT-3-5-S & 2.3 & 102.8 & $\mathrm{H}_{\text {gravel }}$ & $1 / 24 / 2006$ & 233 & 0.4 & $u$ & $u$ & $u$ \\
\hline AT-3-5-S & 2.3 & 102.8 & $\mathrm{H}_{\text {gravel }}$ & $9 / 5 / 2006$ & 297 & 0.8 & $u$ & $U$ & $u$ \\
\hline AT-3-5-S & 2.3 & 102.8 & $\mathrm{H}_{\text {gravel }}$ & $12 / 12 / 2006$ & 206 & $u$ & U & $\mathrm{U}^{(3)}$ & U \\
\hline AT-3-5-S & 2.3 & 102.8 & $\mathrm{H}_{\text {gravel }}$ & $11 / 5 / 2007$ & 372 & $u$ & $u$ & $U$ & $u$ \\
\hline AT-3-5-M & 3.7 & 101.5 & $\mathrm{H}_{\text {gravel }}$ & & & & & & \\
\hline C6350-S & 2.6 & 102.5 & $\mathrm{H}_{\text {gravel }}$ & $4 / 7 / 2008$ & 222 & na & na & na & na \\
\hline C6351-M & 4.3 & 100.7 & $\mathrm{H}_{\text {gravel }}$ & $3 / 24 / 2008$ & 401 & 0.87 & $U$ & $U$ & U \\
\hline C6351-M & 4.3 & 100.7 & $\mathrm{H}_{\text {gravel }}$ & $4 / 7 / 2008$ & 222 & na & na & na & na \\
\hline \multicolumn{10}{|c|}{ AT-3-6 Within Major Paleochannel } \\
\hline AT-3-6-S & 2.9 & 102.0 & $\mathrm{H}_{\text {gravel }}$ & $3 / 18 / 2004$ & 423 & 1.3 & $U$ & $U$ & $U$ \\
\hline AT-3-6-S & 2.9 & 102.0 & $\mathrm{H}_{\text {gravel }}$ & $2 / 2 / 2005$ & 326 & 0.5 & $U$ & $\mathrm{U}$ & $U$ \\
\hline AT-3-6-S & 2.9 & 102.0 & $\mathrm{H}_{\text {gravel }}$ & $12 / 14 / 2006$ & 175 & $\mathrm{U}$ & $U$ & $\mathrm{U}^{(3)}$ & $U$ \\
\hline AT-3-6-S & 2.9 & 102.0 & $\mathrm{H}_{\text {gravel }}$ & $11 / 5 / 2007$ & 354 & $U$ & U & $U$ & U \\
\hline AT-3-6-M & 6.7 & 98.3 & $\mathrm{H}_{\text {gravel }}$ & $12 / 14 / 2006$ & 410 & U & U & $U^{(3)}$ & U \\
\hline AT-3-6-D & 11.8 & 93.1 & $R_{\text {unit? }}$ & $12 / 14 / 2006$ & 227 & U & U & $U^{(3)}$ & U \\
\hline AT-3-6-D & 11.8 & 93.1 & $\mathrm{R}_{\text {unit? }}$ & $11 / 5 / 2007$ & 227 & $U$ & $U$ & $U$ & $U$ \\
\hline AT-3-6-D & 11.8 & 93.1 & $\mathrm{R}_{\text {unit? }}$ & $3 / 24 / 2008$ & 219 & $U$ & $U$ & $U$ & $U$ \\
\hline
\end{tabular}


Table 2.5. (contd)

\begin{tabular}{|c|c|c|c|c|c|c|c|c|c|}
\hline $\begin{array}{c}\text { Aquifer Tube } \\
\text { Name }\end{array}$ & $\begin{array}{l}\text { Screen } \\
\text { Depth } \\
\text { (m-below } \\
\text { ground } \\
\text { surface) }\end{array}$ & $\begin{array}{c}\text { Sample } \\
\text { Elevation } \\
\text { (m-NAVD88) }\end{array}$ & $\begin{array}{l}\text { Hydro- } \\
\text { logic } \\
\text { Unit }{ }^{(1)}\end{array}$ & $\begin{array}{c}\text { Sample Collect } \\
\text { Date }\end{array}$ & $\begin{array}{l}\text { Specific } \\
\text { Conduc- } \\
\text { tance }{ }^{(2)} \\
(\mu \mathrm{S} / \mathrm{cm})\end{array}$ & $\begin{array}{l}\text { Trichloro- } \\
\text { ethene } \\
(\mu \mathrm{g} / \mathrm{L}) \\
\mathrm{MCL}=5 \\
\mathrm{MDL}=0.20\end{array}$ & $\begin{array}{l}\text { Tetrachloro- } \\
\text { ethene } \\
(\mu g / L) \\
M C L=5 \\
M D L=0.19\end{array}$ & $\begin{array}{c}\text { Cis-1,2- } \\
\text { Dichloro- } \\
\text { ethene } \\
(\mu \mathrm{g} / \mathrm{L}) \\
\mathrm{MCL}=70 \\
\mathrm{MDL}=0.19\end{array}$ & $\begin{array}{l}\text { Vinyl Chloride } \\
\quad(\mu g / L) \\
M C L=2 \\
M D L=0.23\end{array}$ \\
\hline \multicolumn{10}{|c|}{ AT-3-7 Within Major Paleochannel } \\
\hline AT-3-7-S & 2.6 & 102.3 & $\mathrm{H}_{\text {gravel }}$ & $12 / 14 / 2006$ & 172 & U & U & $U^{(3)}$ & U \\
\hline AT-3-7-M & 6.4 & 98.6 & $\mathrm{H}_{\text {gravel }}$ & $3 / 18 / 2004$ & 524 & 2.1 & U & $U$ & U \\
\hline AT-3-7-M & 6.4 & 98.6 & $\mathrm{H}_{\text {gravel }}$ & $2 / 2 / 2005$ & 429 & 1.7 & 0.3 & U & $U$ \\
\hline AT-3-7-M & 6.4 & 98.6 & $\mathrm{H}_{\text {gravel }}$ & $2 / 2 / 2005$ & 429 & 1.6 & 0.3 & $U$ & $U$ \\
\hline AT-3-7-M & 6.4 & 98.6 & $\mathrm{H}_{\text {gravel }}$ & $1 / 24 / 2006$ & 497 & 1.7 & 0.2 & $U$ & $U$ \\
\hline AT-3-7-M & 6.4 & 98.6 & $\mathrm{H}_{\text {gravel }}$ & $12 / 14 / 2006$ & 331 & $\mathrm{U}$ & $\mathrm{U}$ & $U^{(3)}$ & $U$ \\
\hline AT-3-7-M & 6.4 & 98.6 & $\mathrm{H}_{\text {gravel }}$ & $11 / 5 / 2007$ & 455 & $U$ & $U$ & $U$ & $U$ \\
\hline AT-3-7-D & 11.4 & 93.5 & $\mathrm{R}_{\text {unit? }}$ & $12 / 14 / 2006$ & 251 & 57.0 & U & $U^{(3)}$ & U \\
\hline AT-3-7-D & 11.4 & 93.5 & $\mathrm{R}_{\text {unit? }}$ & $11 / 5 / 2007$ & 372 & 96.0 & 1.8 & $U$ & $U$ \\
\hline \multicolumn{10}{|c|}{ AT-3-8 At Southern Extent of Uranium Plume } \\
\hline AT-3-8-S & 2.4 & 102.6 & $\mathrm{H}_{\text {gravel }}$ & $3 / 18 / 2004$ & 564 & 1.2 & U & $U$ & U \\
\hline AT-3-8-S & 2.4 & 102.6 & $\mathrm{H}_{\text {gravel }}$ & $2 / 2 / 2005$ & 373 & 0.7 & $U$ & U & $U$ \\
\hline AT-3-8-S & 2.4 & 102.6 & $\mathrm{H}_{\text {gravel }}$ & $1 / 24 / 2006$ & 450 & 0.7 & $U$ & $U$ & $U$ \\
\hline AT-3-8-S & 2.4 & 102.6 & $\mathrm{H}_{\text {gravel }}$ & $12 / 14 / 2006$ & 191 & U & $U$ & $U^{(3)}$ & U \\
\hline AT-3-8-S & 2.4 & 102.6 & $\mathrm{H}_{\text {gravel }}$ & $11 / 6 / 2007$ & 367 & U & U & U & U \\
\hline AT-3-8-M & 4.3 & 100.7 & $\mathrm{H}_{\text {gravel }}$ & $12 / 14 / 2006$ & 373 & $U$ & $U$ & $U^{(3)}$ & $U$ \\
\hline AT-3-8-M & 4.3 & 100.7 & $\mathrm{H}_{\text {gravel }}$ & $11 / 6 / 2007$ & 506 & $U$ & $U$ & $U$ & U \\
\hline AT-3-8-D & 6.1 & 98.9 & $\mathrm{H}_{\text {gravel }}$ & & & & & & \\
\hline \multicolumn{10}{|c|}{$\begin{array}{l}\text { Color Key: Blue = undetected }(U) ; \text { Black = detected; Red = Exceeds } \mathrm{MCL} ; \text { Gray indicates no longer in service } \\
\text { Abbreviations: } \mathrm{MCL}=\text { maximum contaminant level for drinking water; } \mathrm{MDL}=\text { method detection limit; } \mathrm{U}=\text { undetected; } \\
\text { nd = not determined; na = not analyzed }\end{array}$} \\
\hline \multicolumn{10}{|c|}{$\begin{array}{l}\text { Footnotes: }{ }^{(1)} \mathrm{H}=\text { Hanford gravels, } \mathrm{R}=\text { Ringold Unit } \mathrm{E} ;{ }^{(2)} \text { Values from HEIS, or field notes if not yet in HEIS; } \\
\text { (3) Result for 1,2-dichloroethene, total }\end{array}$} \\
\hline \multicolumn{10}{|c|}{ Ground elevation at aquifer tube sites (m-NAVD88): } \\
\hline \multicolumn{10}{|c|}{$\begin{array}{l}\text { AT-3-1 (105.273); AT-3-2 (105.279); AT-3-3 (104.956); AT-3-4 (105.299); AT-3-5 (105.198); AT-3-6 (104.873); AT-3-7 } \\
\text { (104.870); and AT-3-8 (105.049); assumed at C6341-43 (105.09); C6344 (105.15); C6347-48 (105.2); C6350-51 (105.05) }\end{array}$} \\
\hline
\end{tabular}

\subsection{VOC Contamination in the Columbia River Environment}

The lateral extent of the finer-grained interval in the Ringold Formation out into the Columbia River channel was investigated by the Remedial Action and Closure Science (RACS) project as part of that project's larger look at the hyporheic zone in the 300 Area (Fritz et al. 2007, pp. 3.1-3.11; additional results are presented in Peterson et al. 2008, pp. 4.17-4.25). The project used a variety of approaches to investigate the contact between the Hanford and Ringold sediment along the shoreline and into the river channel, including drive-pipe penetration tests, underwater video, geophysical surveys, and hydrologic tests. The investigators also projected the elevation of the Hanford-Ringold contact onto the bathymetry of the river channel, thus outlining the potential area where the 300 Area uranium plume, which is confined primarily to saturated Hanford sediment, might upwell through the riverbed (Mackley and Fritz 2007). This area is shown in red on Figure 2.12. The riverbed area not shown in red, on the Hanford Site side of the deepest part of the channel (thalweg), reveals where the river has incised into Ringold sediment. Some portion of that area may be where the finer-grained interval sediment is exposed. Core 
samples taken along the shoreline support the presumption that the finer-grained interval in the Ringold is relatively continuous in this area and likely extends out into the river channel (Thorne 2008, pp. 4.214.23).

A second rendition of the Hanford-Ringold contact beneath the 300 Area and adjacent Columbia River channel is shown in Figure 2.13. This 3-D graphic was prepared previously to help illustrate the hydrogeologic framework for uranium contamination in the 300 Area (Thorne 2008). However, the cutaway portion of the graphic, which has the contact as its surface, uses separate colors to indicate either gravelly sediment or the finer-grained interval within Ringold Formation Unit E. Well and aquifer tube locations where VOC contamination has been encountered in the finer-grained interval are noted with a black ellipse. Areas where the river channel incises more deeply than the elevation of this contact are outlined in black. The finer-grained interval is potentially exposed in an area of riverbed just to the southeast of the southeastern corner of the former South Process Pond.

During the special sampling event conducted on March 24, 2008, Columbia River water was collected from near-bottom water depths at six locations chosen to coincide with where the finer-grained unit is likely to be incised by the river channel (see Figures 2.10 and 2.12). These locations are in the channel adjacent to the area where VOC contamination was discovered in LFI and VOC investigation boreholes. The river water samples were collected from as near to the bottom as practical by pumping through a tube lowered from a boat. The river water analytical results are listed in Table 2.6; no VOCs were detected in these samples.

Table 2.6. Results for Columbia River Water Samples Collected Offshore of the South Process Pond

\begin{tabular}{|c|c|c|c|c|c|c|c|c|c|c|}
\hline $\begin{array}{c}\text { Sample Site } \\
\text { Name }\end{array}$ & $\begin{array}{c}\text { Sample Collect } \\
\text { Date } \\
\end{array}$ & $\begin{array}{l}\text { Sample } \\
\text { Number } \\
\text { (HEIS) }^{(a)} \\
\end{array}$ & $\begin{array}{c}\text { River } \\
\text { Depth } \\
(\mathrm{m})\end{array}$ & $\begin{array}{c}\text { Sample Specific } \\
\text { Conductance } \\
(\mu \mathrm{S} / \mathrm{cm})\end{array}$ & $\begin{array}{c}\text { Trichloro- } \\
\text { ethene } \\
(\mu g / L) \\
M C L=5 \\
M D L=0.20\end{array}$ & $\begin{array}{c}\text { Tetrachloro- } \\
\text { ethene } \\
(\mu g / L) \\
M C L=5 \\
M D L=0.19\end{array}$ & $\begin{array}{c}\text { Cis-1,2- } \\
\text { Dichloro- } \\
\text { ethene } \\
(\mu \mathrm{g} / \mathrm{L}) \\
\mathrm{MCL}=70 \\
\mathrm{MDL}=0.19\end{array}$ & $\begin{array}{c}\text { Vinyl } \\
\text { Chloride } \\
(\mu \mathrm{g} / \mathrm{L}) \\
\mathrm{MCL}=\mathbf{2} \\
\mathrm{MDL}=0.23\end{array}$ & $\begin{array}{c}\text { EASTING } \\
\text { (m-NAD83) }\end{array}$ & $\begin{array}{l}\text { NORTHING } \\
\text { (m-NAD83) }\end{array}$ \\
\hline VOC-R-1 & $3 / 24 / 2008$ & B1V1W5 & 10 & 144 & $U$ & $U$ & $U$ & U & 594,573 & 116,298 \\
\hline VOC-R-2 & $3 / 24 / 2008$ & B1V1W6 & 11 & 146 & $U$ & $U$ & $U$ & $U$ & 594,618 & 116,139 \\
\hline VOC-R-3 & $3 / 24 / 2008$ & B1V1W7 & 5 & 143 & U & U & U & U & 594,598 & 115,978 \\
\hline VOC-R-4 & $3 / 24 / 2008$ & B1V1W8 & 4 & 145 & $U$ & U & $U$ & $U$ & 594,601 & 115,891 \\
\hline VOC-R-5 & $3 / 24 / 2008$ & B1V1W9 & 4 & 146 & $U$ & $U$ & U & $U$ & 594,615 & 115,834 \\
\hline VOC-R-6 & $3 / 24 / 2008$ & B1V1X0 & 9 & 156 & $U$ & $U$ & U & $U$ & 594,669 & 115,788 \\
\hline
\end{tabular}

Notes: Samples collected via pumping through a tube positioned as close as practical to the riverbed. Distance from shore based on expected location where contaminated sediment interval might outcrop in river channel. Water samples are unfiltered samples.

(a) Hanford Environmental Information System; "U" indicates undetected. 


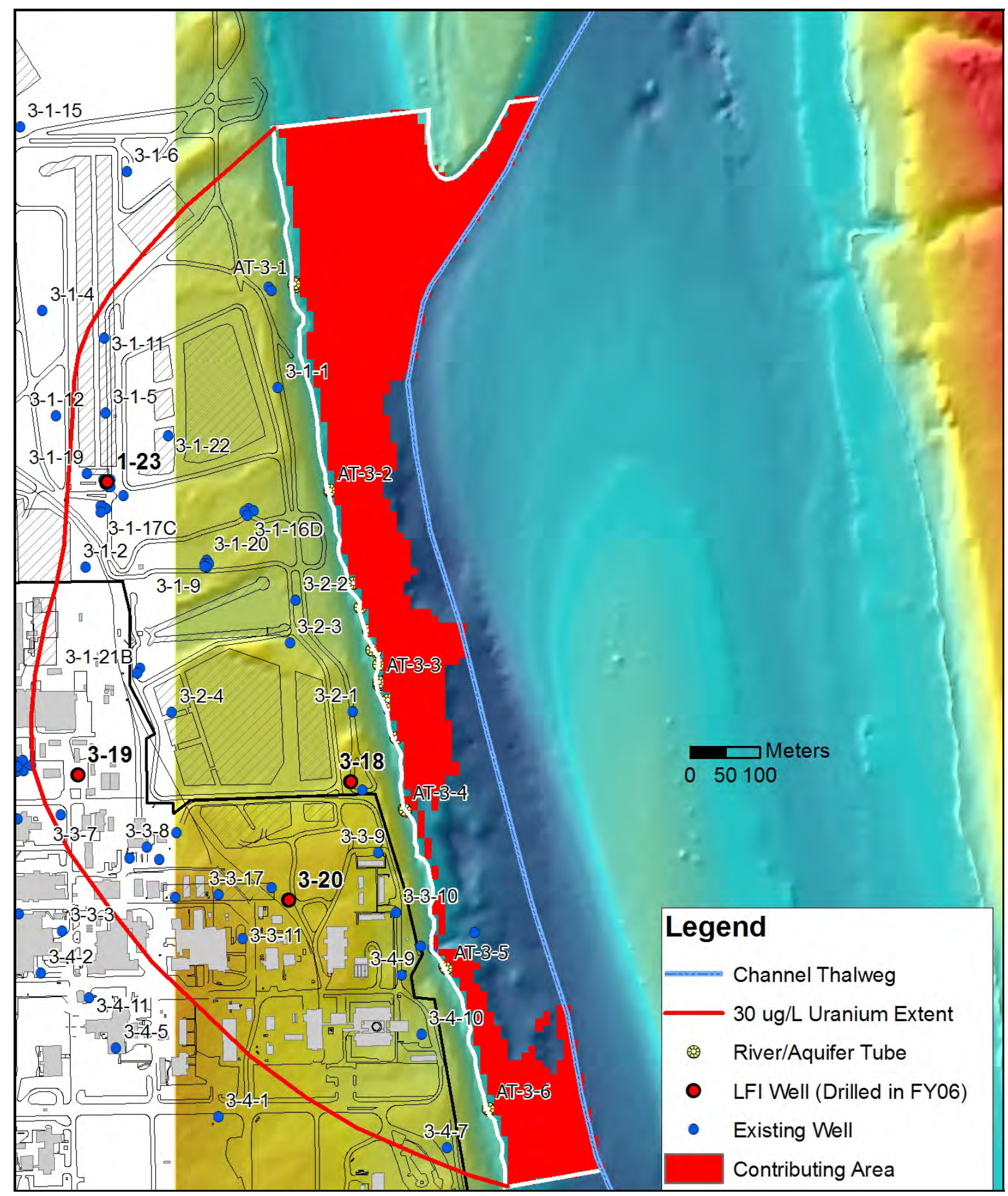

Figure 2.12. Riverbed Adjacent to 300 Area Where Saturated Hanford Formation Is Incised by the River Channel (from Mackley and Fritz 2007) 


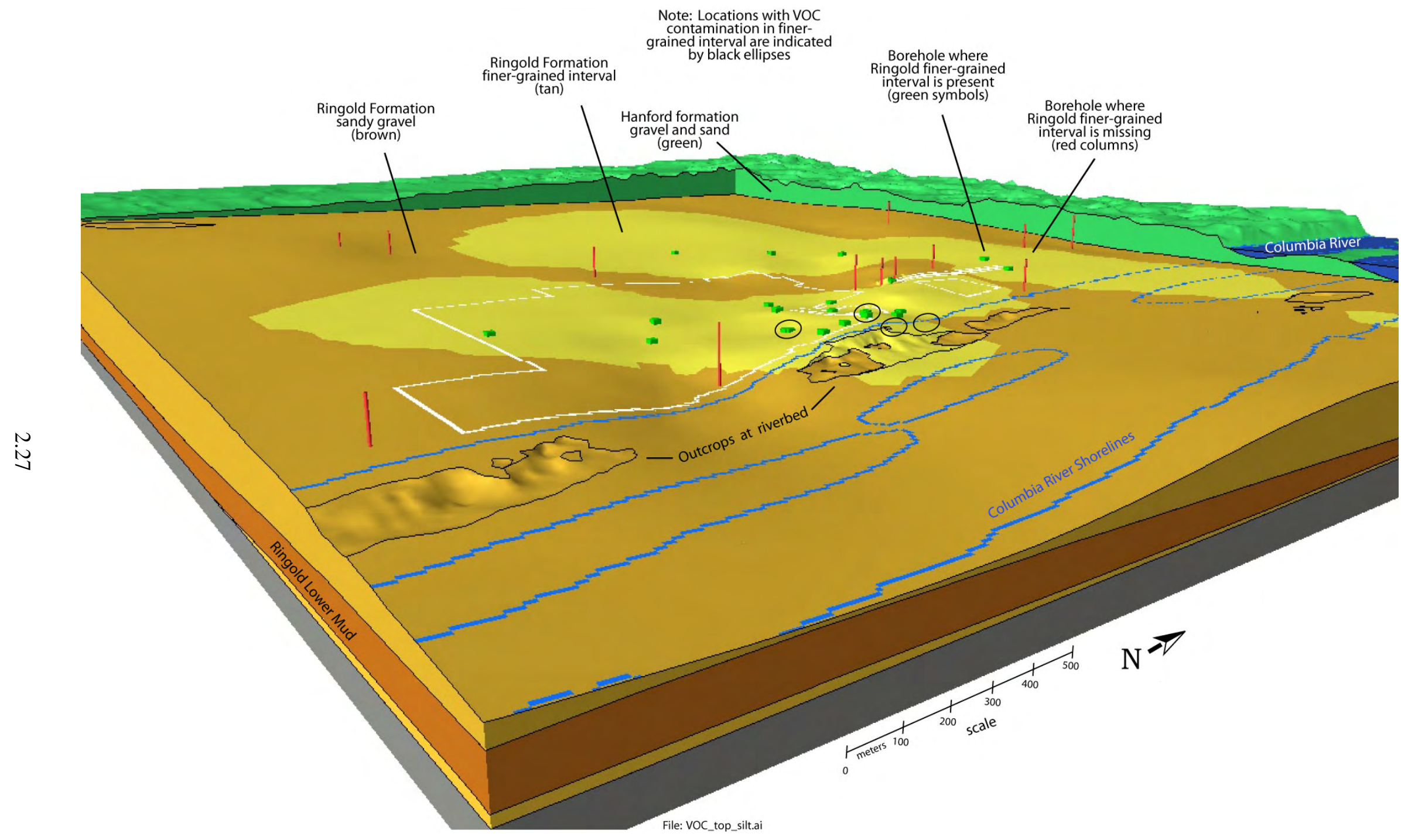

Figure 2.13. Perspective View of Contact Between Hanford and Ringold Formations Beneath the 300 Area 



\subsection{Discussion}

The following discussion focuses on three topics: potential sources for the VOC contamination encountered during the LFI and VOC investigation and possible emplacement mechanisms, exposure pathways for VOC contamination in the finer-grained interval within Ringold Formation, and the potential relationship between the occurrence investigated in this study and the presence of DCE at a monitoring well north of the current study area of investigation.

\subsection{Potential Sources for TCE, PCE, and DCE Observed in Drilling Samples}

Organic compounds such as TCE and PCE were used routinely in the 300 Area during fuels fabrication activities, with the greatest rate of production during the 1950s and 1960s. The locations of buildings where these chemicals were used, a tank farm where they were stored, and a small treatment facility are shown on previous Figure 2.10. TCE and PCE were used for vapor degreasing during the uranium core milling process and other subsequent steps in "canning” fuel (Gerber 1992). These activities took place in the 313 and 333 buildings. A 10,000-gallon storage tank was located near these buildings (a part of the "311 tank farm") and underground pipes were used to distribute the chemicals (Loe 1967; Young and Fruchter 1991). Chemicals were delivered to the storage tanks via truck. A review of unplanned releases from this tank farm does not show any that include TCE or PCE (Young and Fruchter 1991, Table 4). The used degreasing solvents most likely were disposed to the process sewer system, which discharged to the North and South process ponds until 1975. Initial remedial investigation activities at the two process ponds did not reveal evidence for VOC contamination in sediment samples from the ponds (however, they did detect polychlorinated biphenyls), but the presence in groundwater of TCE and PCE was noted (Dennison et al. 1989).

Relatively small amounts ( 5 to 10 gallons per week) of organic solutions from the 3706 building (original radiochemistry laboratory) and subsequent laboratories were disposed to a stainless steel pipe located on the dike along the north side of the South Process Pond (Clukey 1954, p. 6; Young and Fruchter 1991, p. 3.4). These organic solutions were not disposed to the process sewers because of their immiscibility and potential for creating an explosion hazard in a confined space; the specific chemicals involved are not listed in the historical reports. Other organic wastes from the 321 Separations Building that contained uranium were transported to the 316-4 cribs, located 5 miles to the northwest of the 300 Area, near the 618-10 burial ground. Tributyl phosphate and hexone are listed as common waste effluents, among with a variety of other separations processing chemicals (Gerber 1992).

Contaminated organic solvents from N-Reactor fuels fabrication were stored in the 300 Area Solvent Evaporator (WIDS code 300 SE) from 1975 to 1985 (Deford et al. 1994, pp. 3-27-3-31). This evaporator was essentially a modified dumpster and was located just to the east of the 333 Building (see Figure 2.10). Approximately 600 gallons per year of degreasing solvent, predominantly PCE, and steam condensate were disposed to this facility, according to information in WIDS. No significant spills from this facility are documented. However, there is a report describing two accidental releases of PCE to the 300 Area process trenches during this time period (Cline et al. 1985, pp. 45-49). The first occurred in November 1982 and involved 120 gallons, while the second occurred in July 1984 and involved a volume in the range 12-20 gallons. Groundwater near the trenches was monitored closely following notification of each of these releases, and the contaminant plumes created were tracked to the Columbia River. 
During the first release, the highest groundwater concentration observed near the trenches was 1,840 $\mu \mathrm{g} / \mathrm{L}$ (Figure 3.1), and a sample from a riverbank spring (S3-DR42-2 "Spring 9") revealed a PCE concentration of $270 \mu \mathrm{g} / \mathrm{L}$. During the second release, the highest concentration observed near the trenches was $691 \mu \mathrm{g} / \mathrm{L}$ (Figure 3.2), with concentrations at a riverbank spring in the range less than 1 to $20 \mu \mathrm{g} / \mathrm{L}$. By tracking the plume along the path from well 399-1-5 (at the trenches), to 399-1-3, 399-2-2, and 399-2-1 (see Figure 1.2 for locations), a travel time of approximately 35 feet (11 meters) per day was determined. This illustrates the rapid lateral transit of contamination in the saturated Hanford formation gravels to the Columbia River and also the southeastern course that groundwater follows from the former process trenches.

A second episode involving PCE in groundwater occurred during 1998 and involved increased concentrations at three wells located to the east and south of the former 300 Area Process Trenches and North Process Pond. Lindberg and Chou (2001, pp. 4.17-4.19) describe the concentration trends at wells 399-1-10A, 399-1-16A, and 399-1-17A (Figure 3.3; see Figure 1.2 for locations). The highest concentration observed was $38 \mu \mathrm{g} / \mathrm{L}$ at 399-1-17A, located near the south end of the process trenches. They were not able to positively identify the source but speculated that the high water table conditions during 1996-1997 may have remobilized contamination from earlier disposal that remained in the lower vadose zone. Based on the timing of the concentration peaks at the three wells, it appears that 399-1-17A is closest to a source.

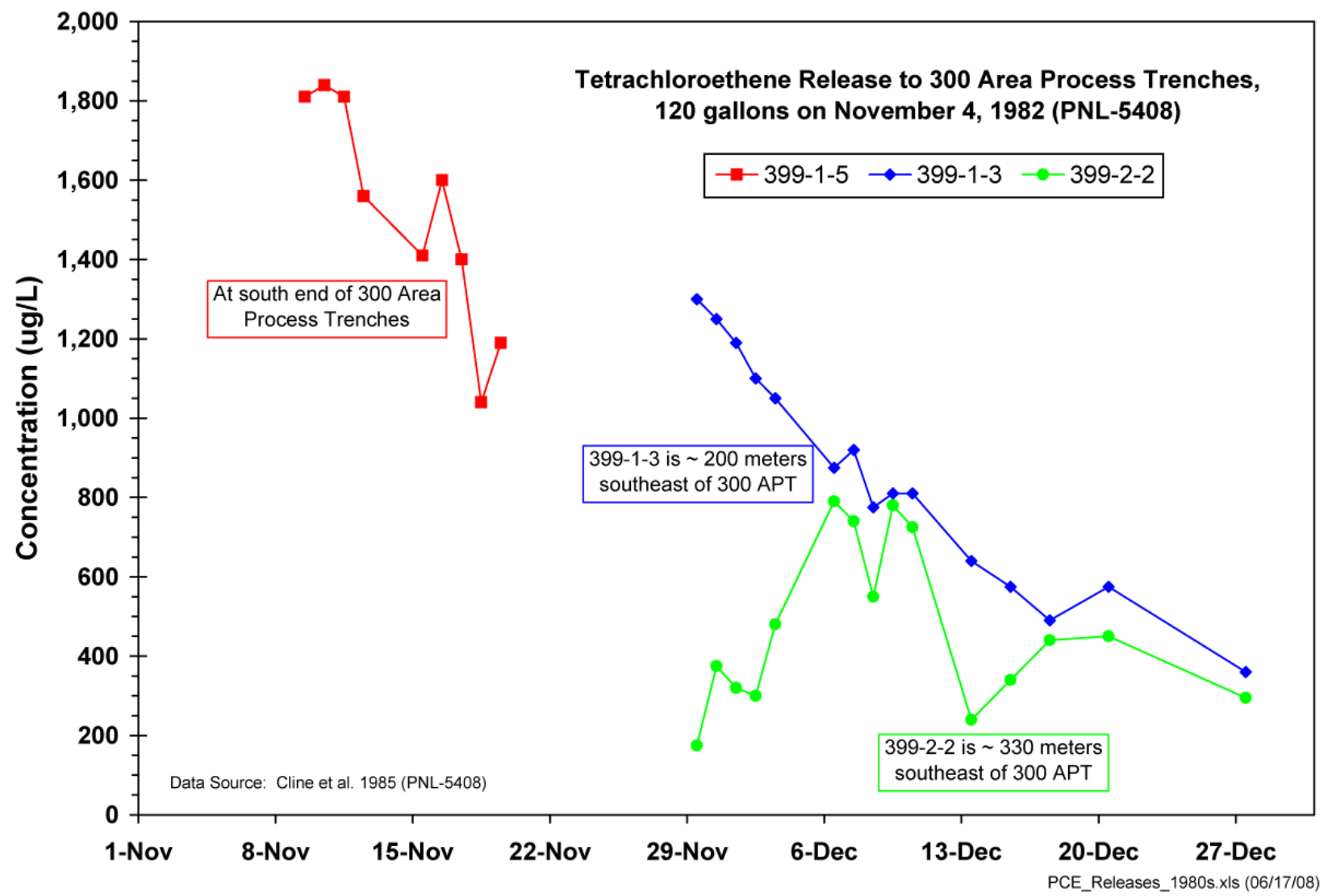

Figure 3.1. Tetrachloroethene in Groundwater from Release on November 4, 1982 


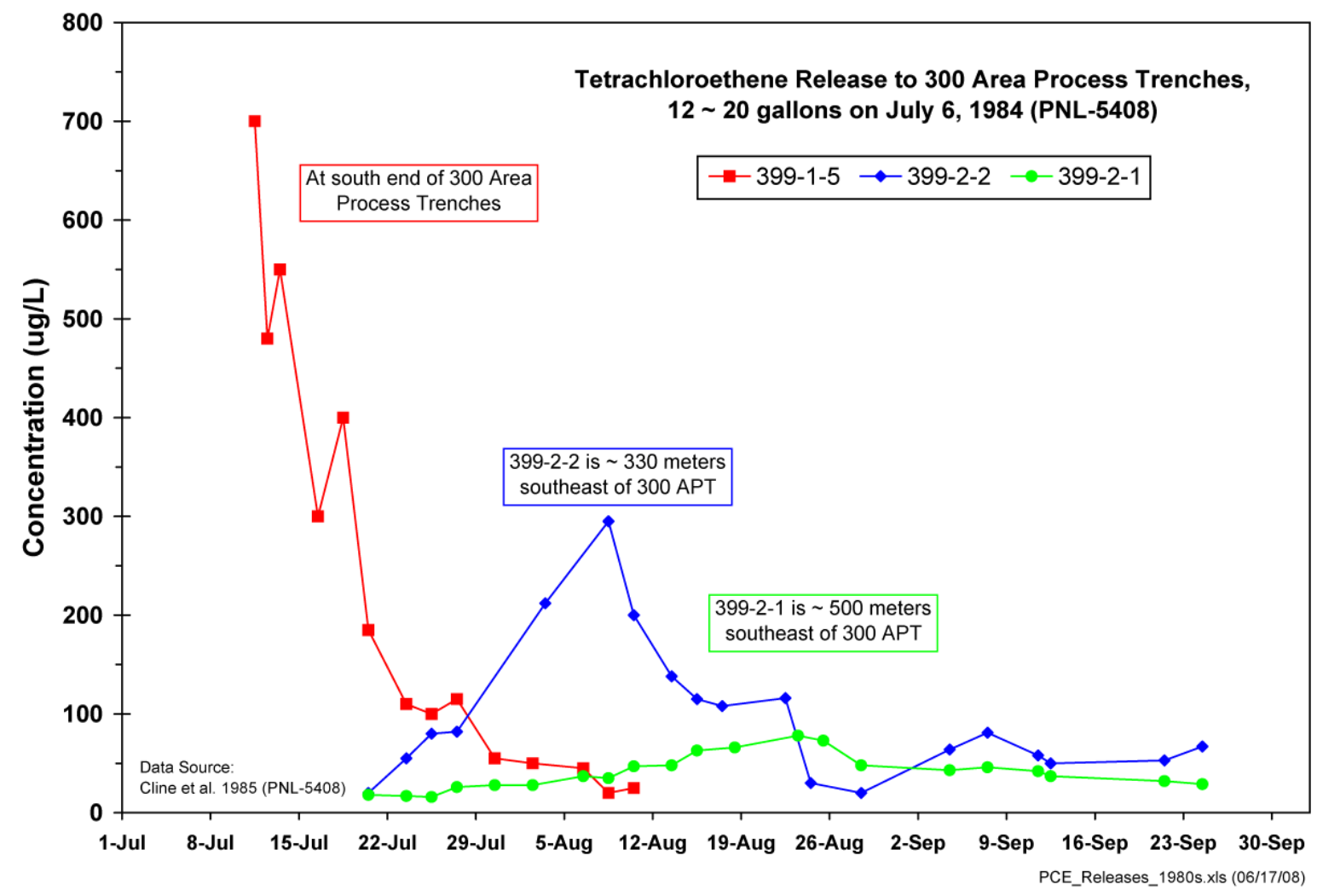

Figure 3.2. Tetrachloroethene in Groundwater from Release on July 6, 1984

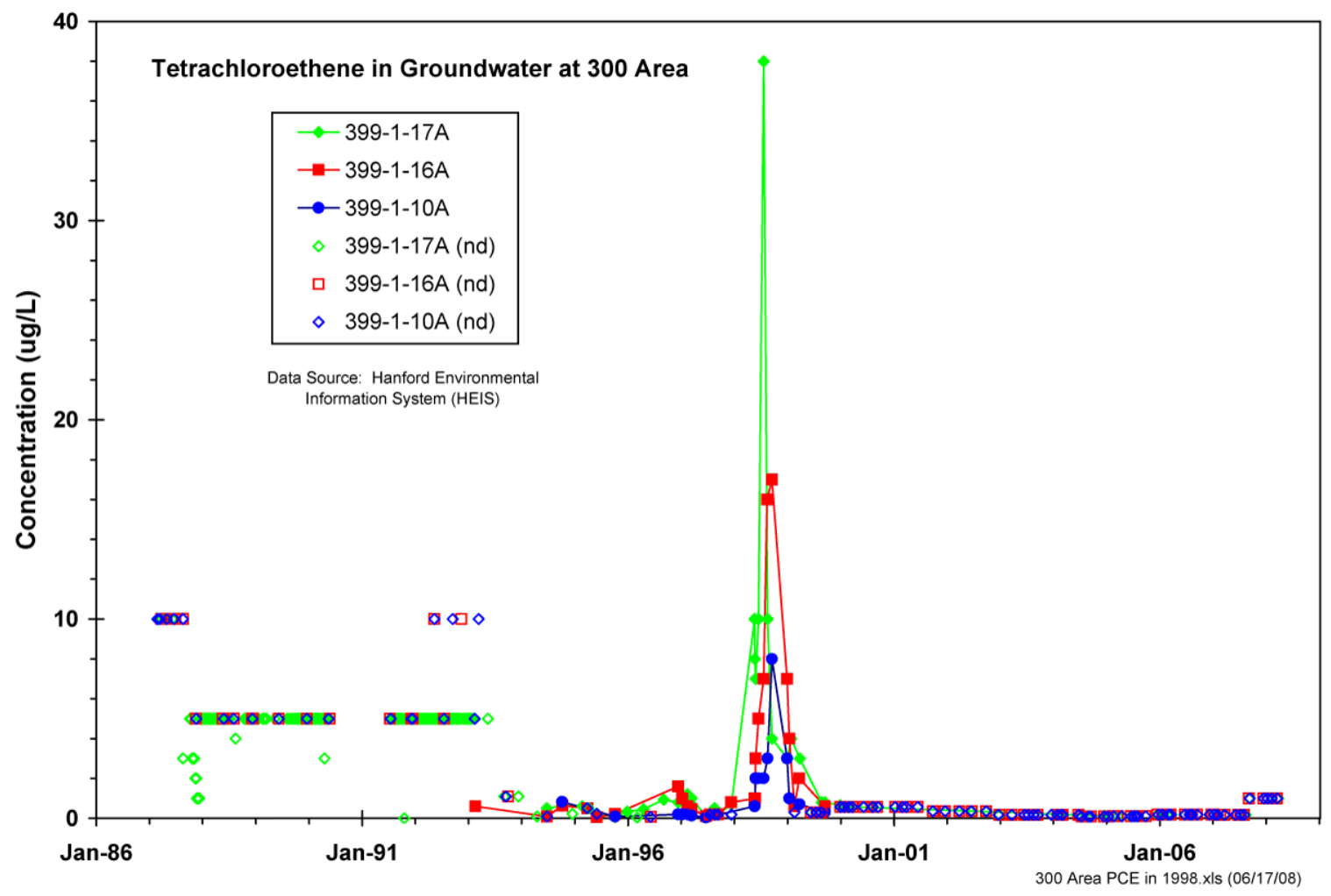

Figure 3.3. Increased Tetrachloroethene Concentrations in 300 Area Groundwater During 1998 
In summary, TCE and PCE were widely used in the 300 Area in degreasing operations associated with fuel fabrication activities and also in the various radiological laboratories. However, no definitive evidence for significant spills or leakage from storage and distribution pipes, other than the accidental spills and events described above, could be found. Disposal of used degreasers apparently was to the process sewer system, which discharged to the North and South process ponds during the peak production years. However, the review of historical information available has shed few clues as to the origin for the elevated trichloroethene concentrations observed in the finer-grained Ringold sediment interval during the LFI and subsequent VOC investigation.

Assuming that significant quantities of TCE and PCE were included in the effluent that went to the North and South process ponds during the peak fuels production years (1950s and 1960s), it is reasonable to also assume that these contaminants were carried downward through the vadose zone and into the aquifer. The volume of effluent that infiltrated through the ponds may have been sufficient to increase the downward hydraulic gradient in the unconfined aquifer, thus driving the contaminants into deeper horizons, including the finer-grained interval. Whether the higher density of the VOCs (compared to other waste effluent constituents) contributed to this downward movement is not known.

Following the end of discharge to the two process ponds in the mid-1970s, the rapid movement of groundwater through the saturated Hanford formation sediment in the upper portion of the unconfined aquifer would have removed evidence for the earlier contamination. However, contaminated groundwater that had come in contact with the deeper finer-grained interval may have migrated slowly into those silty sandy sediments, where it remains today as residual contamination from earlier conditions. The presence of VOC contamination only, without evidence for co-contaminants associated with effluents discharged to the process ponds (e.g., uranium), is perplexing, as it suggests that individual contaminants were dispersed by different transport processes.

Figure 3.4 provides a summary of information used to infer the lateral extent of the TCE contamination (vertical extent is limited to the finer-grained interval of Ringold sediment). The solid green circles on Figure 3.4 show where elevated levels of TCE were observed in groundwater samples from the finer-grained interval during LFI and VOC investigation drilling and at aquifer tube sites. The dashed green circles show where contamination was not observed during drilling. The dashed black line outlines the general area of contamination and is based on observations in wells and assumptions concerning the source for the VOCs, that is, past discharges to the South Process Pond. 


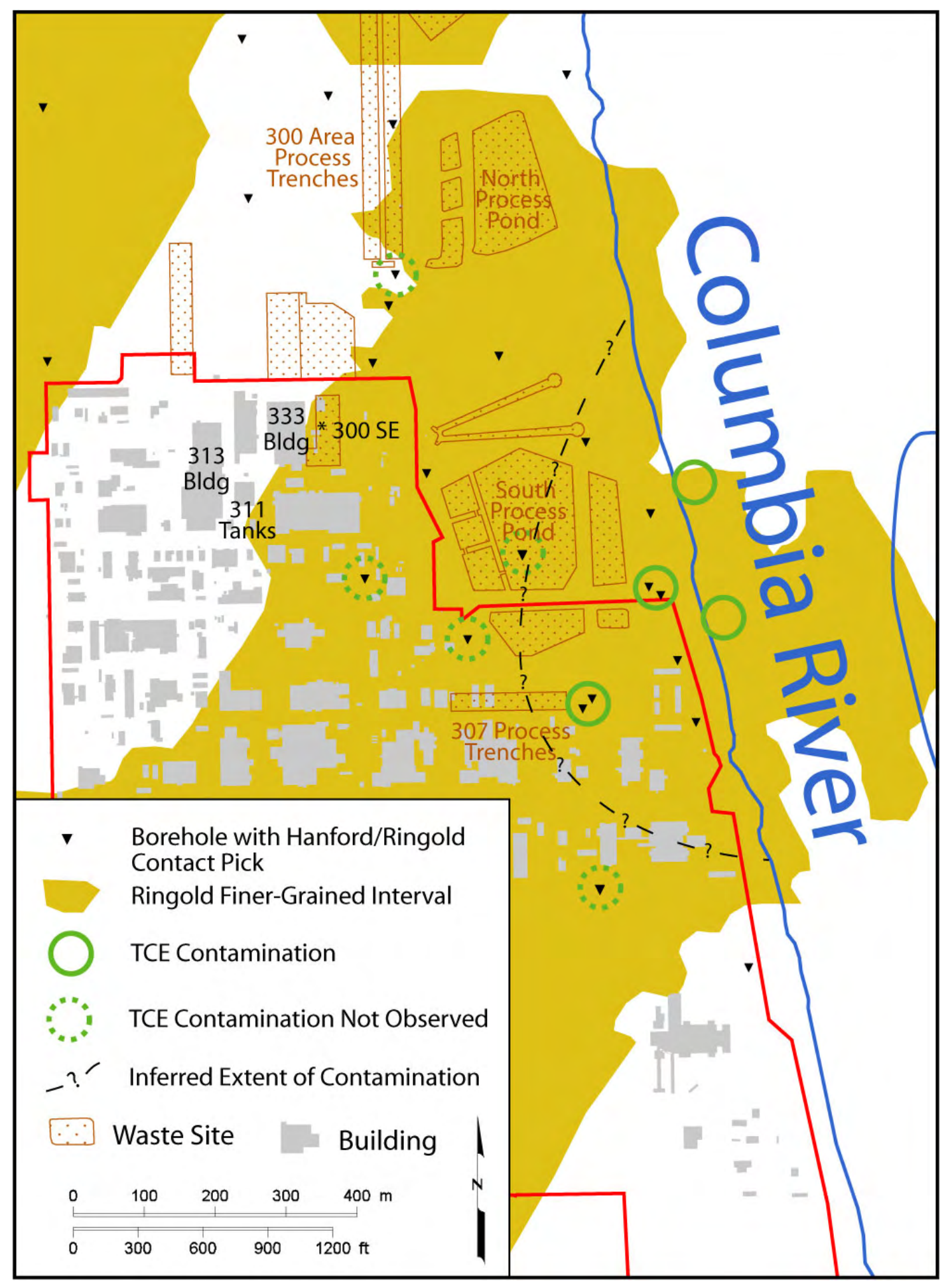

Figure 3.4. Inferred Extent of TCE Contamination in the Finer-Grained Interval of Ringold Formation Unit E. The two circles at the shoreline are aquifer tube sites. 


\subsection{Environmental Pathways Associated with VOC Contamination}

The following sections discuss the potential pathways by which the VOC contamination encountered in groundwater associated with the finer-grained interval of Ringold Formation sediment may lead to potential contact by humans and other biological receptors. The discussion includes identification of potential pathways to such contact and an analysis of the potential magnitude of the exposure.

\subsubsection{Pathways to Exposure}

VOC contamination in the finer-grained interval within the unconfined aquifer beneath the 300 Area could conceivably be dispersed via the following environmental pathways:

- Aquifer Pathways

- lateral migration with groundwater flow through the finer-grained Ringold interval to areas in the Columbia River substrate where the channel has incised the contaminated interval:

$\circ \quad$ uptake by aquatic plants and animals (benthic habitat)

$\circ$ human contact (dermal exposure during swimming)

- vertical dispersion out and upward from the finer-grained interval to the more transmissive overlying Hanford formation sediment:

$\circ$ mixes with VOC contamination of lower concentration from other sources, before migrating to the river environment

- uptake by aquatic plants and animals via riverbank springs (riparian habitat)

- groundwater withdrawal for water supply purposes:

$\circ$ human contact (ingestion as drinking water)

○ uptake by terrestrial plants and animals (via irrigation)

- Surface Water Pathways

- Surface water is an extension of the unconfined aquifer pathway:

- degradation of Columbia River water quality

$\circ$ uptake by aquatic organisms in the free-flowing stream

- Atmospheric Pathway:

$\circ$ The atmospheric pathway could be considered a secondary pathway if contaminated groundwater or river water were to be used for irrigation.

○ Human and ecological receptors (via inhalation)

- Soil Pathway

- VOC contamination has been detected only in subsurface reservoirs involving groundwater, so this pathway is not considered a primary pathway for migration.

\subsubsection{Aquifer Pathway Evaluation}

The unconfined aquifer is the principal pathway by which VOC contamination found in the finergrained interval of the Ringold Formation can be transported via groundwater flow to exposure locations. Therefore, it is discussed in more detail in the following paragraphs. Contamination present in the unconfined aquifer could conceivably result in human or environmental exposure via natural migration 
through the groundwater system to exposure locations at riverbank springs or within the river channel. Withdrawals of groundwater from wells could also conceivably result in human and ecological receptor exposures to contamination.

VOC contamination found in the finer-grained interval is dissolved in groundwater and is expected to disperse from locations where the contamination was initially introduced via movement of groundwater in that interval. Two possible migration pathways are 1) lateral migration through the finer-grained interval to the area where the unit outcrops in the river bottom and 2) vertical migration upward (i.e., slow release out of the finer-grained interval) into the overlying Hanford formation gravels, where it would mix and migrate with groundwater in that hydrologic unit. Note that groundwater in the Hanford formation hydrologic unit already is contaminated with VOCs but at concentrations typically lower than the drinking water standards. The origin for this contamination is previous disposal at 300 Area waste sites and migration of contaminated groundwater into the 300 Area from source locations to the southwest (Peterson and Lindberg 2008, pp. 2.12-7-2.12-9).

Groundwater flowing through the Hanford formation reaches the Columbia River and discharges through the riverbed and, to a lesser degree, via riverbank springs during periods of low river stage. Monitoring riverbank spring water for VOCs is conducted as part of the Surface Environmental Surveillance Project. When detected, concentrations are very low and below relevant standards (Patton et al. 2002, p. 4.11; Patton 2007, p. 10.52). Analysis of river water has not detected the presence of VOCs attributable to discharge of contaminated groundwater through the riverbed (Patton 2007, p. 10.39). Also, analysis of river water collected as part of this investigation did not reveal VOCs at water depths within approximately one meter or less of the river bottom. Detection of VOCs in the free-flowing stream of the Columbia River in the Hanford Reach would not be expected, even if there were clear indication of contaminated groundwater discharge through the riverbed, because of the volatility of VOCs in the rapidly flowing river water and therefore a short residence time that is measured in days or less.

Withdrawal of groundwater for human consumption is a possible future scenario and could be considered a completed exposure pathway. Alternative withdrawal configurations considered in this evaluation include 1) withdrawal only from the finer-grained interval in the Ringold Formation (worstcase scenario, but also an unlikely future scenario for multiple reasons) and 2) withdrawal from a well that is screened across the full vertical extent of the Hanford formation hydrologic unit.

In the first case, the well screen vertical extent is limited to the thickness of the finer-grained sandy interval and is isolated from the more transmissive gravelly intervals above and below. Using hydraulic conductivities measured during LFI and VOC investigation drilling, investigators calculated that a standard water well of 8-inch diameter and a 10-foot thick production zone could sustain a constant pumping rate of 1 gallon per minute (1,440 gallons per day) for about 1,000 days before the zone was pumped dry. For comparison, the city of Richland suggests that typical household usage of water is about 250 gallons per day (http://www.ci.richland.wa.us “Living” and “Environmental Education”). However, the city also notes that water use increases by an additional 500 to 1,000 gallons per day on average during the summer and, depending on the size of the property, up to 3,000 gallons per day for peak usage. So a well drawing from this unit might serve the needs of only several households and for only several years, and would also have to address the VOC contamination in the water. 
A more realistic scenario for future groundwater use (i.e., post-institutional controls) would involve completing a well in the more productive Hanford formation, where the hydraulic conductivity is sufficiently high so as to yield adequate water for household and irrigation purposes. ${ }^{1}$ VOC exposure for this scenario would result from VOCs being drawn out of the finer-grained interval in the underlying Ringold Formation and migrating upward into the Hanford formation. Because of the high permeability contrast, only a small portion of the water drawn into the well during pumping would have the finegrained Ringold layer as its source. Any TCE from the fine-grained layer would be mixed with the less contaminated Hanford formation groundwater during pumping. Because of this dilution, even if TCE drawn from the fine-grained Ringold material were able to sustain the maximum TCE concentrations detected in Hanford formation groundwater (i.e., $19 \mu \mathrm{g} / \mathrm{L}$ during the VOC investigation drilling) in a 5-foot thick sampling interval just above the Ringold finer-grained interval, the water produced from a supply well completed across the entire Hanford formation (approximately 30 feet in thickness) would likely contain TCE at concentrations below the drinking water standard of $5 \mu \mathrm{g} / \mathrm{L}$.

\subsection{VOC Investigation and cis-DCE at Well 399-1-16B}

Elevated concentrations of cis-1,2-dichloroethene (DCE) have been observed at well 399-1-16B, located to the north of the VOC investigation region (see Figure 1.2) for many years. The position of this well is shown also on the cross sections in Figures 2.4 and 2.11. A concentration trend chart for the well is shown in Figure 3.5. While little documentation exists to explain the occurrence and possible sources for DCE at this well, it is generally assumed to be a localized contaminant plume, with disposal at the former North Process Pond and/or 300 Area Process Trenches being the likely source waste sites (Lindberg and Chou 2001; Peterson et al. 2005). The accidental releases of PCE to the 300 Area Process Trenches in 1982 and 1984 (described in Section 3.1) may be implicated, but the connection is speculative at this time.

DCE is a likely degradation product of TCE and/or PCE, which both were used extensively in the fuels fabrication process and were likely components of waste effluents disposed to infiltration trenches and ponds. Under suitable environmental conditions in the aquifer, TCE and PCE may degrade via anaerobic dechlorination processes to form DCE, which may or may not degrade further, depending on the presence of certain bacteria (see discussion in Section 1.2). Conditions at the base of the unconfined aquifer may be conducive to a scenario whereby TCE (or PCE) was released to the ground in sufficient quantity to drive it downward into the lower portion of the unconfined aquifer and then to degrade to DCE.

A determination of whether a causal relationship between the occurrence of DCE at well 399-1-16B and the elevated concentrations of TCE in the finer-grained interval of the Ringold Formation in the area to the south cannot be established conclusively at this time. The occurrence of DCE at well 399-1-16B was not part of the VOC investigation that is the subject of this report. To further investigate the origin for DCE at that well would likely require additional field investigation because little evidence for its origin has yet been uncovered in documents describing historical operations and events.

\footnotetext{
${ }^{1}$ A water supply well (399-4-12) has been in use in the 300 Area since 1982 to provide water for aquariums in the PNNL Life Sciences Building. That well is screened across approximately one-half of the saturated Hanford formation gravels hydrologic unit (see Figure 2.11) and is capable of producing hundreds of gallons per minute.
} 


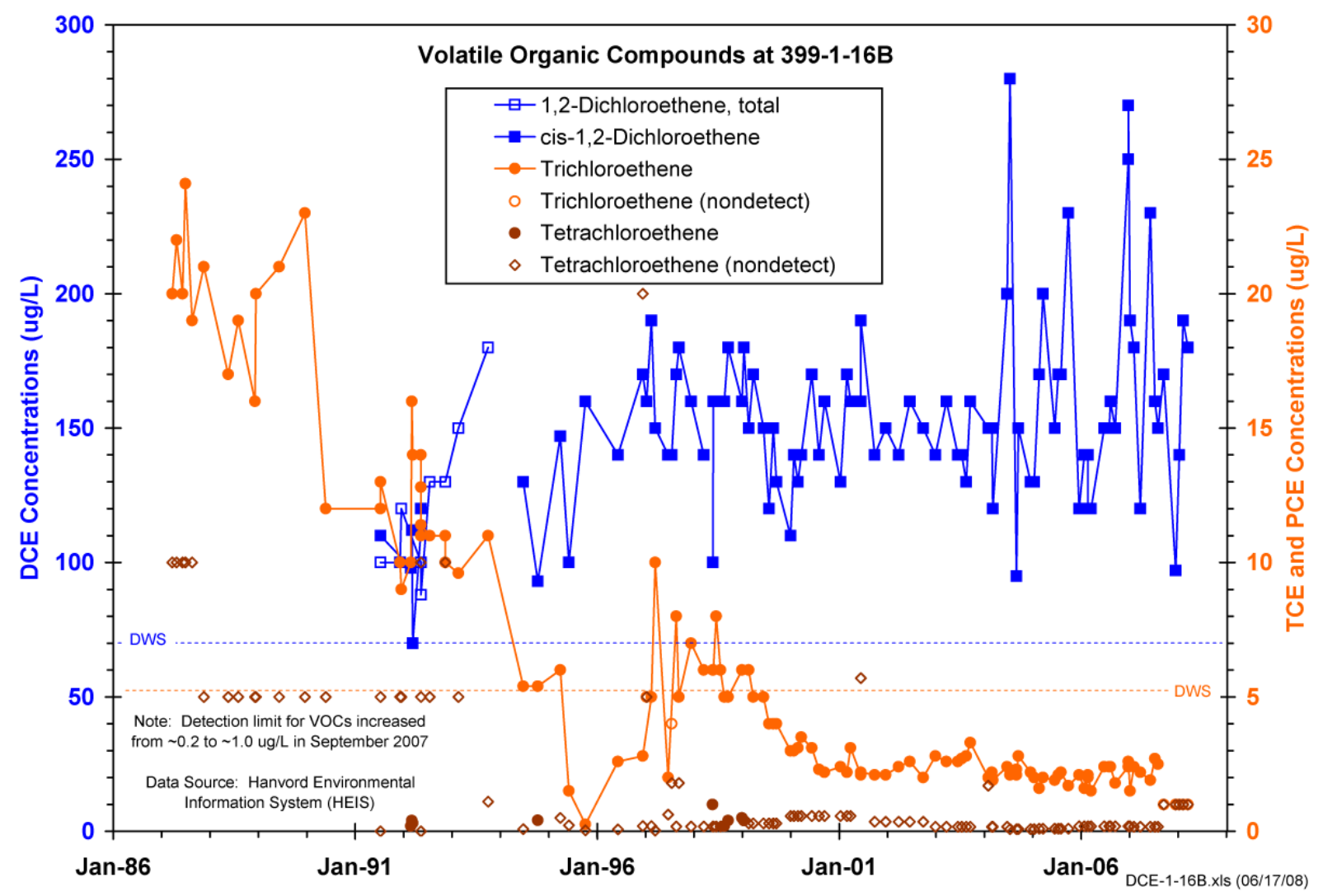

Figure 3.5. Cis-1,2-Dichloroethene Concentrations in Groundwater at Well 399-1-16B (Lower Portion of Unconfined Aquifer) 



\subsection{Conclusions}

New information regarding the nature and extent of contamination by volatile organic compounds has been obtained by drilling additional characterization boreholes. The new information provides answers to several questions that remained following the initial discovery during an earlier limited field investigation associated with uranium contamination. Principle conclusions from information obtained during the recent and earlier investigations follow:

- Elevated concentrations of volatile organic compounds are present in groundwater at several locations in the east-central region of the 300 Area in an interval of finer-grained sediment within the unconfined aquifer. The finer-grained sediment containing the contamination is much less permeable than the overlying highly permeable gravelly sediment, in which the concentrations of volatile organic compounds are lower than the drinking water standards. This contamination has not been encountered at depths greater than the finer-grained sediment at the locations investigated.

- The anomalous occurrences of volatile organic compounds appears to be limited to an area east and south of the former South Process Pond and east of the former 307 Process Trenches, although the areal extent of the finer-grained interval of sediment is more extensive and covers a large portion of the area beneath the 300 Area.

- The primary volatile organic compound of concern is trichloroethene, which was used extensively as a degreasing agent during fuel fabrication operations and is known to have been discharged to the principal liquid waste disposal facilities (e.g., process ponds). Much lower concentrations were observed for tetrachloroethene, which also was used as a degreaser, and cis-1,2-dichloroethene, a degradation product of trichloroethene and tetrachloroethene. Vinyl chloride, a degradation product under some environmental conditions, is not detected in the groundwater beneath the 300 Area.

- The exact source(s) and timing of releases for the volatile organic compounds observed during the investigation are not known. However, based on the history of operations, it can be inferred that those operations included the release of relatively large quantities of trichloroethene and tetrachloroethene to liquid waste disposal sites, with the North and South process ponds likely candidates during the 1950s and 1960s. These releases may have moved downward in sufficient quantities to contaminate not only the upper part of the unconfined aquifer but also sediment in the finer-grained interval that typically appears deeper in the aquifer. To date, no evidence for a dense, nonaqueous phase liquid has been discovered. During post-operations years, contamination has been flushed from the upper part of the unconfined aquifer because of the high permeability of the formation.

- Exposure pathways for the trichloroethene observed in the finer-grained interval of Ringold Formation sediment include 1) movement toward the Columbia River, where the channel incises to depths with elevations similar to those projected for the finer-grained interval; 2) dispersion out of the finer-grained sediment and into the more permeable overlying Hanford formation gravels hydrologic unit, which is exposed at the riverbed; and 3) withdrawal of contaminated groundwater from wells that might penetrate the finer-grained unit. However, any future water supply well would not be constructed to penetrate that unit (because of its low groundwater yield), and any contamination that might inadvertently be pulled from the unit would be rapidly mixed with the cleaner groundwater from the overlying saturated Hanford formation gravels 



\subsection{References Cited}

ANL. 2007. "Radiological and Chemical Fact Sheets to Support Health Risk Analyses for Contaminated Areas.” Environmental Science Division, Argonne National Laboratory, Argonne, Illinois. Available at http://www.ead.anl.gov/pub/doc/ANL_ContaminantFactSheets_All_070418.pdf (July 2008).

Bjornstad BN. 2004. Sampling and Hydrogeology of the Vadose Zone Beneath the 300 Area Process Ponds. PNNL-14834. Pacific Northwest National Laboratory, Richland, Washington.

Cline CS, JT Rieger, JR Raymond, and PA Eddy. 1985. Ground-Water Monitoring at the Hanford Site, January-December 1984. PNL-5408, Pacific Northwest Laboratory, Richland, Washington.

Clukey HV. 1954. 300 Area Radioactive Liquid Waste Streams Disposal. HW-32762, General Electric Company, Richland, Washington.

Cohen RM and JW Mercer. 1993. DNAPL Site Evaluation. CRC Press, Inc., Boca Raton, Florida.

Deford DH, RW Carpenter, and MW Einan. 1994. 300-FF-2 Operable Unit Technical Baseline Report. BHI-00012, Rev. 00, Bechtel Hanford, Inc., Richland, Washington.

Dennison DI, DR Sherwood, and JS Young. 1989. Status Report on Remedial Investigation of the 300 Area Process Ponds. PNL-6442, Pacific Northwest Laboratory, Richland, Washington.

DOE-RL. 2002. Standardized Stratigraphic Nomenclature for Post-Ringold-Formation Sediments Within the Central Pasco Basin. DOE/RL-2002-39, Rev. 0, U.S. Department of Energy, Richland Operations Office, Richland, Washington.

Fritz BG, RD Mackley, NP Kohn, GW Patton, TJ Gilmore, DP Mendoza, D McFarland, AL Bunn, and EV Arntzen. 2007. Investigation of the Hyporheic Zone at the 300 Area, Hanford Site. PNNL-16805, Pacific Northwest National Laboratory, Richland, Washington.

Gaylord DR and EP Poeter. 1991. Geology and Hydrology of the 300 Area and Vicinity, Hanford Site, South-Central Washington. WHC-EP-0500, Westinghouse Hanford Company, Richland, Washington.

Gerber MS. 1992. Past Practices Technical Characterization Study - 300 Area -Hanford Site. WHC-MR-0388, Westinghouse Hanford Company, Richland, Washington.

Horner JA. 2008. Borehole Summary Report for 300-FF-5 Operable Unit TCE Characterization Monitoring Wells C5575, C5706, C5707, and C5708. SGW-36424, Rev. 0, Fluor Hanford, Inc., Richland, Washington.

Kooiker CA, RE Peterson, SM Smith, and BA Williams. 2007. Sampling and Analysis Instructions for TCE Characterization, 300-FF-5 Operable Unit, Fiscal Year 2007. SGW-32607, Rev. 0, Fluor Hanford, Inc., Richland, Washington.

Lindberg JW and FW Bond. 1979. Geohydrology and Groundwater Quality Beneath the 300 Area, Hanford Site, Washington. PNL-2949, Pacific Northwest National Laboratory, Richland, Washington. 
Lindberg JW and CJ Chou. 2001. 300 Area Process Trenches Groundwater Monitoring Plan. PNNL-13645, Pacific Northwest National Laboratory, Richland, Washington.

Lindsey KA. 1995. Miocene- to Pliocene-Aged Suprabasalt Sediments of the Hanford Site, SouthCentral Washington. BHI-00184, Rev. 00, Bechtel Hanford, Inc., Richland, Washington.

Loe JW. 1967. An Investigation of Effluent Control Standards and Practices (300 Area). DUN-3155, Douglas United Nuclear, Inc., Richland, Washington.

Luttrell SP and WD Webber. 2008. “1100-EM-1 Operable Unit.” Chapter 2.13 in Hanford Site Groundwater Monitoring for Fiscal Year 2007, MJ Hartman and WD Webber (eds.). DOE/RL-2008-01, Rev. 0, U.S. Department of Energy, Richland Operations Office, Richland, Washington.

Mackley RD and BG Fritz. 2007. Characterizing the Hydrogeology of the Hyporheic Zone Along the 300 Area of the Hanford Site, Washington. PNNL-SA-56035, presented to the 6th Washington Hydrogeology Symposium, May 1-3, 2007, Tacoma, Washington.

Newcomb RC, JR Strand, and FJ Frank. 1972. Geology and Ground-Water Characteristics of the Hanford Reservation of the U.S. Atomic Energy Commission, Washington. Geological Survey Professional Paper 717, U.S. Geological Survey, Reston, Virginia. Available at http://pubs.er.usgs.gov/djvu/PP/pp 717.djvu (July 2008).

Newcomer DR. 2008. 300 Area VOC Program Slug Test Characterization Results for Selected Test/Depth Intervals for Wells 399-2-5, 399-3-22, and 399-4-14. PNNL-17439, Pacific Northwest National Laboratory, Richland, Washington.

NAD83. 1991. North American Datum of 1983. National Geodetic Survey, Federal Geodetic Control Committee, Silver Spring, Maryland, as revised.

NAVD88. 1988. North American Vertical Datum of 1988. National Geodetic Survey, Federal Geodetic Control Committee, Silver Spring, Maryland.

Patton GW. 2007. “10.5 Columbia River Shoreline Springs Monitoring.” In: Hanford Site Environmental Report for Calendar Year 2006. TM Poston, RW Hanf, JP Duncan, and RL Dirkes (eds). PNNL-16623, Pacific Northwest National Laboratory, Richland, Washington.

Patton GW, SP Van Verst, BL Tiller, EJ Antonio, and TM Poston. 2002. Survey of Radiological and Chemical Contaminants in the Near-Shore Environment at the Hanford Site 300 Area. PNNL-13692, Pacific Northwest National Laboratory, Richland, Washington.

Peterson RE and JW Lindberg. 2008. “300-FF-5 Operable Unit.” Chapter 2.12 in Hanford Site Groundwater Monitoring for Fiscal Year 2007, MJ Hartman and WD Webber (eds.). DOE/RL-2008-01, Rev. 0, U.S. Department of Energy, Richland Operations Office, Richland, Washington.

Peterson RE, EJ Freeman, PD Thorne, MD Williams, JL Lindberg, CJ Murray, MJ Truex, SB Yabusaki, JP McDonald, VR Vermeul, and JM Zachara. 2005. Contaminants of Potential Concern in the 300-FF-5 Operable Unit: Expanded Annual Groundwater Report for FY 2004. PNNL-15127, Pacific Northwest National Laboratory, Richland, Washington. 
Peterson, RE, RJ Serne, PD Thorne, MD Williams, and ML Rockhold. 2008. Uranium Contamination in the Subsurface Beneath the 300 Area, Hanford Site, Washington. PNNL-17034, Pacific Northwest National Laboratory, Richland, Washington.

Reidel SP, KA Lindsey, and KR Fecht. 1992. Field Trip Guide to the Hanford Site. WHC-MR-0391, Westinghouse Hanford Company, Richland, Washington.

Swanson LC, GG Kelty, KA Lindsey, KR Simpson, RK Price, and SD Consort. 1992. Phase I Hydrogeologic Summary of the 300-FF-5 Operable Unit, 300 Area. WHC-SD-EN-TI-052, Rev. 0, Westinghouse Hanford Company, Richland, Washington.

Thorne PD, MA Chamness, FA Spane, VR Vermeul, and WD Webber. 1993. Three-Dimensional Conceptual Model for the Hanford Site Unconfined Aquifer System: FY 1993 Status Report. PNL-8971, Pacific Northwest Laboratory, Richland, Washington.

Thorne PD. 2008. "Hydrogeologic Framework." Section 4 in Uranium Contamination in the Subsurface Beneath the 300 Area, Hanford Site, Washington, RE Peterson, ML Rockhold, RJ Serne, PD Thorne, and MD Williams. PNNL-17034, Pacific Northwest National Laboratory, Richland, Washington.

Williams BA, BN Bjornstad, R Schalla, and WD Webber. 2000. Revised Hydrogeology for the Suprabasalt Aquifer System, 200-East Area and Vicinity, Hanford Site, Washington. PNNL-12261, Pacific Northwest National Laboratory, Richland, Washington.

Williams BA, CF Brown, W Um, MJ Nimmons, RE Peterson, BN Bjornstad, DC Lanigan, RJ Serne, FA Spane, and ML Rockhold. 2007. Limited Field Investigation Report for Uranium Contamination in the 300 Area, 300-FF-5 Operable Unit, Hanford Site, Washington. PNNL-16435, Pacific Northwest National Laboratory, Richland, Washington.

Young JS and JS Fruchter. 1991. Addendum to Data Compilation Task Report for the Source Investigation of the 300-FF-1 Operable Unit Phase I Remedial Investigations. EMO-1026, U.S. Department of Energy, Richland Operations Office, Richland, Washington. 



\section{Appendix}

Composite Borehole Logs for VOC Investigation and Limited Field Investigation Drilling 

399-3-21 (C5575)
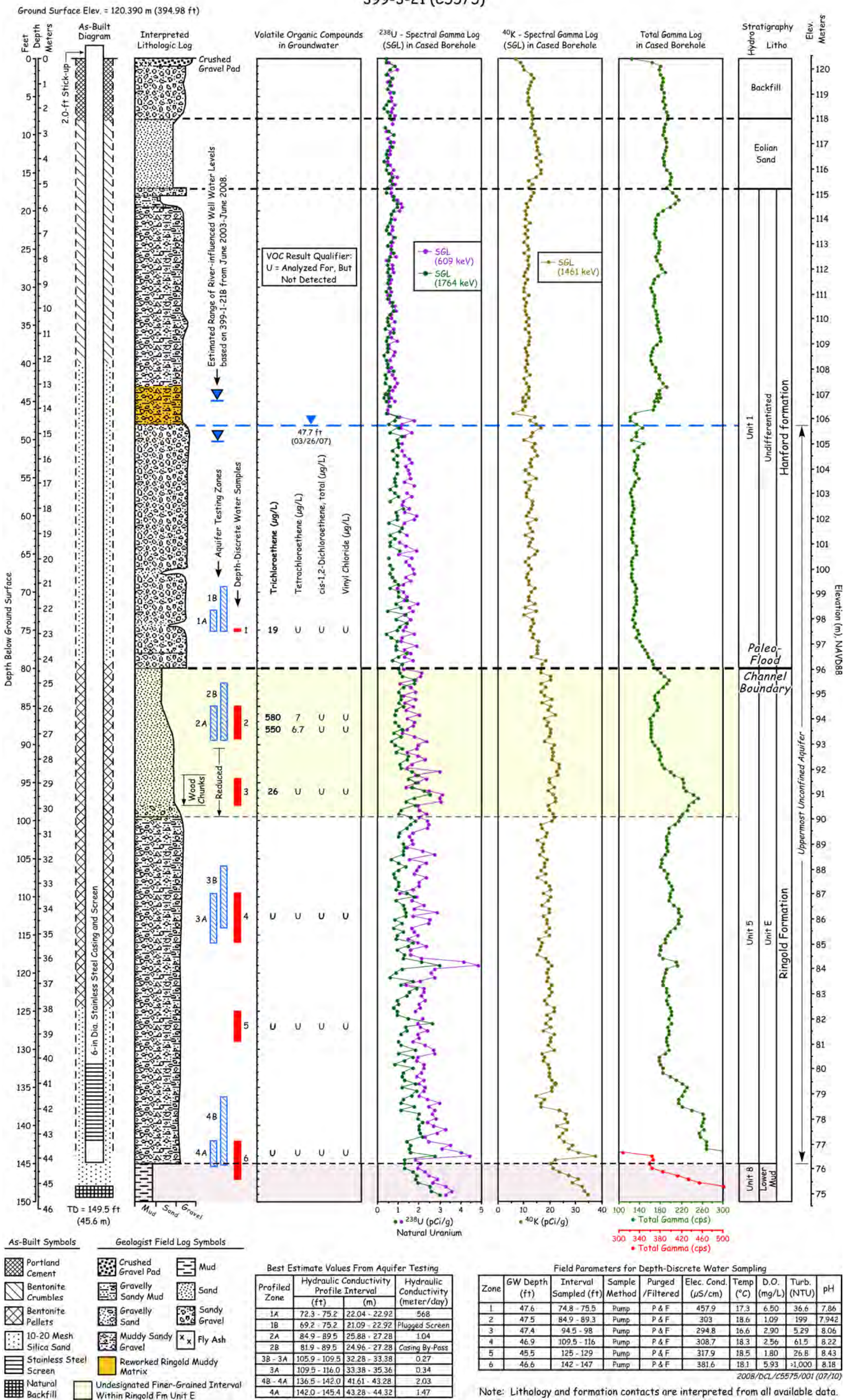

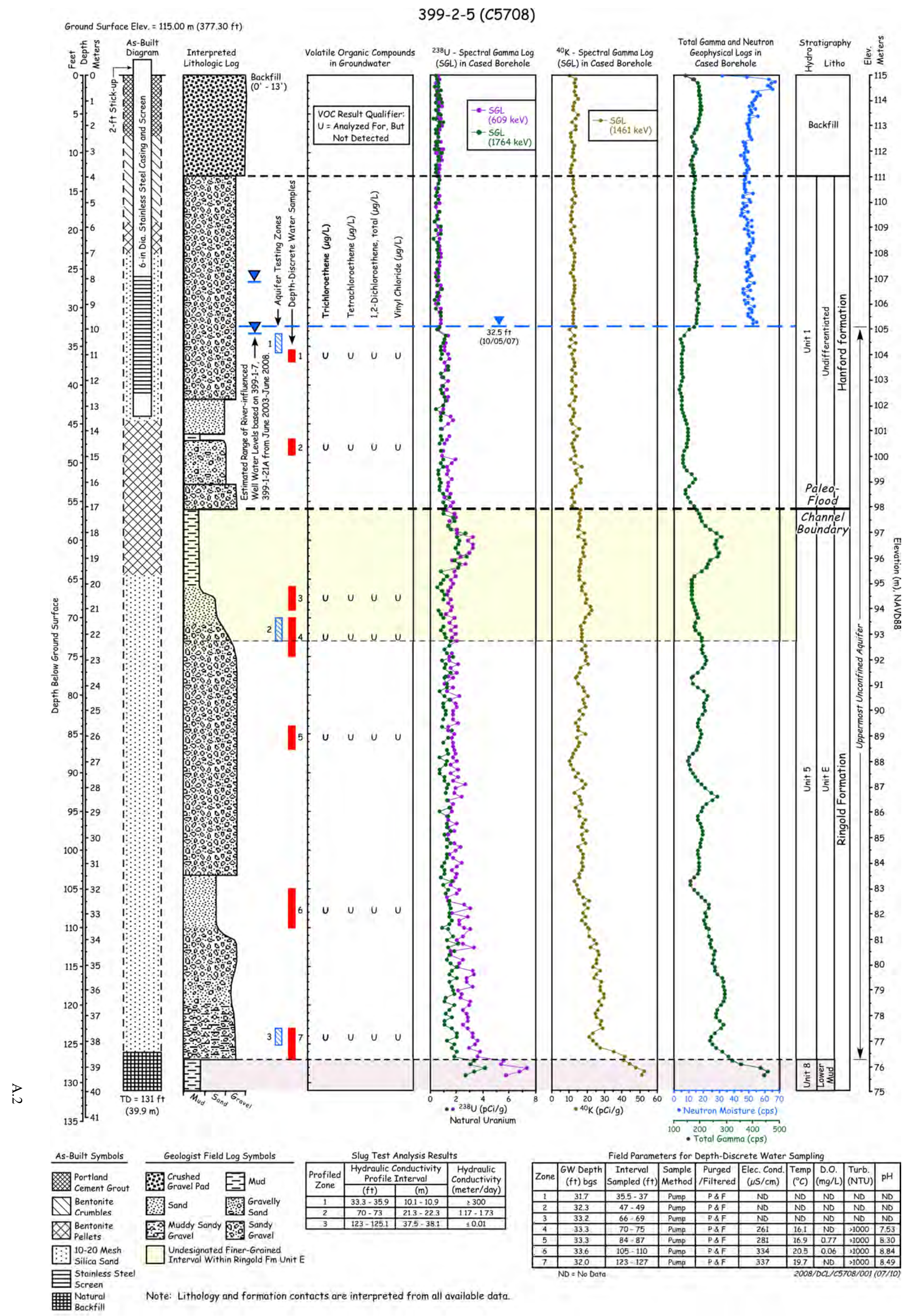

Figure A.2. Composite Borehole Log for 399-2-5 (C5708) 


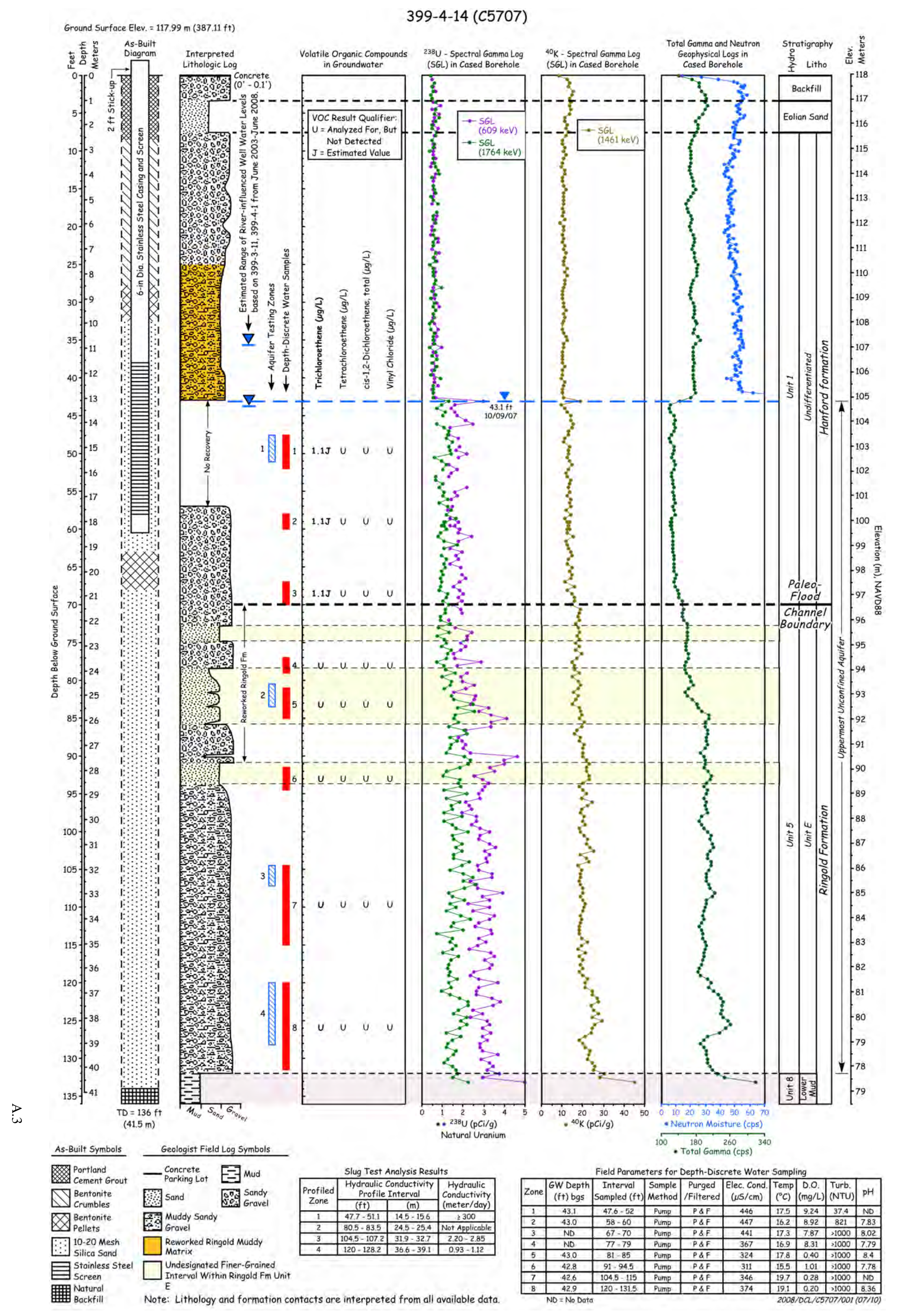

Figure A.3. Composite Borehole Log for 399-4-14 (C5707) 
$399-3-22(C 5706)$
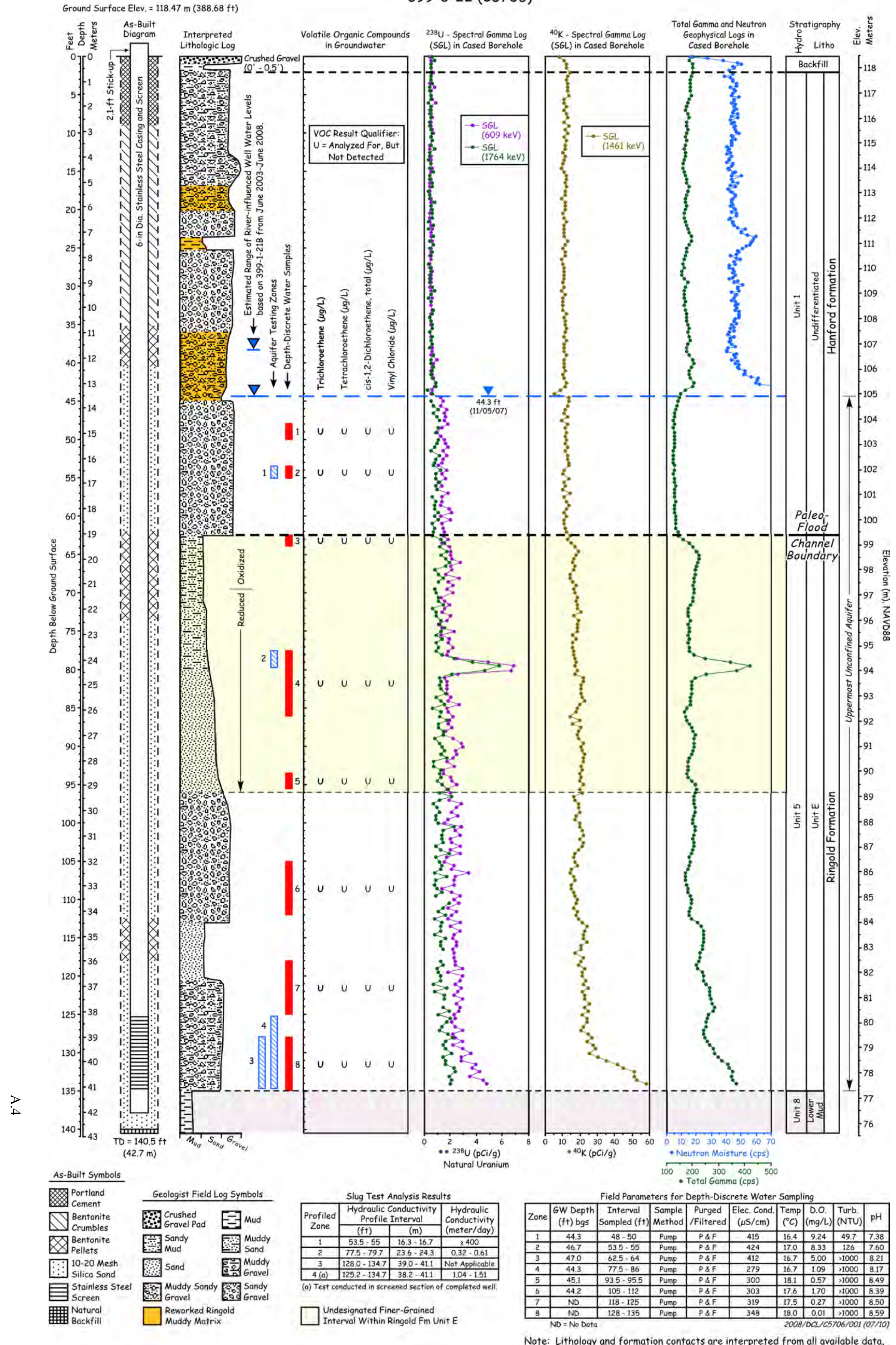

Note: Lithology and formation contacts are interpreted from all available data.

Figure A.4. Composite Borehole Log for 399-3-22 (C5706) 


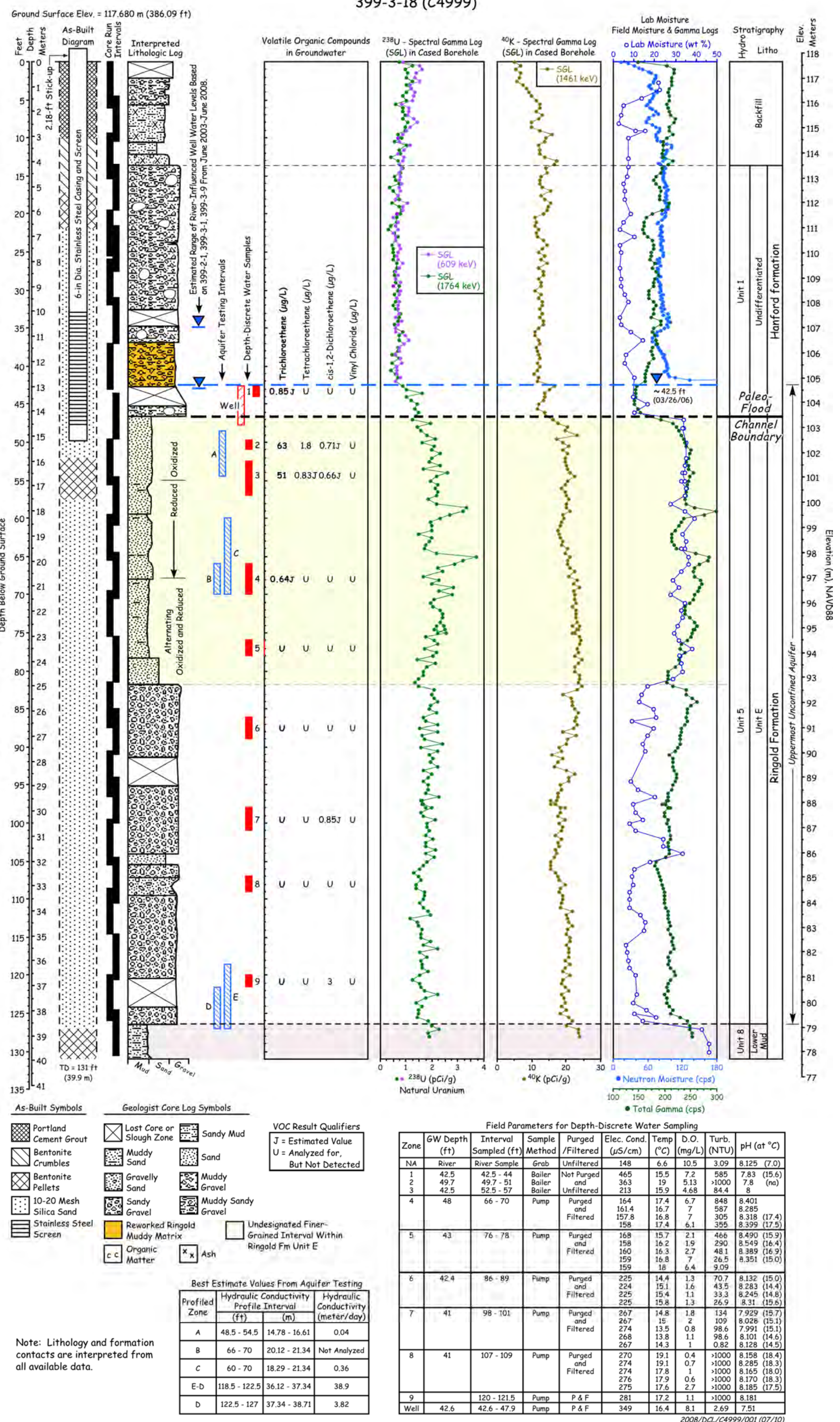



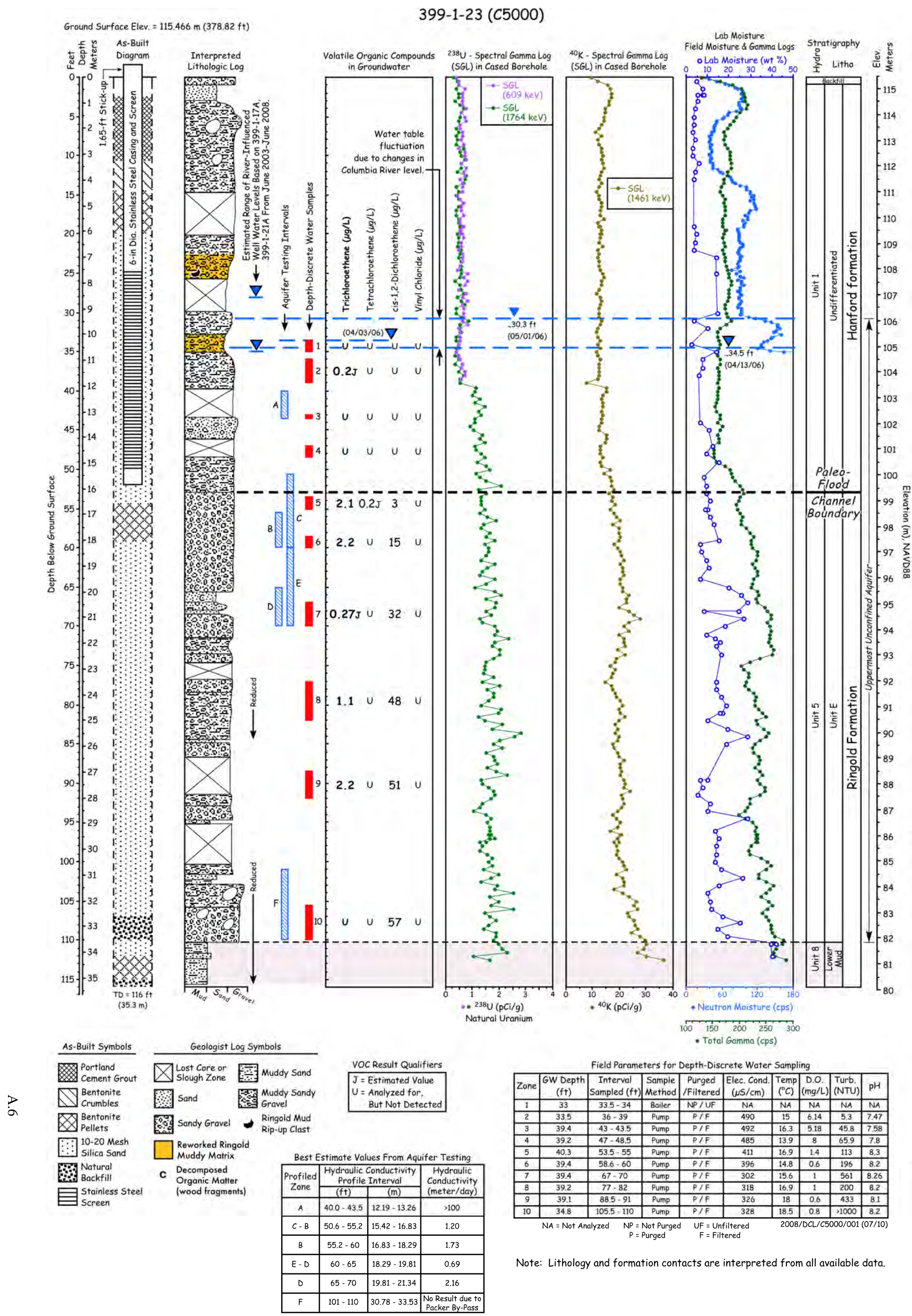

Note: Lithology and formation contacts are interpreted from all available data.

Figure A.6. Composite Borehole Log for LFI Borehole 399-1-23 (C5000) 


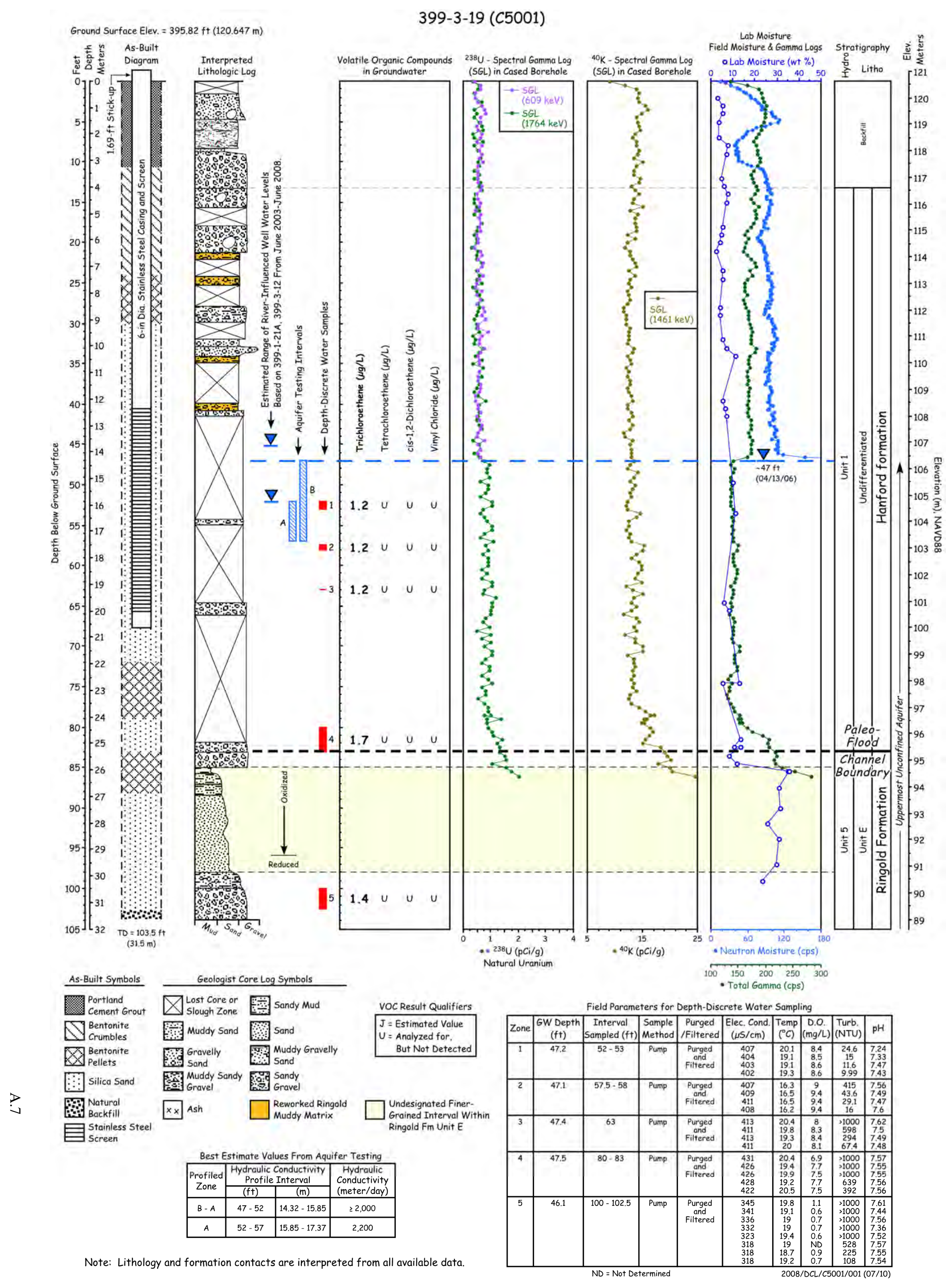

Figure A.7. Composite Borehole Log for LFI Borehole 399-3-19 (C5001) 


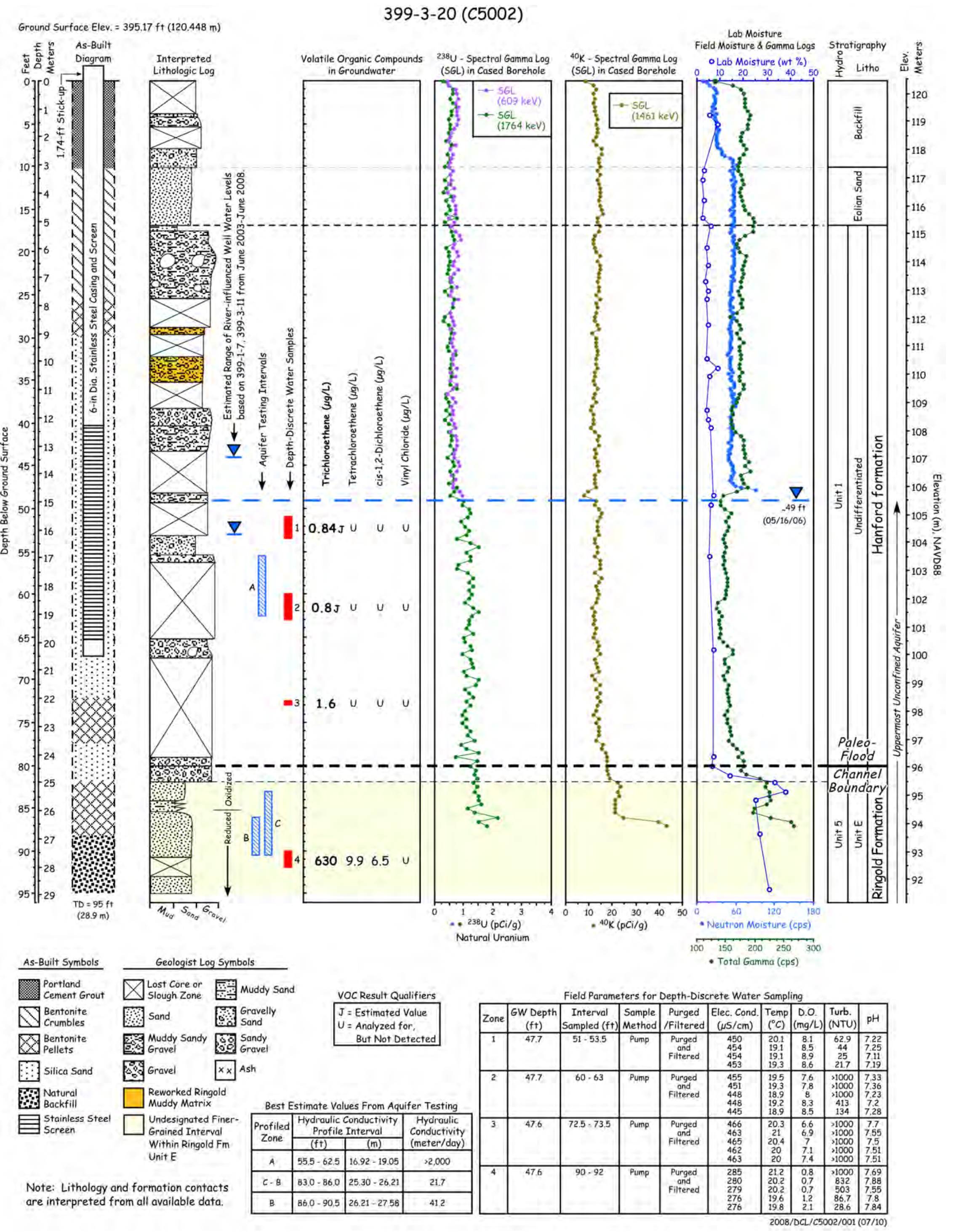

Figure A.8. Composite Borehole Log for LFI Borehole 399-3-20 (C5002) 


\section{Distribution}

No. of

Copies

7 DOE-Richland Operations Office

KM Thompson (5)

JP Sands

J Zeisloft

EPA-Richland

AL Boyd

B1-46

Ecology

J Price

8 Fluor Hanford, Inc.

JV Borghese (5)

MJ Hartman

JW Lindberg

BA Williams

A6-38

A3-04

A3-04
No. of

Copies

2 Washington Closure Hanford, Inc.

18 Pacific Northwest National Laboratory

BN Bjornstad

K6-81

CF Brown

P7-22

JS Fruchter

K6-96

JA Horner

K6-96

DC Lanigan

K6-75

MJ Nimmons

K6-96

RE Peterson (5)

K6-75

RJ Serne

P7-22

RM Smith

K6-96

PD Thorne

K6-96

MJ Truex

K6-96

MD Williams

K6-96

Hanford Technical Library (2) 


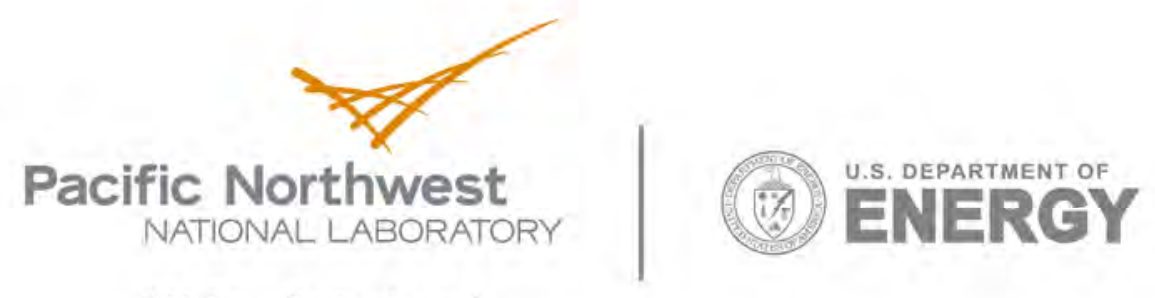

902 Battelle Boulevard

P.O. Box 999

Richland, WA 99352

1-888-375-PNNL (7665)

www.pnl.gov 